COMMUNICATIONS IN

ANALYSIS AND GEOMETRY

Volume 9, Number 2, 281-339, 2001

\title{
Infinitely Spatially Complex Solutions of PDE and Their Homotopy Complexity
}

\author{
V. Afraimovich, A. Babin, and S.-N. ChOW
}

\section{Introduction.}

We consider parabolic two-component systems of the form

$$
\partial_{t} u=\partial_{x}^{2} u-F^{\prime}(u)
$$

on the whole real axis $-\infty<x<\infty$ where $u=\left(u_{1}, u_{2}\right) \in \mathbf{R}^{2}, F(u)$ is a given smooth non-negative potential which has the gradient $F^{\prime}(u)$ which is Lipschitzean in $u$. We consider solutions bounded for all $x, t$. Such solutions include in particular $x$-periodic solutions.

Our main goal is to describe domains in the function space which are invariant with respect to this equation, and which correspond to stable, spatially chaotic patterns. Existence of such domains may model persistence of spatially chaotic patterns in many natural phenomena. It turns out that the spatial behavior can be very complex, and the complexity can be described in terms of appropriate algebraic-topological notions. In the scalar case when $u=u_{1}$, constant solutions $u=m$, where $m=$ const satisfies the equation $F^{\prime}(m)=0$, play an important role. Solutions $m_{i}$ of this equation divide the real line $\mathbf{R}^{1}$ into a number of intervals $\Omega_{i}=\left\{u: m_{i}<u<m_{i+1}\right\}$. If initial data $u(x, 0)$ of (1.1) take value in only one of these intervals, then it is well known that the Maximum Principle implies that the values $u(x, t)$ stay in this interval for all $t \geq 0$ (see [13]). Hence, a geometrical partition of $\mathbf{R}^{1}$ explicitly defines a number of invariant domains in the function space of initial data of (1.1). In the case of the two - component system we consider here, the Maximum Principle is not applicable; the topology of the plane differs from the topology of the straight line. Nevertheless, it is possible to define geometrically domains in the function space that are invariant under (1.1). Now an important role is played not by constants, but by special time-independent periodic solutions of (1.1) which correspond to minimal cycles of a corresponding Jacobian metric; the cycles determine "holes" in the plane. These holes create a "soft obstacle" for dynamics (see Remark 
3.6); but if energy is bounded, this obstacle can't be jumped over and creates a topological restriction on dynamics and thus defines invariant domains in the function space. Here we continue research started in [1], [4], [5].

In [1] it was proved that if the graph of $F(u)$ has large enough bumps, one can introduce a non-trivial topological space $D^{\prime}$ in the $u$-plane. The dynamical system $\mathcal{S}_{t}$ generated by (1.1) that takes $u(x, 0)$ to $u(x, t)$, which is defined on $x$-periodic functions with period $L$ with bounded energy has topologically conserved quantities $b=h(u(t))$ where $b$ is an element of the fundamental group of the introduced space. The maximal complexity of $b$ depended on the size of the bumps and the bumps were assumed to be large if the energy (and complexity) of solutions is large. In [4], [5] a connection was shown between the homotopy conservation laws and properties of the Jacobian metric $\sqrt{2 F} d s$. In the present paper we study the case when the energy

$$
\mathcal{E}(u)=\int_{0}^{L} \frac{1}{2}\left|\partial_{x} u\right|^{2}+F(u) d x
$$

of solutions and their complexity is arbitrarily large or infinite (thanks to unboundedness of $L$ ) and describe topological obstacles in intrinsic terms of the Jacobian metric.

Steady-state solutions of (1.1) play important role in the dynamics. We consider bounded solutions of the steady-state equation

$$
\partial_{x}^{2} U-F^{\prime}(U)=0
$$

which satisfy the Hamiltonian conservation law

$$
\left|\partial_{x} U(x)\right|^{2} / 2-F(U(x))=0 \forall x
$$

that is $U(x), \partial_{x} U(x)$ lie on a fixed level surface in $\mathbf{R}^{4}$ of the Hamiltonian. We take $F(u)=F_{0}(u)+\mu, F_{0}(u) \geq 0$, so (1.3) fixes the arbitrary constant $\mu$ in the definition of the potential $F$.

The main object which is responsible for existence of extremely many stable spatial patterns is a minimal cycle. We consider in this paper the case when there exist several minimal cycles, that is closed geodesics $C_{i}=\partial D_{i}$ which encircle non-intersecting domains $D_{i}$. The cycles are contained inside a larger domain $D_{0}$ encircled by an external minimal geodesic $C_{0}$. We give a very simple explicit sufficient condition for existence of a minimal cycle $C_{i}$. Let $C^{3}, C^{2}, C^{1}$ be three circles encircling disks $D^{1} \subset D^{2} \subset D^{3}$ centered at a point $P$ with radii $r_{1}=r /(\pi+1), r_{2}=r, r_{3}=R$ respectively. Let $F(u) \geq M$ in $D^{2} \backslash D^{1}$ and $F(u) \leq m$ on $C^{3}$. If $\sqrt{M} r /(\pi+1)>R \pi \sqrt{m}$ then there 
exists a minimal cycle $C_{i}=\partial D_{i}$ encircling $D_{i}, C^{1} \subset D_{i}$. Geometrically this means that if this condition is fulfilled, the surface corresponding to the Jacobian metric forms a mushroom which grows on the plane, and the minimal cycle encircles its leg. This condition gives in particular a simple criterion for existence of a periodic solution of (1.2). An external minimal cycle $C_{0}$ which encircles $D_{0}$ exists if $F(u)$ has also a "bump" at infinity, for example if $F(u)|u|^{2} \rightarrow \infty$ as $|u| \rightarrow \infty$.

We consider the domain $D^{\prime \prime} \subset \mathbf{R}^{2}, D^{\prime \prime}=D_{0} \backslash \cup_{i=1}^{n} \bar{D}_{i}$. The fundamental homotopy group $\pi_{1}\left(D^{\prime \prime}, \sigma\right)=\pi_{1}$ of the set $D^{\prime \prime}$ is a free group with $n$ generators $g_{i}$ corresponding to counterclockwise cycles in $D^{\prime \prime}$ around points $P_{i} \in D_{i}$ starting from and ending in a fixed closed contractible base set $\sigma$ (this set may consist of one base point $P_{*}$ ); cyclic permutations of words in the group correspond to homotopy classes of closed curves without a designated set $\sigma$; the set of classes is denoted by $\pi_{1}^{\prime}$. A generalized homotopy element $b \in \pi_{1}^{\infty}$ is given as a formal infinite product (irreducible word) $b=\prod_{l=-\infty}^{\infty} g_{i_{l}}^{k_{l}}$ without cancellations. Elements of $\pi_{1}$ are represented by finite irreducible words.

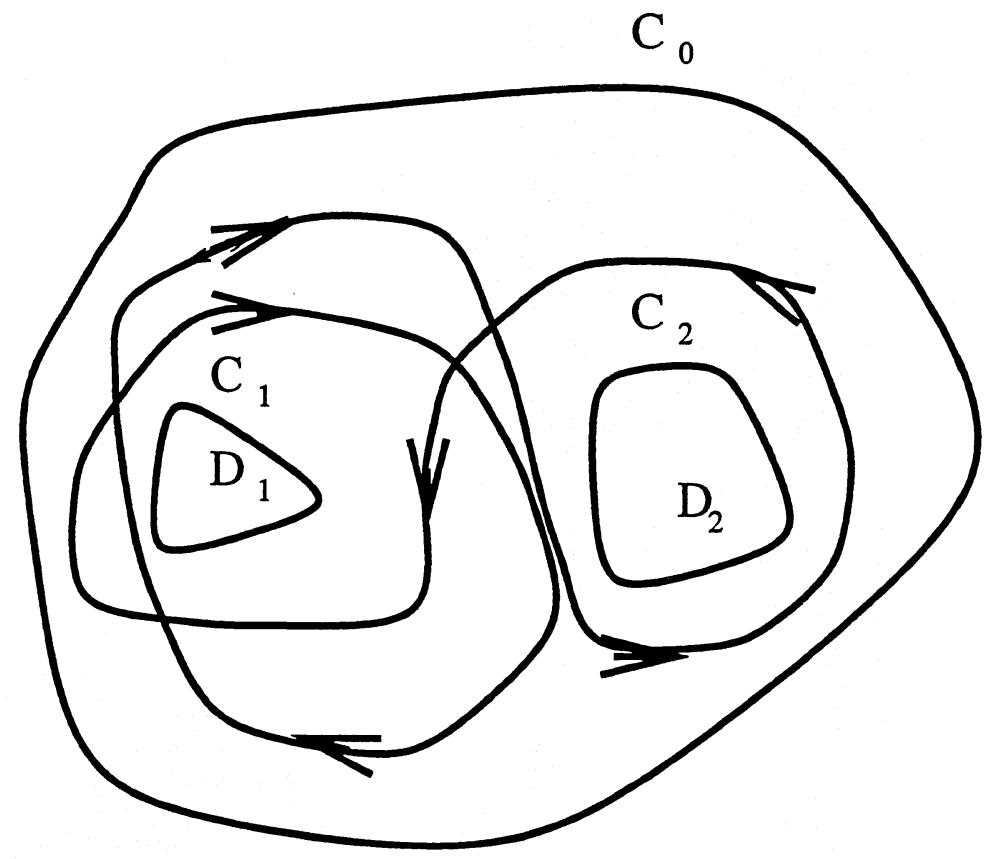

Figure 1: A curve of class $g_{1}^{-2} g_{2}^{1}$ 
We construct solutions with a prescribed homotopy type $b \in \pi_{1}^{\infty}$ using minimizers of Jacobian length. When the interval is infinite we prove for any given $b$ existence of stable steady-state solutions $U(x),-\infty<x<\infty$ of the equation (1.2) which belong to generalized homotopy classes $b \in \pi_{1}^{\infty}$, and finite restrictions of which minimize Jacobian length of the restriction. Note that in geometry existence of minimal geodesics on a manifold in every class of the fundamental group of the manifold is well-known starting from Hadamard (see [8], [9], [14], [16], [21], [22] and references therein). The strong influence of "big bumps" of the Riemannian metric on the manifold on the topology of geodesics is also well known (see [8],[9]). Geodesics which correspond to infinite products of generators are related to minimal geodesics in a cover of the manifold (see [8], [9] and references therein). The most deeply studied cases are the case of geodesics on a torus $\mathbf{T}^{2}$ and on surfaces of constant negative curvature (see [3], [15]); in the mentioned cases topology of the manifold is non-trivial from the very beginning. A variational approach was recently applied in [10], [11] to construct chaotic solutions of a Hamiltonian system on a manifold using the topology of the manifold.

The natural Jacobian metric corresponding to the potential $F(u)$ from (1.1) is defined on the plane $\mathbf{R}^{2}$, apriori the topology in the $u$-plane is trivial; also we do not assume any kind of hyperbolicity. Our paper is not aimed at studying properties of geodesics, but we are trying to use ideas and notions from geometry to study dynamics of curves in the plane generated by (1.1). Since the dynamics of (1.1) cannot be reduced to geodesics (geodesics correspond to equilibria of the parabolic flow), the main point of our research is to find robust properties of geodesics and robust relations between properties of geodesics and properties of general curves which are close to them; these relations have to be stable under the dynamics of (1.1). We managed to do that through introduction of a non-trivial topology in the $u$-plane related to existence of minimal cycles.

Together with steady-state solutions $U(x)$ - minimizers with a given homotopy type $b$ - we consider initial data $u(0)$ which are close to $U$ in the sup-norm of $\mathcal{C}(\mathbf{R})$ and which have local energy density close to that of $U$, namely

$$
\left.\int_{-\infty}^{\infty}|F(u(0, x))+| \partial_{x} u(0, x)\right|^{2} / 2-F(U(x))-\left|\partial_{x} U(x)\right|^{2} / 2 \mid d x \leq \beta .
$$

We prove that the generalized homotopy type $b$ is preserved under dynamics $u(t)$ of (1.1) (in fact $\beta$ may be not small if $F(u)$ is large enough inside $D_{i}$ ). We show that an approximate Hamiltonian conservation law holds for 
solutions of (1.1):

$$
\int_{-\infty}^{\infty}\left(\sqrt{2 F_{0}(u(x, t))+2 \mu}-\left|\partial_{x} u(x, t)\right|\right)^{2} d x \leq 2 \theta \quad \forall t \geq 0 .
$$

Here $\theta$ does not depend on $t$; convergence of (1.4) implies that

$$
2 F_{0}(u(x, t))+2 \mu-\left|\partial_{x} u(x, t)\right|^{2}
$$

can be not small only on a subset of the real line with a finite Lebesgue measure; note that (1.3) does not hold strictly for non-equilibrium solutions of (1.1).

So we classify essentially different invariant classes of (1.1) by two parameters: Hamiltonian value $\mu=\left|\partial_{x} U\right|^{2} / 2-F_{0}(U)$, which is a continuous parameter (clearly convergence of (1.4) fixes $\mu$ for solutions of (1.1) like (1.3) fixes it for solutions of (1.2); and by the generalized homotopy type $b \in \pi_{1}^{\infty}$ which is a parameter of spatial chaos. We study the relation between the homotopy complexity of the solutions restricted to an interval $-L<x<L$ and the length of this interval. The number $N(L)$ of homotopy different restrictions of solutions to a segment of length $2 L$ can be estimated in terms of quantities similar to entropy. We introduce the following characteristics of complexity:

$$
0<h_{*}=\lim \inf _{L \rightarrow \infty} \ln N(L) /(2 L) \leq \lim \sup _{L \rightarrow \infty} \ln N(L) /(2 L)=h^{*} .
$$

We call $h^{*}$ and $h_{*}$ upper and lower complexity respectively.

As an example we consider the case of a special potential when the potential $F(u)=F(u, d)$ is constant outside two very narrow circular rings with width $d$ and has a small variation in the ring. In this case $\left|h^{*}-h_{0} \sqrt{2 \mu}\right|+$ $\left|h_{*}-h_{0} \sqrt{2 \mu}\right|=O(d)$ and $h_{0}$ is a solution of the equation

$$
e^{-h_{0} w}+e^{-h_{0} y}+e^{-h_{0} z}=1
$$

where $w, y, z$ are lengths of common tangents to the two circles (in the limit $d=0$ ) and circular arcs between them (see Section 7 for details). To find this estimate we used topological entropy of the suspended flow (see [25]). Since spatially periodic solutions are of special interest, we estimate (in the case $d=0)$ their number $N_{p e r}(L)$. The number $N_{p e r}(L)$ of homotopy different spatially periodic solutions with period not greater than $L$ is of order

$$
N_{p e r}(L) \sim \frac{e^{h_{0} L \sqrt{2 \mu}}}{h_{0} L \sqrt{2 \mu}}
$$


for large $L$. Note that we consider the case when the diffusion coefficient multiplying $\partial_{x}^{2} u$ is $\nu=1$; the general case is reduced to this by changing $x$ for $\sqrt{\nu} x$. So in the above formula $L$ is a dimensionless length related to the length scale $\sqrt{\nu}$. In case $\nu \neq 1$ one has to replace $\mu$ by $\mu / \nu$.

\section{Reaction-diffusion systems with a positive potential.}

We consider the system (1.1) where $F(u)$ is a potential which has the properties described in the introduction, in particular $F(u)=F_{0}(u)+\mu, F_{0} \geq$ $0, \mu>0$ with a fixed $\mu$. So

$$
F(u) \geq \mu>0, \quad \forall u \in \mathbf{R}^{2} .
$$

Later we show that it suffices to impose this condition only for a bounded set of $u$ rather than for all $u$.

We impose either $L$-periodic boundary conditions

$$
u(0, t)=u(L, t)
$$

or Dirichlet boundary conditions

$$
u(0, t)=p_{1}, u(L, t)=p_{2}
$$

Later we consider the equation on the infinite interval.

The energy of a solution on a finite interval is given by the formula

$$
\mathcal{E}(u)=\int_{0}^{L}\left[\frac{1}{2}\left|\partial_{x} u\right|^{2}+F(u)\right] d x
$$

The energy equation for solutions of (1.1) with periodic or Dirichlet boundary conditions is

$$
\mathcal{E}(u(T))-\mathcal{E}(u(0))=-\int_{0}^{T} \int_{0}^{L}\left|\partial_{t} u\right|^{2} d x d t
$$

The norm in Sobolev spaces $H_{s}$ is defined by $\|u\|_{s}^{2}=\left\|\left(-\partial_{x}^{2}\right)^{s / 2} u\right\|^{2}+\|u\|^{2}$ where $\|u\|$ is the $\mathcal{L}_{2}$-norm of $u$. The space $\mathcal{C}([0, L])$ has the usual sup-norm. By the Sobolev embedding theorem $H_{1}([0, L]) \subset \mathcal{C}([0, L])$.

The following two theorems hold for equation (1.1) with periodic or Dirichlet boundary conditions. 
Theorem 2.1. For any initial data $u(0) \in H_{1}([0, L])$ which satisfies either periodic (2.2) or Dirichlet (2.3) boundary conditions there exists a unique solution $u(t)=u(x, t), u(t) \in H_{2}, t>0$, of (1.1) with periodic (2.2) or Dirichlet (2.3) boundary conditions respectively. It satisfies the energy estimate

$$
\mathcal{E}(u(t)) \leq \mathcal{E}(u(0)) \quad \forall t \geq 0 .
$$

The proof of existence is based on the Galerkin method and is standard, see [6]; the inequality follows from (2.4).

Therefore (1.1) generates a semigroup of operators $\mathcal{S}_{t}: u(0) \rightarrow u(t)$ which acts in $H_{1}([0, L])$.

The following theorem is standard, see [6], [13].

Theorem 2.2. For every $\beta>0$ and every non-empty closed invariant subset $E(\beta) \subset\{\mathcal{E}(u) \leq \beta\}$ bounded in $\mathcal{C}([0, L])$ the semigroup $\mathcal{S}_{t}$ generated in $H_{1}$ by the equation (1.1) has a global attractor $\mathcal{A}(\beta)$ which contains an equilibrium point $U$ such that the energy $\mathcal{E}(U)=\inf _{u \in E(\beta)} \mathcal{E}(u)$. In particular, for every bounded in $\mathcal{C}([0, L])$ solution $u(t), t>0$ of $(1.1)$ there exists a solution $U$ of (1.2) which belongs to the omega-limit set of $u(t)$.

\section{Minimal cycles.}

We introduce the Jacobian (or Fermat-Maupertuis) functional

$$
\mathcal{J}(u)=\int_{0}^{L} \sqrt{2 F(u)}\left|\partial_{x} u\right| d x
$$

the value of this functional is determined by the graph of the curve $u$ and does not depend on its $x$-parameterization, so

$$
\mathcal{J}(u)=\int_{s_{1}}^{s_{2}} \sqrt{2 F(u(s))}|d u(s)| .
$$

Here $u(s)=u(x(s)), \int_{s_{1}}^{s_{2}}|d u(s)|$ is the Euclidean length of the curve. If the natural parametrization $|d u(s)|=d s$ is used, the Euclidean length equals $s_{2}-s_{1}, s_{1}$ can be taken arbitrarily. Here $x(s)$ is a monotone function, $x\left(s_{1}\right)=0, x\left(s_{2}\right)=L$. We consider functions $u(x(s))$ with bounded variation.

Very often we use a parametrization which does not coincide with the natural and take $s_{1}=0, s_{2}=1$. For any curve, for which such an integral is not defined, we put $\mathcal{J}(u)=+\infty$. 
The functional $\mathcal{J}$ determines a Jacobian length and a Riemannian metric on $\mathbf{R}^{2}$ with the tensor $\sqrt{2 F} \delta_{i j}$ where $\delta_{i j}$ is the Kronecker symbol. Jacobian distance between two points $Q_{1}, Q_{2} \in \mathbf{R}^{2}$ is given by $\operatorname{dist} \mathcal{J}\left(Q_{1}, Q_{2}\right)=\inf _{u} \mathcal{J}(u)$ where $u(s)$ connects $Q_{1}, Q_{2}$.

Very often, abusing notations, we write $u(s)$ instead of $u(x(s))$; the parametrization by $s$ is not necessarily the natural Euclidean parametrization. We also use the letter $u$ to denote functions $u(x)$ as well as points in the $u$-plane. The sense of notation is clear from context. Obviously,

$$
\mathcal{J}(u)+\frac{1}{2} \int_{0}^{L}\left(\sqrt{2 F(u(x))}-\left|\partial_{x} u\right|\right)^{2} d x=\mathcal{E}(u)
$$

and

$$
\mathcal{J}(u) \leq \mathcal{E}(u) .
$$

Definition 3.1. Let a smooth simple Jordan curve $C_{i}$ bound a domain $D_{i}$, $C_{i}=\partial D_{i}$ (we always take open domains $D_{i}$ and denote by $\bar{D}_{i}$ their closure). It is called a minimal cycle if it has the following minimality property. For any closed cycle $\Gamma$ which lies in an $\epsilon$-neighborhood $O_{\epsilon}\left(C_{i}\right)$ of the cycle in the $u$-plane, $\Gamma \subset O_{\epsilon}\left(C_{i}\right) \cap \bar{D}_{i}$, once encircles $P_{i} \in D_{i} \backslash \bar{O}_{\epsilon}\left(C_{i}\right)$ and has a point $Q$ strictly inside $D_{i}$ at distance $\epsilon_{1}$ from $C_{i}$ the following inequality holds: $\mathcal{J}(\Gamma) \geq \mathcal{J}\left(C_{i}\right)+\epsilon_{2}$ where $\epsilon_{2}>0$ depends on $\epsilon_{1}$ and does not depend on $\Gamma$.

Definition 3.2. A cycle $C_{i}$ encircling $D_{i}$ is called $\theta$-stable (with $\theta>0$ ) with respect to a cycle $C_{i}^{1}=\partial D_{i}^{1}, \bar{D}_{i}^{1} \subset D_{i}$, if for any curve $\Gamma^{0}=u(x), x_{1} \leq$ $x \leq x_{2}$ which lies in $\bar{D}_{i} \backslash D_{i}^{1}$ and $\Gamma^{0} \cap C_{i}^{1} \neq \emptyset$ there exists a homotopy $\Gamma^{t}, 0 \leq t \leq 1$ of this curve inside the ring $\bar{D}_{i} \backslash D_{i}^{1}$ which does not change points of $\Gamma^{0} \cap C_{i}$ such that $\Gamma^{1} \subset C_{i}, \mathcal{J}\left(\Gamma^{0}\right) \geq \mathcal{J}\left(\Gamma^{1}\right)+\theta$.

Remark 3.1. A minimal cycle $C_{i}$ is $\epsilon_{2}$-stable with respect to a cycle $C_{i}^{1}$ which can be chosen arbitrary close to $C_{i}$, with $\epsilon_{2}$ depending on the distance between $C_{i}^{1}$ and $C_{i}$. This follows from the following lemma.

Lemma 3.1. Let $C_{i}$ be a minimal cycle which encircles $D_{i}$. Let $u^{0}(x), x_{1}<$ $x<x_{2}$ be a curve which lies in $O_{\epsilon}\left(C_{i}\right) \cap D_{i}$, with endpoints on the cycle $u^{0}\left(x_{1}\right)=Q_{1} \in C_{i}, u^{0}\left(x_{2}\right)=Q_{2} \in C_{i}$ and one point $u^{0}\left(x_{0}\right)=Q$ strictly inside $C_{i}$ at distance $\epsilon_{1}>0$ from $C_{i}$. Than there exists a homotopy $u^{t}(x)$ in $O_{\epsilon}\left(C_{i}\right) \cap \bar{D}_{i}$ which transforms the curve into the curve $u^{1}(x)$ with the same endpoints $u^{1}\left(x_{1}\right)=Q_{1}, u^{1}\left(x_{2}\right)=Q_{2}$ which takes values in $C_{i}$. There exists a number $\epsilon_{2}>0$ which depends only on $\epsilon_{1}$ such that $\mathcal{J}\left(u^{1}\right) \leq \mathcal{J}\left(u^{0}\right)-\epsilon_{2}$. The same is true for closed $u^{0}$ without points on $C_{i}$. 
Proof. The reason we need a proof is that in the definition of a minimal cycle the curve $\Gamma$ is a cycle which once turns around $D$, and here we may have many turns.

Since the ring $A=\bar{O}_{\epsilon}\left(C_{i}\right) \cap \bar{D}_{i}$ is homeomorphic to the product $C_{i} \times[0,1 / 2]$ we take the homotopy $\mathcal{H}(t), 0 \leq t \leq 1 / 2$ defined on the ring $A$ which shrinks $[0,1 / 2]$ to $\{0\}$; it is the identity function on the boundary $C_{i}$, $\mathcal{H}(0)(A)=A, \mathcal{H}(1 / 2)(A)=C_{i}=C_{i} \times\{0\}$. Let $\Gamma^{\prime}$ be the arc of $C_{i}$ with endpoints $Q_{1}, Q_{2}$ which forms together with $u^{0}$ an oriented cycle $G^{0}$ (cycles $C_{i}$ are always oriented counterclockwise, orientation of $u^{0}$ is determined by its parameterization). The curves $G^{0}$ and $G^{1}=\mathcal{H}(1 / 2)\left(G_{0}\right)$ have the same homotopy type $g_{i}^{\alpha}$ where $\alpha$ equals to the rotation number around $D_{i}$. The closed curve $G^{0}$ is parameterized by $x_{1} \leq x \leq x_{3}\left(x_{2} \leq x \leq x_{3}\right.$ is a parameterization of $\left.\Gamma^{\prime}\right)$. We identify $x_{1}$ and $x_{3}$. The curve $G^{1}(s)=\mathcal{H}(1 / 2)\left(G^{0}(s)\right)$ has the induced parameterization. We introduce the coordinate $\phi$ on $C_{i}$ which is proportional to the Euclidean distance from $u^{0}\left(x_{1}\right)$ along the curve in the counterclockwise direction and is normalized so that $\phi$ and $\phi+2 \pi$ are coordinates of the same point. The standard curve $G^{2} \subset C_{i}$ is obtained by passing $\alpha$ times along $C_{i}$ with a non-zero constant speed, it is given by the formula $\phi=\phi_{2}(x)=2 \pi \alpha\left(x-x_{1}\right) /\left(x_{3}-x_{1}\right)$; we use the same parametrization for $\Gamma^{\prime}$. We have $\mathcal{J}\left(G^{2}\right)=|\alpha| \mathcal{J}\left(C_{i}\right)$. There is a homotopy in $C_{i}$ which connects $G^{1}$ and $G^{2}$ (since they are from the same homotopy class; the fundamental groups $\pi_{1}\left(\mathbf{R}^{2} \backslash D_{i}\right)$ and $\pi_{1}\left(C_{i}\right)$ are naturally isomorphic, they are given by the rotation number). The curves $G_{1}$ and $G_{2}$ are written using the coordinate $\phi$ as $\phi_{1}(x)$ and $\phi_{2}(x)$ respectively with $\phi_{1}\left(x_{1}\right)=\phi_{2}\left(x_{1}\right)$ by definition, $\phi_{1}\left(x_{3}\right)=\phi_{2}\left(x_{3}\right)=\phi_{1}\left(x_{1}\right)+2 \alpha \pi$ since they have the same rotation number. The homotopy connecting them is given by $t \phi_{1}(x)+(1-t) \phi_{2}(x)$; it is the identity function on $x_{2} \leq x \leq x_{3}$.

We take as $u^{1}$ the curve which represents $G^{2}$ and is composed of the arc $\Gamma^{\prime \prime}=C_{i} \backslash \Gamma^{\prime}$ and $|\alpha|-1$ cycles $C_{i}$. Its length equals

$$
\mathcal{J}\left(u^{1}\right)=|\alpha| \mathcal{J}\left(C_{i}\right)-\mathcal{J}\left(\Gamma^{\prime}\right) .
$$

Now we give a lower estimate of $\mathcal{J}\left(G^{0}\right)$ :

$$
\mathcal{J}\left(G^{0}\right) \geq|\alpha| \mathcal{J}\left(C_{i}\right)+\epsilon_{2}
$$

This estimate follows from the definition of a minimal cycle when $|\alpha|=1$ ( closed curves with $|\alpha|=1$ we call elementary). In the general case we will show that $G^{0}$ includes at least $|\alpha|$ elementary curves, and one of them passes through $Q$; this yields the estimate. First we make general remarks. Consider a closed curve $u(s), s_{0} \leq s \leq s_{6}$ in $D_{i}$ which has a finite Jacobian 
length, lies in the ring $A$ and has a non-trivial homotopy type $b=g_{i}^{\alpha}$ with a fixed $\alpha \neq 0$ with respect to a point $P_{i} \in D_{i} \backslash A$ (we denote the set of such curves by $\Xi_{0}$ ). The curve may have self-intersections; if $|\alpha|>1$ the curve must have self-intersections, since by the Jordan's theorem a simple curve is a cycle for which $\alpha= \pm 1$.

An interval $\left[s_{4}, s_{5}\right] \subset\left[s_{0}, s_{6}\right]$ is called a rest interval if $u(s)=u\left(s_{5}\right) \forall s \in$ $\left[s_{4}, s_{5}\right]$; for every rest interval we can find a maximal rest interval which contains it. An interval $\left[s_{1}, s_{2}\right] \subset\left[s_{0}, s_{6}\right]$ is called a loop interval if $u\left(s_{2}\right)=$ $u\left(s_{1}\right)$ and there exists $s_{0} \in\left[s_{1}, s_{2}\right]$ such that $u\left(s_{0}\right) \neq u\left(s_{1}\right)$. We call the loop interval $\left[s_{1}, s_{2}\right]$ simple if for every two points $s_{5}, s_{4}$ such that $s_{1} \leq s_{4}<s_{5} \leq$ $s_{2}$ the equality $u\left(s_{5}\right)=u\left(s_{4}\right)$ implies that $\left[s_{4}, s_{5}\right]$ is a rest interval. If the homotopy class on a loop interval $\left[s_{1}, s_{2}\right]$ with respect to $P_{i}$ is trivial we call this interval non-essential (see Fig. 2 where a loop which corresponds to a non-essential loop interval is shown).

If we have a loop interval $\left[s_{1}, s_{2}\right]$, we can modify a curve by a cut-off operation eliminating the loop, that is by putting $u(s)=u\left(s_{1}\right), s_{1} \leq s \leq s_{2}$ without changing the curve outside the loop interval.

The cut-off operation does not increase Jacobian length and variation; if the interval is non-essential it preserves the homotopy type. Such operation allows to obtain from a given $u(s)$ a curve $\widehat{u}(s)$ which has the same type and has no non-essential loop intervals. It is clear that if the original curve has a finite number of self-intersections, we can replace non-essential loop intervals by rest intervals in a finite number of steps.

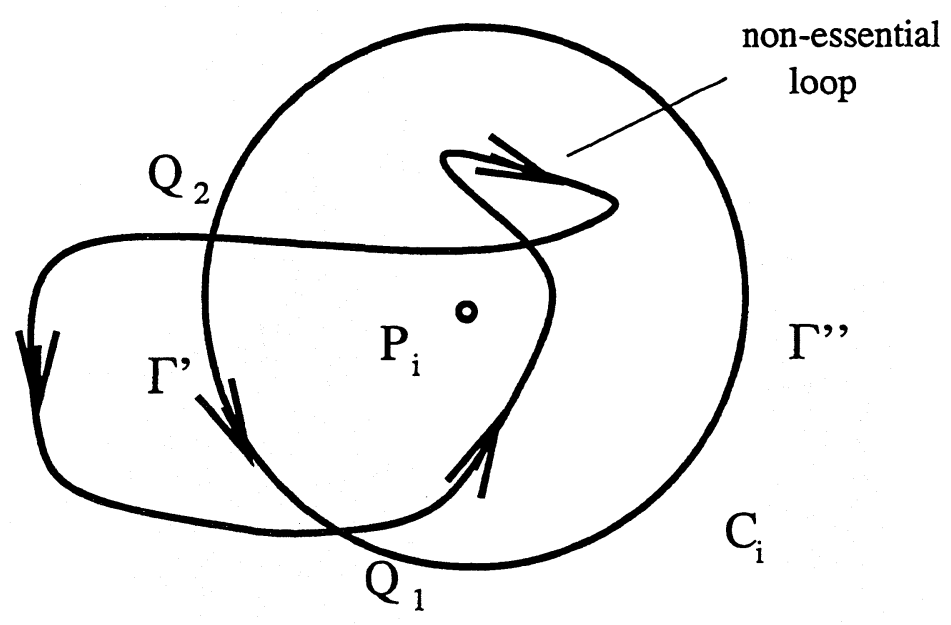

Figure 2. 
In the general case we introduce a partial ordering in the set $\Xi_{0}$. We say that $u>>v$ if $u(s), v(s)$ are defined on the same interval $\left[s_{0}, s_{6}\right]$, every rest interval or non-essential loop interval of $u$ is a rest interval of $v$ and $u(s)=v(s)$ outside rest intervals of $v$. Starting with a given $u$ and applying the above modification we obtain in the limit as a minimal element $\widehat{u}(s)$ which has no non-essential loop intervals and the corresponding curve $\widehat{G}^{0}$. The homotopy type of $G^{0}$ is not changed, $h\left(\widehat{G}^{0}\right)=h\left(G^{0}\right)=\alpha$.

Every simple loop interval of $\widehat{u}(s)$ has a graph which is a simple Jordan curve without self-intersections and by Jordan's theorem the loop belongs to the class $g_{i}^{ \pm 1}$. Since the type of an essential loop is $g_{i}^{ \pm 1}$, after applying the cut-off operation to a simple (essential) loop interval the homotopy type $\alpha$ is changed by \pm 1 . The Jacobian length of a deleted simple loop is not less than $\mathcal{J}\left(C_{i}\right)$ by definition of a minimal cycle. We have self-intersections and may continue the cut-off operations as long as $|\alpha|>1$ by Jordan's theorem. After $N-1$ cut-off operations we have only one loop interval (the number $N$ of such operations is finite since $v=\widehat{u}$ has a finite length), and the resulting curve is of the class $\alpha= \pm 1$. Therefore we obtain the inequality $\mathcal{J}\left(G^{0}\right) \geq N \mathcal{J}\left(C_{i}\right)$. We need at least $|\alpha|$ subtractions of \pm 1 from $\alpha$ to get zero, so $N \geq|\alpha|$. Therefore we get $\mathcal{J}\left(G^{0}\right) \geq|\alpha| \mathcal{J}\left(C_{i}\right)$, which is a weaker version (with $\epsilon_{2}=0$ ) of (3.4).

Consider now the point $s=s_{*}, u\left(s_{*}\right)$ at distance $\epsilon_{1}$ from $C_{i}$, it lies on one of the simple loops. We have $s_{*} \in\left[s_{1}, s_{2}\right]$ with $u\left(s_{1}\right)=u\left(s_{2}\right)$. The loop may be non-essential, therefore we take such $s_{3} \geq s_{2}, u\left(s_{3}\right)=u\left(s_{1}\right)$ that the homotopy type of $u(s)$ on $\left[s_{1}, s_{3}\right]$ is $g_{i}^{ \pm 1}$; the Jacobian length of this loop $G^{0 \prime \prime}$ is not less than $\mathcal{J}\left(C_{i}\right)+\epsilon_{2}$ by definition of a minimal cycle. Cutting off this loop we obtain a curve $G^{0^{\prime}}$ of class $\alpha \mp 1$. Applying the weak version of (3.4) to $G^{0^{\prime}}$ we get $\mathcal{J}\left(G^{0^{\prime}}\right) \geq|\alpha \mp 1| \mathcal{J}\left(C_{i}\right)$. Since $\mathcal{J}\left(G^{0}\right)=\mathcal{J}\left(G^{0^{\prime}}\right)+\mathcal{J}\left(G^{0^{\prime \prime}}\right)$ we obtain (3.4). Comparing (3.4) with (3.3) we obtain the assertion of the lemma.

Consider now the case when we have several minimal cycles. From now on we fix the following notations. We denote by $D_{i}, D_{i}^{1}, i=1, \ldots, n n$ pairs of open domains in the plane, the boundary $\partial D_{i}=C_{i}, \partial D_{i}^{1}=C_{i}^{1}$ of every domain is a simple Jordan curve; $\epsilon$-neighborhoods of the domains do not intersect: $O_{\epsilon}\left(D_{i}\right) \cap O_{\epsilon}\left(D_{j}\right)=\emptyset$ when $1 \leq i<j \leq n$. We denote by $P_{i}$ a point such that $P_{i} \in D_{i}^{1} \subset \bar{D}_{i}^{1} \subset D_{i}$. We assume that $\partial D_{i}^{1}$ lies in an $\epsilon$-neighborhood of $\partial D_{i}$ (see Remark 3.1). By $C_{i}$ we denote cycles which are minimal and $\theta$-stable with resect to $C_{i}^{1}, i=1, \ldots, n$. (Sufficient conditions for existence of such cycles are given later in this section). 
Definition 3.3. We denote by $\Xi$ the set of closed continuous curves $u(s)$ (parametrized by $s \in[0,1], u(0)=u(1)$ ) which do not intersect $D_{i}^{1}$ and have finite Jacobian length. We denote for $b \in \pi_{1}^{\prime}\left(\mathbf{R}^{2} \backslash \cup_{1}^{n} D_{i}^{1}\right)$ by $\Xi_{b}$ the set of curves from $\Xi$ which belong to $b$ and put

$$
\ell^{*}(b)=\inf _{u \in \Xi_{b}} \mathcal{J}(u)
$$

The same definition applies to curves with fixed endpoints when $b \in \pi_{1}$.

Definition 3.4. We denote by $\Xi^{\prime}([0, L])$ and $\Xi_{b}^{\prime}([0, L])$ the set of functions $u \in H_{1}([0, L])$ which have graphs from $\Xi$ and $\Xi_{b}$ respectively.

Theorem 3.1. Let $C_{i}, i=1, \ldots, n$ be the $\theta$-stable cycles described above; let $b \in \pi_{1}^{\prime}$ be the homotopy class of closed curves. Then the sets $\Xi_{b}^{\prime}([0, L]) \cap$ $\{\mathcal{E}(u) \leq \mathcal{L}\}$ where $\mathcal{L}<\ell^{*}(b)+\theta$ are invariant with respect to the flow defined in $H_{1}([0, L])$ by the parabolic equation (1.1) with periodic boundary conditions. Similar statement holds for the Dirichlet boundary conditions when $b \in \pi_{1}$. The following approximate Hamiltonian conservation holds:

$$
\int_{0}^{L}\left(\sqrt{2 F(u(x))}-\left|\partial_{x} u\right|\right)^{2} d x \leq 2 \theta
$$

Proof. We consider the case of periodic boundary conditions. Consider the set $\Xi_{b} \cap\{\mathcal{J}(u) \leq \mathcal{L}\}$ where $\mathcal{L}<\ell^{*}(b)+\theta$. According to Definition 3.2 every curve $u(x)$ from this set lies strictly inside $\mathbf{R}^{2} \backslash D_{i}^{1}, u(x) \cap C_{i}^{1}=\emptyset \forall i, u, x$. Indeed, assume the contrary, namely let there exist a curve $u$ such that $u\left(s_{*}\right) \in C_{i}^{1}$. Since $C_{i}^{1} \subset D_{i}$, we have two possibilities. Either we can find an interval $\left[s_{1}, s_{2}\right]$ containing $s_{*}$ such that $u(s) \in D_{i}$ for $s_{1}<s<s_{2}, u\left(s_{1}\right)=$ $Q_{1} \in C_{i}, u\left(s_{2}\right)=Q_{2} \in C_{i}$, or the curve does not intersect $C_{i}$. We consider the first case. We fix $s_{1}, s_{2}$ and denote the curve $\Gamma_{0}$ on the segment $\left[s_{1}, s_{2}\right]$ by $u_{*}(s)$. By $\theta$-stability we have a homotopy on $\left[s_{1}, s_{2}\right]$ which takes $u_{*}(s)$ into $u_{*}^{1}(s)$ with $\mathcal{J}\left(u_{*}\right) \geq \mathcal{J}\left(u_{*}^{1}\right)+\theta$. We do not change $u(s)$ outside $\left[s_{1}, s_{2}\right]$, and $u_{*}^{t}(s), t \in[0,1]$ do not take values outside $D_{i}$ and therefore do not intersect $C_{j}^{1}, j \neq i$. So we have a homotopy in $\mathbf{R}^{2} \backslash \cup_{1}^{n} D_{i}^{1}$ of $u(s)$ into $u^{1}(s)$ which decreases the Jacobian length by $\theta$, so $\mathcal{J}\left(u^{1}\right)<\ell^{*}(b)$ which contradicts the definition of $\ell^{*}(b)$. In the second case we again use $\theta$-stability and obtain the same contradiction; thus $u(s)$ cannot have points on $C_{i}^{1}$.

Therefore $\Xi_{b}^{\prime}([0, L]) \cap\{\mathcal{E}(u) \leq \mathcal{L}\}$ is invariant with respect to the flow defined in $H_{1}$ by the parabolic equation (1.1). Indeed, $u(t)$ is a continuous function of $t$ and by $(2.5) \mathcal{E}(u(t)) \leq \mathcal{E}(u(0)) \leq \mathcal{L}$. If $u\left(t_{0}\right) \in \Xi_{b}^{\prime}([0, L]) \cap$ 
$\{\mathcal{E}(u) \leq \mathcal{L}\}$ then its graph is at positive distance from all $C_{i}^{1}$; therefore it is true for $t \leq t_{0}+\epsilon$. Hence the set $T$ of $t$ for which $u(t) \in \Xi_{b}^{\prime}([0, L]) \cap$ $\{\mathcal{E}(u) \leq \mathcal{L}\}$ is open. On the other hand, the set $\Xi_{b}^{\prime}([0, L]) \cap\{\mathcal{E}(u) \leq \mathcal{L}\}$ is closed in $H_{1}([0, L])$ since $\mathcal{E}(u)$ is continuous, $H_{1}([0, L]) \subset \mathcal{C}([0, L])$ and the set $\mathbf{R}^{2} \backslash D_{i}^{1}$ is closed. Thanks to continuity of $u(t)$ and closedness of $\Xi_{b}^{\prime}([0, L]) \cap\{\mathcal{E}(u) \leq \mathcal{L}\}$ we conclude that $T$ is closed. Therefore this set is all $t \geq 0$. $\ell^{*}(b)$.

Inequality (3.5) follows from (3.1) since $u(t) \in \Xi_{b}^{\prime}([0, L])$ and $\mathcal{J}(u(t)) \geq$

To make ideas transparent and geometrically obvious, we give now as an example a special type of a potential when minimal cycles are explicitly given. We discuss this example in more detail in Section 6 .

Example 3.1. We fix two points $P_{i}, i=1,2$ in the plane $\left(u_{1}, u_{2}\right)$ at the distance $2 a>0$; fixing the coordinate system we take the points on the $u_{1}$-axis, $P_{1}=(-a, 0), P_{2}=(a, 0)$. We denote by $D_{i}(r)$ discs of radius $r$ centered at the points $P_{i}, D_{i}(r)=\left\{\left|u-P_{i}\right| \leq r\right\}, r<a$. We fix $r_{3}<a$ and assume that the function $F(u)$ in the $r_{3}$-neighborhoods $D_{i}\left(r_{3}\right)$ of $P_{i}$ depends only on $\eta=\left|P_{i}-u\right|$, we denote

$$
F(u)=f(\eta) \quad \text { when } \eta=\left|u-P_{i}\right| \leq r_{3} .
$$

We also assume that there exists $\eta_{0}, 0<\eta_{0} \leq r_{3}$ such that the function $\eta \sqrt{2 f(\eta)}$ has a strict minimum at this point,

$$
\eta \sqrt{2 f(\eta)}>\eta_{0} \sqrt{2 f\left(\eta_{0}\right)}, \forall \eta \in\left[\eta_{0}-\epsilon, \eta_{0}+\epsilon\right], \eta \neq \eta_{0} .
$$

Lemma 3.2. Let (3.7) hold. Then $\left|u-P_{i}\right|=\eta_{0}$ is a minimal cycle.

Proof. Let $(\eta(x), \phi(x))$ be the polar coordinates of a curve in $D_{i}\left(\eta_{0}\right)$. Let $\eta\left(x_{1}\right)=\eta\left(x_{2}\right)=\eta_{0}, \eta(x)<\eta_{0}, x_{1}<x<x_{2}$. One can write the Jacobian length of a piece of the curve in the polar coordinates at $P_{i}$ on the interval when $\eta \leq r_{3}$ as

$$
\mathcal{J}(u)=\int_{x_{1}}^{x_{2}} \sqrt{2 f(\eta)}\left|\partial_{x} u\right| d x=\int_{x_{1}}^{x_{2}} \sqrt{2 f(\eta(x))} \sqrt{\eta^{2}{\phi^{\prime}}^{2}(x)+\eta^{\prime 2}(x)} d x .
$$

First, we can replace the piece of the curve by a curve with monotonely varying $\phi(x)$; indeed, if $\phi(x)$ has a local maximum at $x_{0} \in\left(x_{1}, x_{2}\right)$ we have 
$\phi\left(x_{1}^{\prime}\right)=\phi\left(x_{2}^{\prime}\right)=\phi_{0}$ for some points $x_{1}^{\prime}, x_{2}^{\prime}$ close to $x_{0}$ and we put $\phi(x)=\phi_{0}$ on $\left(x_{1}^{\prime}, x_{2}^{\prime}\right)$. Since

$$
\int_{x_{1}^{\prime}}^{x_{2}^{\prime}} \sqrt{2 f(\eta(x))}\left[\sqrt{\eta^{2}{\phi^{\prime}}^{2}(x)+{\eta^{\prime}}^{2}(x)}-\left|\eta^{\prime}(x)\right|\right] d x>0
$$

the resulting length is smaller. So we assume that $\phi(x)$ is monotone on $\left(x_{1}, x_{2}\right)$. Since it is a cycle, the variation $\Delta_{\phi}$ of $\phi$ is not greater than $2 \pi$.

If we replace $\eta(x)$ by $\eta_{0}$, we replace $\eta^{\prime}$ by 0 and $\sqrt{2 f(\eta) \eta^{2}}$ by $\sqrt{2 f\left(\eta_{0}\right) \eta_{0}^{2}}$, we obtain for the new curve $\widetilde{u}$

$$
\mathcal{J}(u)-\mathcal{J}(\widetilde{u})=\int_{x_{1}}^{x_{2}}\left[\sqrt{2 f(\eta)} \sqrt{\eta^{2}{\phi^{\prime}}^{2}+{\eta^{\prime}}^{2}}-\sqrt{2 f\left(\eta_{0}\right)} \sqrt{\eta_{0}^{2}{\phi^{\prime}}^{2}}\right] d x
$$

which is not less than

$$
\begin{aligned}
\sqrt{2 f\left(\eta_{0}\right) \eta_{0}^{2}} \int_{x_{1}}^{x_{2}}\left[\sqrt{1+\eta_{\phi}^{\prime 2} / \eta^{2}}-1\right]\left|\phi^{\prime}\right| d x \\
+\int_{x_{1}}^{x_{2}}\left[\sqrt{2 f(\eta) \eta^{2}}-\sqrt{2 f\left(\eta_{0}\right) \eta_{0}^{2}}\right]\left|\phi^{\prime}\right| d x .
\end{aligned}
$$

Here $\eta_{\phi}^{\prime}=\eta^{\prime} / \phi^{\prime}=d \eta / d \phi$. The first integral is not less than the difference of two lengths. The second length $\Delta_{\phi} \leq 2 \pi$ equals the variation of the angle $\phi$ and can be considered as the length of the interval $0<\phi<\Delta_{\phi}$ in the $(\phi, \ln \eta)$-plane. The first length is the length of the continuous curve $\ln \eta(\phi)-\ln \eta_{0}$ in this plane. Let $x_{0}$ be a point of maximum of $\mid \ln \eta(\phi(x))-$ $\ln \eta_{0}|,| \ln \left(\eta\left(\phi\left(x_{0}\right)\right)-\ln \eta_{0} \mid=\epsilon_{3}>0\right.$. If the curve intersects the cycle $\eta=\eta_{0}$ this difference is minimal when the curve forms an isosceles triangle and is not less than $2\left[\epsilon_{3}^{2}+\pi^{2}\right]^{1 / 2}-2 \pi$. Therefore,

$$
\mathcal{J}(u)-\mathcal{J}(\widetilde{u}) \geq \epsilon_{2}=2 \sqrt{2 f\left(\eta_{0}\right) \eta_{0}^{2}}\left(\left[\epsilon_{3}^{2}+\pi^{2}\right]^{1 / 2}-\pi\right) .
$$

If the curve does not intersect the cycle $\eta=\eta_{0}$ and lies strictly inside, let $\eta_{1}$ be the maximal value of $\eta$ on the curve. In this case the variation of $\phi$ is $2 \pi$ and the first integral is estimated by $2 \sqrt{2 f\left(\eta_{0}\right) \eta_{0}^{2}}\left(\left[\epsilon_{3}^{2}+\pi^{2}\right]^{1 / 2}-\pi\right)$ with $\epsilon_{3}=\mid \ln \left(\eta\left(\phi\left(x_{0}\right)\right)-\ln \eta_{1} \mid \geq 0\right.$. The second integral in (3.9) is not less than

$$
2\left[\sqrt{2 f_{1}\left(\eta_{1}\right)}-\sqrt{2 f\left(\eta_{0}\right) \eta_{0}^{2}}\right] \mid \pi, f_{1}\left(\eta_{1}\right)=\min _{\eta_{0}-\epsilon \leq \eta \leq \eta_{1}} f(\eta) \eta^{2}
$$


Since $f_{1}\left(\eta_{1}\right)$ is a continuous function and $f_{1}\left(\eta_{1}\right)>f\left(\eta_{0}\right) \eta_{0}^{2}$ for $\eta_{1}<\eta_{0}$ we have

$$
\mid \ln \left(\eta\left(\phi\left(x_{0}\right)\right)-\ln \eta_{1}\left|+2\left[\sqrt{2 f_{1}\left(\eta_{1}\right)}-\sqrt{2 f\left(\eta_{0}\right) \eta_{0}^{2}}\right]\right| \pi \geq \epsilon_{2}\right.
$$

for $\eta\left(\phi\left(x_{0}\right)\right) \leq \eta_{1} \leq \eta_{0}$ with $\epsilon_{2}>0$. This gives the minimality property.

Now we give a sufficient condition for existence of a minimal cycle in a general case.

Definition 3.5. Let $C^{1}=\partial D^{1}, C^{2}=\partial D^{2}$ be two non-intersecting simple Jordan curves (cycles) which encircle domains $D^{1}, D^{2}$ respectively, $D^{1} \subset D^{2}$, let $B=\bar{D}^{2} \backslash D^{1}$ be a closed band with the boundary $\partial B=C^{1} \cup C^{2}$. We say that the size of $B$ is not less than $\beta_{0}$ if the following two conditions are satisfied. First,

$$
\inf _{Q^{1} \in C^{1}, Q^{2} \in C^{2}} \operatorname{dist} \mathcal{J}\left(Q^{1}, Q^{2}\right) \geq \beta_{0}
$$

Second, there exist two sets $B_{1}, B_{2} \subset B$ such that $B \backslash B_{1}$ and $B \backslash B_{2}$ are contractible (a set $X$ is contractible if there is a homotopy of $X$ into $X$ which starts as identity and ends as a constant) and

$$
\inf _{u^{1} \in B_{1}, u^{2} \in B_{2}} \operatorname{dist} \mathcal{J}\left(u^{1}, u^{2}\right) \geq \beta_{0} .
$$

The distances dist $\mathcal{J}$ in both formulas are taken in the band $B$, that is using the curves confined to $B$.

Example 3.2. Let $P_{i}$ be a given point, $F(u) \geq M$ when $\left|u-P_{i}\right| \leq r_{2}$. We take $C_{i}^{1}=\left\{\left|u-P_{i}\right|=r_{1}\right\}, C_{i}^{2}=\left\{\left|u-P_{i}\right|=r_{2}\right\}, r_{1}<r_{2}$. Condition (3.10) takes the form $\sqrt{2 M}\left(r_{2}-r_{1}\right) \geq \beta_{0}$. We introduce polar coordinates $\eta, \phi$ with the origin at $P_{i}$. We take as $B_{1}$ the straight line segment originating at $P_{i}$ at the angle $\phi=0, r_{1} \leq \eta \leq r_{2}$. As $B_{2}$ we take the symmetric segment $\phi=\pi, r_{1} \leq \eta \leq r_{2}$. Obviously, (3.11) takes the form $\pi \sqrt{2 M} r_{1} \geq \beta_{0}$. To maximize $\beta_{0}$ we take $r_{1}=r_{2} /(\pi+1)$. Therefore the size of $B$ for such a metric $\mathcal{J}$ is not less than $\sqrt{2 M} r_{2} \pi /(\pi+1)$.

We will use below the following simple lemma on the Hamiltonian parametrization. 
Lemma 3.3. For a given smooth curve $u(s)$ on which $F(u(s)) \geq \mu$ there exists such a parameterization $u(x), 0 \leq x \leq L$ that

$$
\mathcal{J}(u)=\mathcal{E}(u)
$$

and

$$
\frac{l_{E}(u)^{2}}{2 \mathcal{E}(u)} \leq L \leq \frac{1}{2 \mu} \mathcal{J}(u)
$$

where $l_{E}(u)$ is the Euclidean length of the curve $u$.

Proof. We assume (1.3); by $(3.1) \mathcal{J}(u)=\mathcal{E}(u)$ in this case. From (1.3) we obtain the equation for the Hamiltonian parameterization

$$
\frac{d x}{d s}=\frac{1}{\sqrt{2 F(u(s))}}
$$

where $s$ is the natural Euclidean parametrization of the curve. This gives

$$
L=\int_{s_{1}}^{s_{2}} \frac{d s}{\sqrt{2 F(u(s))}}
$$

Since $F \geq \mu$, we have

$$
L=\int_{s_{1}}^{s_{2}} \frac{\sqrt{2 F(u(s))} d s}{2 F(u(s))} \leq \frac{\mathcal{J}(u)}{2 \mu} .
$$

Hence, we have the second inequality in (3.12). On the other hand,

$$
l_{E}(u)=\int_{0}^{L}\left|\partial_{x} u\right| d x \leq L^{1 / 2}(2 \mathcal{E}(U))^{1 / 2}
$$

and the lemma is proven.

First we consider the case when only one $D_{i}^{1}$ is given, that is $n=1$.

Theorem 3.2. Let $D_{i}^{1}$ be encircled by a band $B$ with size not less than $\beta_{0}$. Let a cycle $C^{3}=\partial D^{3}$ with $D_{i}^{1} \subset D^{3}, \mathcal{J}\left(C^{3}\right)<2 \beta_{0}$. Then there exists a minimal cycle $C_{i}$ encircling $P_{i} \in D_{i}^{1}$, this cycle is $\theta$-stable with respect to $C_{i}^{1}$ with $\theta=2 \beta_{0}-\mathcal{J}\left(C^{3}\right)$. It determines, with the parameterization (1.3), a solution of (1.2). 
Proof. The cycle $C^{3}$ is $\theta$-stable with respect to $C_{i}^{1}$ on curves of class $g_{i}^{ \pm 1}$. Namely, let a cycle $\Gamma_{0}$ which lies in $D^{3} \backslash D_{i}^{1}$ and once encircles $D_{i}^{1}$ have a point $Q_{1} \in C_{i}^{1} \cap \Gamma_{0}$. There are two possibilities. First, there is a point $Q^{\prime} \in \Gamma_{0}$ which is outside $D^{2}$. In this case there is a point $Q_{2} \in C^{2} \cap \Gamma_{0}$. We have two arcs on $\Gamma_{0}$ which connect $Q_{1}$ and $Q_{2}$, their subarcs connect $C_{i}^{1}$ and $C^{2}$ and (3.10) implies $\mathcal{J}\left(\Gamma_{0}\right) \geq 2 \beta_{0}$. The second possibility is $\Gamma_{0} \subset B=\bar{D}^{2} \backslash D_{i}^{1}$. In this case $\Gamma_{0}$ has to intersect $B_{1}$ and $B_{2}$. Indeed, the set $B \backslash B^{1}$ is contractible in $B$ and $\Gamma_{0}$ is not, so we have a point $Q_{1}^{\prime} \in \Gamma_{0} \cap B_{1}$. Similarly, $Q_{2}^{\prime} \in \Gamma_{0} \cap B_{2}$. We have two arcs on $\Gamma_{0}$ which connect $Q_{1}^{\prime}$ and $Q_{2}^{\prime}$ inside $B$ and (3.11) again implies $\mathcal{J}\left(\Gamma_{0}\right) \geq 2 \beta_{0}$. Since $\Gamma_{0}$ once encircles $D_{i}^{1}$ it has the same homotopy type as $C^{3}$, so there is a homotopy connecting them in $\mathbf{R}^{2} \backslash D_{i}^{1}$; since $D_{3} \backslash D_{i}^{1}$ is a retract of $\mathbf{R}^{2} \backslash D_{i}^{1}$ there is a homotopy in $D_{3} \backslash D_{i}^{1}$. Since $\mathcal{J}\left(\Gamma_{0}\right) \geq 2 \beta_{0}$ we obtain $\mathcal{J}\left(\Gamma_{0}\right)-\mathcal{J}\left(C^{3}\right) \geq \theta$ with $\theta=2 \beta_{0}-\mathcal{J}\left(C^{3}\right)$, so $C^{3}$ is $\theta$-stable.

Consider the set $\Xi_{1}^{0}$ of all curves which encircle once $D_{i}^{1}$, that is have a homotopy type $g_{i}^{1} \in \pi_{1}\left(\mathbf{R}^{2} \backslash D_{i}^{1}\right)$, let $\ell^{*}=\ell^{*}\left(g_{i}^{1}\right)$ be the infinum of their Jacobian lengths, we have $\ell^{*} \leq \mathcal{J}\left(C_{i}^{3}\right)<2 \beta_{0}$. We take a smooth cycle $C^{j}, \mathcal{J}\left(C^{j}\right) \leq \ell^{*}+\epsilon_{j}, \epsilon_{j} \rightarrow 0$. Using Lemma 3.3 we parametrize $C^{j}$ to obtain a periodic function $u(x)$ with period $L_{j}$ which satisfies (1.3) and with $\mathcal{E}(u)=\mathcal{J}(u) \leq \ell^{*}+\epsilon_{j}$. If $\beta<\ell^{*}+\epsilon_{j}$ let $\widetilde{E}\left(L_{j}, \beta\right)$ be a subset of $\left\{u \in H_{1}\left(\left[0, L_{j}\right]\right), \mathcal{E}(u) \leq \beta\right\}$ consisting of these functions whose graphs once encircle $D_{i}^{1}$ (belong to $\Xi_{1}^{0}$ ). We can apply Theorem 3.1 to $\widetilde{E}$ since we need $\theta$-stability only with respect to curves of type $g_{i}^{1}$.

By Theorem 3.1 the set $\widetilde{E}$ is invariant: $u(t) \in \widetilde{E}, t \geq 0$; moreover, it is bounded in $\mathcal{C}\left(\left[0, L_{j}\right]\right)$ since from boundedness of $\mathcal{E}(u)$ follows a bound for the Euclidean length of the graph of $u$ and the graph encircles $P_{1}$; therefore the statement of Theorem 2.2 holds. So we have a global minimizer $U_{j}$ of $\mathcal{E}(u)$, $\mathcal{E}\left(U_{j}\right) \leq \ell^{*}+\epsilon_{j}$. We have $L_{j}$ uniformly bounded from below and above (Lemma 3.3) so we choose a convergent subsequence $L_{j} \rightarrow L$; functions $v^{j}(x)=U_{j}\left(x L_{j}\right)$ uniformly bounded in $H_{1}([0,1])$ since $\mathcal{E}\left(U_{j}\right)$ are bounded; $v^{j}$ are solutions of rescaled (1.2) :

$$
L_{j}^{-2} \partial_{x}^{2} v^{j}-F^{\prime}\left(v^{j}\right)=0,0 \leq x \leq 1 .
$$

Therefore they are bounded in $\mathcal{C}^{2}([0,1])$. Passing to the limit as $\epsilon_{j} \rightarrow 0$ over a subsequence we obtain in the limit a periodic solution $v \in H_{1}([0,1])$. Such a solution belongs to $\mathcal{C}^{2}([0,1])$. Therefore we obtain $U(x)=v(x / L)$, $\mathcal{E}(U)=\mathcal{J}(U)=\ell^{*}$. So we have obtained a smooth solution $U$ of (1.2) which is a global minimizer of Jacobian length from the homotopy class $\Xi_{1}^{0}$, $\mathcal{J}(U)=\ell^{*}$; it represents a smooth simple Jordan curve. Equation (1.3) holds for $U$ since it holds for $U_{j}$. The curve $U(x)$ has no self-intersections 
since by cutting off the extra cycle at a self-intersection we can make $\mathcal{J}(U)$ smaller.

The derivative $\partial_{x} U(x) \neq 0$ by (1.3) and (2.1). So the curve $C_{i}=$ $\operatorname{graph}(U)$ is smooth. The curve may be non-unique, so we take such that the area bounded by the curve is minimal. Clearly, the minimum is attained since the area is a continuous function of $u \in H_{1}([0, L])$ and $L$ and the set of considered solutions is compact in $H_{1}([0, L])$.

Now we prove that the $U(x)$ defined above determines a minimal cycle.

Consider for a given point $Q$ inside $C_{i}$ with $\operatorname{dist}\left(Q, C_{i}\right) \geq \eta^{\prime}$ all curves $\Gamma$ from $\Xi_{1}^{0}$ which pass through $Q$. Let $\gamma(Q)$ be the infinum of $\mathcal{J}(\Gamma)$ over such $\Gamma$. It is attained on a solution $U^{*}(x)$ of $(1.2), U^{*}(0)=U^{*}(L)=Q, U^{*} \in \widetilde{E}$, $\mathcal{J}\left(U^{*}\right)=\gamma(Q)$. One deduces it as above considering the same invariant set $\widetilde{E}$ of functions as before but subjected to an extra condition $u(0)=u(L)=Q$. This condition corresponds to the case of Dirichlet boundary conditions in Theorem 2.2. The infinum $\gamma(Q)$ is strictly greater than $\mathcal{J}\left(C_{i}\right)$. To show this, assume the contrary, $\gamma(Q)=\mathcal{J}\left(C_{i}\right)$. First, the curve $U^{*}$ cannot have common points with $C_{i}$.

Indeed, every two arcs with common endpoints of any two geodesic cycles which are global minimizers from $\Xi_{1}^{0}$ have the same Jacobian length (otherwise we could decrease the lengths of a cycle by interchanging the arcs). If the lengths are equal, interchanging the arcs we again obtain minimal geodesics from $\Xi_{1}^{0}$. The curves obtained by interchanging may have corner points. Since every minimal geodesic is invariant under the parabolic flow for some $L$ and the flow decreases lengths of non-equilibria, the minimizer so obtained should be a smooth curve. Therefore the curves at points of intersection have to be tangent. They are solutions of (1.2) with the same value of $u$ at $x=x_{0}$; thanks to the tangency they have collinear values of $\partial_{x} u\left(x_{0}\right)$; Hamiltonian conservation (1.3) implies that $\left|\partial_{x} u\left(x_{0}\right)\right|$ is the same. Their orientation is the same. Therefore solutions of (1.2) coincide. If the curves have only one common point they again are tangent and coincide. So the only possibility left is a curve $U^{*}$ which does not intersect $U$ at all. But in this case it lies inside $C_{i}$ and bounds area smaller than $C_{i}$ which contradicts the definition of $C_{i}$. So $\gamma(Q) \geq \mathcal{J}(U)+\epsilon_{2}$, with $\epsilon_{2}^{\prime}>0$ depending on $Q$; so $C_{i}$ is weakly minimal. Now we consider $\epsilon_{2}=\inf \left(\epsilon_{2}^{\prime}(Q)\right)$ with the infinum over $Q$ from the closed set $Y=\left\{\operatorname{dist}\left(Q, C_{i}\right)=\eta^{\prime}\right\}$; we have $\epsilon_{2}>0$. We prove this from the contrary as before, assuming that $\epsilon_{2}=0$ and choosing a convergent sequence $U^{*}\left(Q_{j}\right)$ of the above solutions with $Q_{j} \rightarrow Q_{0} \in Y$, $\epsilon_{2}^{\prime} \rightarrow 0$. We get $\mathcal{J}\left(U\left(Q_{j}\right)\right) \rightarrow \mathcal{J}\left(U\left(Q_{0}\right)\right)=\mathcal{J}\left(C_{i}\right)$, but this contradicts the weak minimality. So $C_{i}$ is minimal. 
The cycle $C_{i}$ is $\theta$-stable with respect to $C_{i}^{1}$. Note first that if there is a cycle $\Gamma_{0}$ in the band between $C^{3}$ and $C_{i}^{1}, \mathcal{J}\left(\Gamma_{0}\right) \geq \mathcal{J}\left(C_{i}\right)$ by construction of $C_{i}$ as a global minimizer. After that we apply the proof of Lemma 3.1 to the case when a general curve $\Gamma$ has a point on $C_{i}^{1}$, hence one of the cycles formed by self-intersections has length not smaller than $\mathcal{J}\left(C_{i}\right)+\theta$, and all other have Jacobian lengths which are not smaller than $\mathcal{J}\left(C_{i}\right)$. This implies the $\theta$-stability.

Remark 3.2. In the situation of Example 3.2 if $F(u) \leq m$ when $\left|u-P_{1}\right|=$ $R, R>r_{2}$, a sufficient condition for existence of a minimal cycle which follows from Theorem 3.2 is

$$
\sqrt{M} r_{2} /(\pi+1)>R \sqrt{m} .
$$

In this case the cycle $C_{i}^{1}$ is given by $\left|u-P_{i}\right|=r_{2} /(\pi+1)$.

If we have several bands with large sizes and encircling them cycles with small Jacobian lengths, we obtain existence of several non-intersecting minimal cycles.

Theorem 3.3. Let $D_{i}^{1}$ be encircled by bands $B_{i}$ (bounded by $C_{i}^{1}$ and $C_{i}^{2}$ such that $D_{i}^{2} \cap D_{k}^{2}=\emptyset$ for $\left.k \neq i\right)$ with size not less than $\beta_{i}$. Let cycles $C_{i}^{3}=\partial D_{i}^{3}$ satisfy $D_{i}^{1} \subset D_{i}^{2} \subset D_{i}^{3}$ and $\mathcal{J}\left(C_{i}^{3}\right)<2 \beta_{i}$, and assume that $D_{k}^{2} \cap D_{i}^{3}=\emptyset$ for $k \neq i$. Then there exist minimal cycles $C_{i}$ encircling $D_{i} \subset D_{i}^{3}$. These cycles are $\theta$-stable with respect to $C_{i}^{1}$ if $\theta<2 \beta_{i}-\mathcal{J}\left(C_{i}^{3}\right)$ for $i=1, \ldots, n$. The domains $D_{i}$ do not contain points of cycles $C_{k}^{1}, k \neq i$; moreover, $C_{k} \cap C_{i}=\emptyset$ for $k \neq i$.

Proof. The proof is similar to the proof of Theorem 3.2. Now we minimize Jacobian length in the class of curves which have homotopy type $g_{i}^{1}$ in $\mathbf{R}^{2} \backslash$ $\cup_{1}^{n} D_{k}^{1}$ and not in $\mathbf{R}^{2} \backslash D_{i}^{1}$ with one fixed $i$ as we did in Theorem 3.2. We take in the proof $\epsilon_{j}<\theta / 2$, so a quasi-minimizing curve $u$ in $\mathbf{R}^{2} \backslash \cup_{1}^{n} D_{k}^{1}$ with $\mathcal{J}(u) \leq \ell^{*}+\epsilon_{j} \leq \ell^{*}+\theta / 2$ does not intersect with $C_{k}^{1}, k \neq i$. Indeed, if a piece of the curve $u(s)$ intersects $C_{k}^{1}$ that is $u\left(s_{*}\right) \in C_{k}^{1}$, it has to intersect $C_{k}^{3}$ (otherwise it would lie in $D_{k}^{3}$ and its homotopy type can't be $g_{i}^{1}$ since $D_{k}^{3}$ is contractible in $\left.\mathbf{R}^{2} \backslash D_{i}^{1}\right)$. Therefore $u(s) \in D_{k}^{3}$ for $s_{1}<s<s_{2}$, $u\left(s_{1}\right) \in C_{k}^{3}, u\left(s_{2}\right) \in C_{k}^{3}$. It has two arcs connecting $C_{k}^{3}$ with $C_{k}^{1}$; these arcs intersect $C_{k}^{2}$, therefore their Jacobian length is not less than $\beta_{k}$. Replacing the piece of the curve on $\left[s_{1}, s_{2}\right]$ by the arc of $C_{k}^{3}$ between points $u\left(s_{1}\right) \in u\left(s_{2}\right)$ we decrease the Jacobian length at least by $2 \beta_{k}-\mathcal{J}\left(C_{k}^{3}\right)>\theta$ and the 
resulting length is less then $\ell^{*}(b)$. Since the replacement can be included into a homotopy in the ring between $C_{k}^{3}$ and $C_{k}^{1}$, the homotopy type of this curve is again $b=g_{i}^{1}$ and we obtain a contradiction with definition of $\ell^{*}(b)$. Proceeding as in Theorem 3.2 we obtain a minimizer which gives a minimal cycle $C_{i}$ for every $i$. The cycle $C_{i}$ does not intersect $C_{k}^{1}, k \neq i$ and is $\theta$-stable with respect to $C_{i}^{1}$. If two minimal cycles $C_{k}$ and $C_{i}$ intersect, we see as before that the arcs between intersection points should have equal lengths; but this contradicts minimality. Like in the proof of Theorem 3.2 we show that tangency is also impossible.

Remark 3.3. We may consider a plane as a sphere with a deleted point at infinity, and take a neighborhood $D_{0}^{\prime}=\| R^{2} \backslash \bar{D}_{0}$ of infinity as exterior of a cycle $C_{0}$; we also take another neighborhood $D_{0}^{1 \prime}$ of infinity which is bounded by a cycle $C_{0}^{1} \subset D_{0}^{\prime}$. Below we show that minimal cycles $C_{i}, i=1, . ., n$ exist inside the external minimal cycle $C_{0}$. This observation allows to relax condition $F(u) \geq \mu$ for all $u$, see below for details.

Theorem 3.4. Let $D_{0}^{3} \subset D_{0}^{2} \subset D_{0}^{1}$. Let an external band $B_{0}$ with size $\beta_{0}$ be bounded by cycles $C_{0}^{1}, C_{0}^{2}\left(C_{0}^{1}\right.$ outside $\left.C_{0}^{2}\right)$. Let $F(u) \geq \mu>0 \forall u \in D_{0}^{1}$, that is inside $C_{0}^{1}$. Let bands $B_{i}, i=1, \ldots, n$ with sizes $\beta_{i}$ be encircled by cycles $C_{i}^{3}$ and all $C_{i}^{3}, i=1, \ldots, n$ be inside $D_{0}^{3}$.

Let $\mathcal{J}\left(C_{i}^{3}\right)<2 \beta_{i}, i=0,1, \ldots, n$. Then there exist minimal cycles $C_{i}, i=$ $1, \ldots, n$ such that the domains $D_{i}, i=1, \ldots, n$ encircled by them do not intersect and they lie inside an external minimal cycle $C_{0}$ which lies inside $C_{0}^{1}$. The cycles $C_{i}, i=0,1, \ldots, n$ are $\theta$-stable with $\theta=\min _{i=0, \ldots, n}\left(2 \beta_{i}-\mathcal{J}\left(C_{i}^{3}\right)\right)$ with respect to $C_{i}^{1}$.

Proof. The proof is similar to the proofs of Theorems 3.2 and 3.3. First we extend $F(u) \geq \mu$ outside $C_{0}^{1}$. Then we prove existence of minimal nonintersecting cycles $C_{i}, i=0,1, \ldots, n$ like in Theorem 3.3; the cycles are $\theta$ stable with respect to $C_{i}^{1}$. Since all cycles constructed are inside $C_{0}^{1}$, the values of $F(u)$ outside $C_{0}^{1}$ do not influence the minimality and $\theta$-stability properties of all $C_{i}, i=0, \ldots, n$.

Remark 3.4. If $F(u) \geq M=M\left(R_{1}\right)$ when $|u| \geq R_{1}$ we can take as $C_{0}^{3}$ the circle $|u|=R_{0}$ where $R_{0}$ is large enough to include all $C_{i}$ and is fixed. We take the band $B_{0}=\left\{R_{1} \leq|u| \leq(\pi+1) R_{1}\right\}$. Then the size of the external band is not less than $\sqrt{2 M} \pi R_{1}$; if $R_{1}^{2} M\left(R_{1}\right) \rightarrow \infty$ when $R_{1} \rightarrow \infty$ the size becomes arbitrarily large when $R_{1}$ is large. 
Infinitely spatially complex solutions of PDE

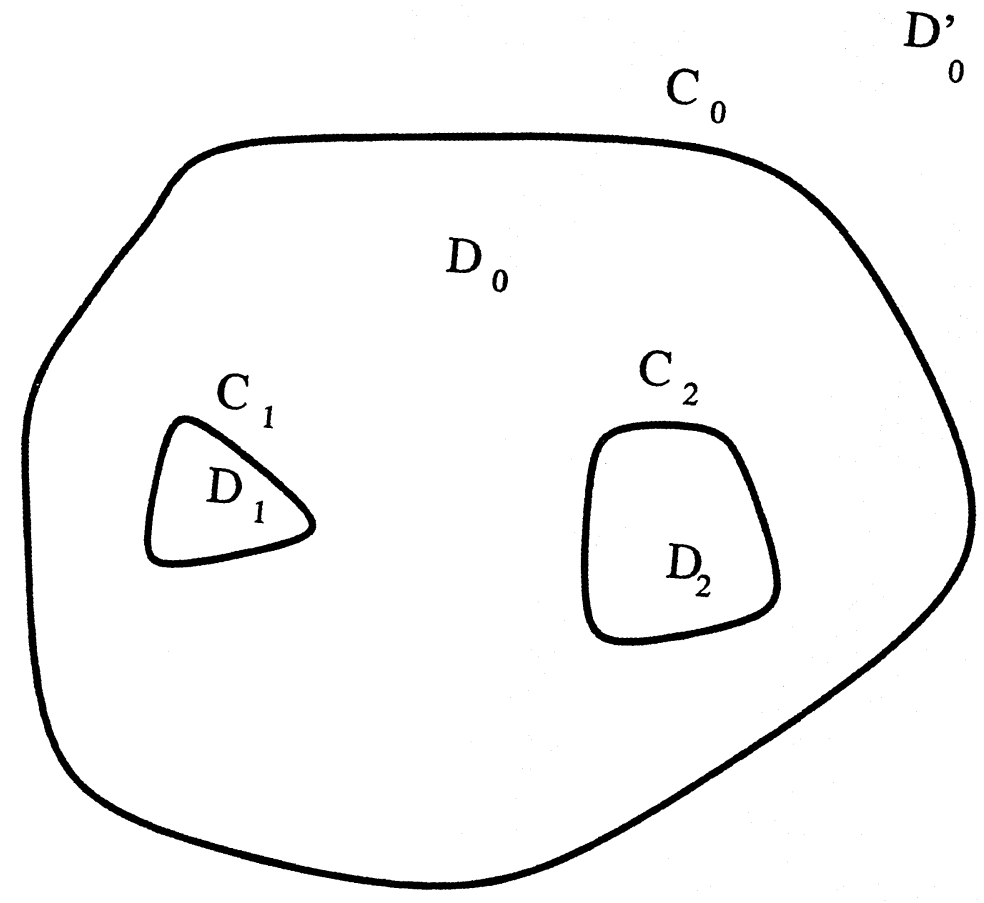

Figure 3.

Remark 3.5. If $n=1 C_{0}$ may coincide with $C_{1}$. In this case Theorem 3.4
gives $\theta$-stability of $C_{1}$ with respect to $C_{1}^{1}$ and $C_{0}^{1}$. Everywhere below fixed external cycle $C_{0}^{1}$. We fix domain $\geq \mu \forall u$ means for all $u$ inside a We modify now definitions 3.3 . of Theorem 3.1 holds for so modified 3.4 of sets $\Xi$ and $\Xi^{\prime}$. The statement

Definition 3.6. We denote by $\Xi$ the set of closed curves $u(s)$ which do not $\Xi_{b}$ the set of curves from $\Xi$ which belong to $b$ and put $b \in \pi_{1}^{\prime}\left(D_{0}^{1} \backslash \cup_{i} D_{i}^{1}\right)$ by

$$
\ell^{*}(b)=\inf _{u \in \Xi_{b}} \mathcal{J}(u)
$$


Definition 3.7. We denote by $\Xi^{\prime}([0, L])$ and $\Xi_{b}^{\prime}([0, L])$ the sets of functions $u \in H_{1}([0, L])$ which have graphs from $\Xi$ and $\Xi_{b}$ respectively.

The sufficient conditions for existence of minimal cycles we give above imply that if a potential $F_{0}(u)$ has a critical point $P_{0}$, then there exists a perturbation of this potential, which has an arbitrarily small $\mathcal{C}^{2-\epsilon}$-norm, such that the perturbed potential has arbitrarily many minimal cycles near the point $P_{0}$. This shows that existence of many minimal cycles is typical. Note that in the following theorem we discuss properties of the potential $F_{0}(u)$ only near the point $P_{0}$. We also do not assume that $F_{0}(u)$ is positive as we do everywhere else.

Theorem 3.5. Let $n=m^{2}$ with an integer $m$. Let $\left|F_{0}(u)-F_{0}\left(P_{0}\right)\right| \leq$ $K_{0}\left|u-P_{0}\right|^{q_{0}}, q_{0}>1$ when $\left|u-P_{0}\right| \leq \delta_{0} ;$ let $\mu=\rho_{0}^{q_{0}} K_{0}$. Then for sufficiently small $\rho_{0}>0$ there exists a function $\Psi(u) \geq 0, \Psi(u)=0$ when $\left|u-P_{0}\right| \geq \rho_{0}$ and

$$
\|\Psi\|_{\mathcal{C}^{\gamma}} \leq K^{\prime} \rho_{0}^{q_{0}-\gamma} n^{\gamma / 2}
$$

which has the following property. The Jacobian metric corresponding to the potential $\widetilde{F}(u)=F_{0}(u)-F_{0}\left(P_{0}\right)+\Psi(u)+\mu$ has $n$ minimal cycles contained in an external minimal cycle which is inside the domain $\left|u-P_{0}\right| \leq \rho_{0}$.

Proof. We take $F_{0}\left(P_{0}\right)=0$ for brevity. First, the potential $F(u)=F_{0}(u)+$ $\mu>0$ if $\eta=\left|u-P_{0}\right|<\rho_{0}=\left(\mu / K_{0}\right)^{1 / q_{0}}$. Let $\Psi_{0}(\eta)$ be a smooth non-negative function, $\Psi_{0}(\eta)=0$ when $|\eta| \leq 1 / 4, \Psi_{0}(\eta) \geq 1$ when $1 / 2 \leq|\eta| \leq 3 / 4$. Let $\Psi_{1}(\eta)$ be a smooth non-negative function, $\Psi_{1}(\eta)=0$ when $|\eta| \geq 1, \Psi_{1}(\eta) \geq 1$ when $|\eta| \leq 1 / 2$. Let $m \geq 1$ be an integer, $\rho_{1}=\rho_{0} /(8 m)$ and $P_{i}, i=$ $1, \ldots, n, n=m^{2}$ be points in $D_{0}=\left\{\left|u-P_{0}\right|<\rho_{0} / 4\right\}$ such that their $\rho_{1-}$ neighborhoods do not intersect and lie in $D_{0}$. It is always possible to find $n$ such points. Now we put

$$
\tilde{F}(u)=F_{0}(u)+M_{0} \Psi_{0}\left(\left|u-P_{0}\right| / \rho_{0}\right)+\mu+M_{1} \sum_{i} \Psi_{1}\left(\left|u-P_{i}\right| / \rho_{1}\right) .
$$

The external band $B_{0}=\left\{1 / 2 \leq\left|u-P_{0}\right| / \rho_{0} \leq 3 / 4\right\}$ has size not less than $\sqrt{2 M_{0}} \rho_{0} / 4$. The cycle $C_{0}^{3}=\left\{\left|u-P_{0}\right|=\rho_{0} / 4\right\}$ has $\mathcal{J}\left(C_{0}^{3}\right)=\pi \sqrt{2 \mu} \rho_{0} / 2$. We take $M_{0}=4 \pi^{2} \mu$. The internal bands $B_{i}=\left\{1 / 4 \leq\left|u-P_{i}\right| / \rho_{1} \leq 1 / 2\right\}$ have size not less than $\sqrt{2 M_{1}} \rho_{1} / 4$. The cycle $C_{i}^{3}=\left\{\left|u-P_{i}\right|=\rho_{1}\right\}$ has $\mathcal{J}\left(C_{i}^{3}\right) \leq 2 \pi \sqrt{2 \mu} \rho_{1}$. We take $M_{1}=32 \pi^{2} \mu$. So conditions of Theorem 3.4 are fulfilled and there exist $n$ minimal cycles inside the external minimal 
cycle $C_{0}$. Now we estimate the norm of the perturbation. We have an estimate of the Hölder seminorm $\|\cdot\|^{\prime}$ of order $\gamma$ (or derivatives of order $\gamma$ )

$$
\left\|M_{1} \Psi_{1}\left(\left|u-P_{i}\right| / \rho_{1}\right)\right\|_{\mathcal{C}^{\gamma}}^{\prime} \leq c M_{1} / \rho_{1}^{\gamma} \leq c_{1} m^{\gamma} \rho_{0}^{q_{0}-\gamma} .
$$

Estimating the $\mathcal{C}$-norm we obtain

$$
\left\|M_{1} \Psi_{1}\left(\left|u-P_{i}\right| / \rho_{1}\right)\right\|_{\mathcal{C}} \leq c_{3} M_{1} \leq c_{4} \rho_{0}^{q_{0}} .
$$

Similar estimates hold for $M_{0} \Psi_{0}$. Since we can take $\rho_{0}$ arbitrary small, these inequalities imply the assertion of Theorem 3.5.

Remark 3.6. In the scalar case stable constant solutions $u=c_{i}$ with $f^{\prime}\left(c_{i}\right)=0$ of the equation $\partial_{x}^{2} u-f^{\prime}(u)=0$ with periodic boundary conditions create barriers for dynamics of the parabolic equation $\partial_{t} u=\partial_{x}^{2} u-f^{\prime}(u)$; the set $c_{1} \leq u(x) \leq c_{2}$ is invariant thanks to the Maximum Principle. Minimal cycles are rather a soft obstacle, they do not create a non-penetrable barrier for dynamics of the parabolic PDE. One can easily see this by writing (1.1) in polar coordinates centered at $P_{1}$ in the situation of Example 3.1 :

$$
\begin{gathered}
\partial_{t} \eta=\partial_{x}^{2} \eta-\eta\left(\partial_{x} \phi\right)^{2}-f^{\prime}(\eta), \\
\eta \partial_{t} \phi=\eta \partial_{x}^{2} \phi+2 \partial_{x} \eta \partial_{x} \phi .
\end{gathered}
$$

Let $\eta=\eta_{0}=$ const where $\eta_{0}$ satisfies (3.7) (in particular $f^{\prime}\left(\eta_{0}\right) \eta_{0}+2 f\left(\eta_{0}\right)=$ $0)$, let $\partial_{x} \phi=\phi^{\prime}=$ const where the constant $\phi^{\prime}$ is determined from (1.3) which takes the form

$$
\left(\partial_{x} \eta\right)^{2}+\eta^{2}\left(\partial_{x} \phi\right)^{2}=2 f\left(r_{i}\right)
$$

and let $L=2 \pi / \phi^{\prime}$. One can easily check that we obtain a steady-state solution of (3.13), (3.14) which determines by Lemma 3.2 a minimal cycle. If we take initial data $\eta(x, 0), \phi(x, 0)$ such that $\eta(x, 0)=\eta_{0}=$ const is the same and $\phi(x, 0)$ perturbed so that $\left|\partial_{x} \phi\left(x_{0}, 0\right)\right|^{2}>-f^{\prime}\left(\eta_{0}\right) / \eta_{0}$ at a point $x_{0}$, than from (3.13) we obtain $\partial_{t} \eta\left(x_{0}, 0\right)<0$ so $\eta\left(x_{0}, t\right)<\eta_{0}$ for small $t>0$, and the curve $u(x, t)$ gets inside the minimal cycle at $x=x_{0}$. Nevertheless, if the energy of perturbation is small, the curve $u(x, t)$ gets only a small distance inside the minimal cycle according to Theorem 3.1 and Remark 3.1 . 


\section{Steady-state solutions of a given type.}

From now on we assume that there exist $n+1$ minimal cycles $C_{i}, i=0,1, \ldots, n$ which do not intersect, which are boundaries of domains $D_{i}$ and which are $\theta$-stable with respect to $C_{i}^{1}=\partial D_{i}^{1}, i=1, \ldots, n$ with a fixed $\theta$. The open non-intersecting domains $D_{i}, D_{i}^{1}, i=1, \ldots, n$ have properties described in the previous section; moreover, they lie inside the domain $D_{0}$ bounded by the external minimal cycle $C_{0}$. We denote $D^{\prime}=D_{0}^{1} \backslash \cup_{i=1}^{n} \bar{D}_{i}^{1}, D^{\prime \prime}=D_{0} \backslash \cup_{i=1}^{n} \bar{D}_{i}$.

We recall that the fundamental homotopy group $\pi_{1}\left(D^{\prime}, \sigma\right)$, where $\sigma \in \bar{D}^{\prime}$ is a contractible set, is the set of classes of homotopy equivalent mappings $u$ from the segment $[0,1]$ into $\bar{D}^{\prime}$ such that $u(0) \in \sigma, u(1) \in \sigma$; there is a standard group structure on this set. The set of mappings depends on $\sigma$, but since $\sigma$ is contractible and $\bar{D}^{\prime}$ is connected, there is a natural isomorphism between the sets of classes of equivalence, so we identify $\pi_{1}\left(D^{\prime}, \sigma\right)$ with different $\sigma$. Below we fix a point $P_{*} \in D^{\prime \prime}$ and take a contractible $\sigma$ which contains $P_{*}$. The contraction of $\sigma$ into $P_{*}$ takes curves with endpoints in $\sigma$ into curves with endpoints in $P_{*}$ which gives the isomorphism of $\pi_{1}\left(D^{\prime}, P_{*}\right)$ and $\pi_{1}\left(D^{\prime}, \sigma\right)$. So we identify $\pi_{1}\left(D^{\prime}, P_{*}\right)=\pi_{1}\left(D^{\prime}, \sigma\right)$. (For more detail see, for example, [12], [20], [26].) We denote for brevity $\pi_{1}\left(D^{\prime}, P_{*}\right)=\pi_{1}$.

The homotopy group $\pi_{1}$ of the disc with $n$ holes $D^{\prime}$ is a free group with $n$ generators $g_{i}$. Every $g_{i}$ corresponds to a counterclockwise turn around $D_{i}$. A pair of arbitrary finite sequences of non-zero integers $k_{1}, \ldots, k_{N}$ and $i_{1}, . . i_{N}$ with $i_{j+1} \neq i_{j}$ where $i_{j} \in\{1, \ldots, n\}$ determine an irreducible word

$$
b=g_{i_{1}}^{k_{1}} g_{i_{2}}^{k_{2}} \ldots g_{i_{N}}^{k_{N}} .
$$

The fundamental group $\pi_{1}$ consists of all irreducible words and of the unit element 1 which corresponds to the trivial class. The sum $\left|k_{1}\right|+\cdots+\left|k_{N}\right|=$ $\operatorname{deg} b$ is called the degree of the word $b, \operatorname{deg}(1)=0$. Obviously, $b$ can be represented as a product of deg $b$ elements $g_{1}, g_{2}, g_{1}^{-1}, g_{2}^{-1}$ etc. The group multiplication in $\pi_{1}$ includes the group relations $g_{i} g_{i}^{-1}=1$ and $g_{i}^{-1} g_{i}=1$.

The set of all possible words $w$ with letters $g_{i}, g_{i}^{-1}$ without the group relations is denoted by $\tilde{\pi}_{1}$. The number of letters in a word is called the degree of the word. Two words $w_{1}, w_{2} \in \tilde{\pi}_{1}$ are equivalent: $w_{1} \sim w_{2}$ if they determine the same element $b \in \pi_{1}$ after cancellations of pairs $g_{i} g_{i}^{-1}$ or $g_{i}^{-1} g_{i}$.

The homotopy class $h(u) \in \pi_{1}$ is defined for a curve which lies in $\bar{D}^{\prime}$ and has endpoints in $\sigma$, such curves are graphs of functions on an interval $[0, L]$ which have boundary values $u(0), u(L)$ in $\sigma$; such functions arise as solutions of Dirichlet boundary problems. 
Homotopy classes of closed curves in $D^{\prime}$ correspond to functions satisfying periodic boundary conditions. The set of these homotopy classes (we denote it by $\pi_{1}^{\prime}$ ) can be described as the set of words from $\pi_{1}$ with an additional equivalence relation: a word $b^{\prime}$ is equivalent to the word $b_{1}^{\prime}$ if the first word is a cyclic permutation of the second.

We give a theorem of existence of a periodic solution with an arbitrary homotopy type.

Theorem 4.1. For every nontrivial homotopy class $b \in \pi_{1}^{\prime}$ there exists $L$ and a steady-state $L$-periodic solution $U$ of the equation (1.2) such that $U \in$ $b$,

$$
\mathcal{E}(U)=\mathcal{J}(U)=\ell^{*}(b),
$$

and the Hamiltonian conservation law (1.3) holds. The graph of the solution either coincides with one of $C_{i}$ or lies in $D_{0}$ and does not intersect $C_{i}, i=$ $0, \ldots, n$, that is it lies in $D^{\prime \prime}$. If the function $F(u)$ is twice continuously differentiable, then this solution is stable in the Lebesgue space $\mathcal{L}_{2}$, namely

$$
\int_{0}^{L}\left[\partial_{x}^{2} V(x)-F^{\prime \prime}(U) V(x)\right] V(x) d x \leq 0
$$

for any smooth L-periodic function $V(x)$.

Proof. The cycles $C_{i}$ are $\theta$-stable with a fixed $\theta>0$. We take a sequence of smooth curves $u_{j}(s) \in b$ in $D^{\prime}$ such that $\mathcal{J}\left(u_{j}\right) \leq \ell^{*}(b)+\epsilon_{j}, \epsilon_{j} \leq \theta / 2, \epsilon_{j} \rightarrow 0$. Using the parametrization from Lemma 3.3 we find functions $u_{j}(x), 0 \leq x \leq$ $L_{j}, \mathcal{J}\left(u_{j}\right)=\mathcal{E}\left(u_{j}\right)$; corresponding lengths (see Lemma 3.3) of $x$-intervals are denoted by $L_{j}$. We take $u_{j}(x)=u_{j}(x, 0)$ as initial data for the parabolic equation (1.1) with the spatial period $L_{j}$. We obtain using (2.5) that

$$
\mathcal{J}\left(u_{j}(t)\right) \leq \mathcal{E}\left(u_{j}(t)\right) \leq \mathcal{E}\left(u_{j}(0)\right)=\mathcal{J}\left(u_{j}(0)\right) \leq \ell^{*}(b)+\epsilon_{j} .
$$

The set $\left\{\mathcal{E}(u) \leq \ell^{*}(b)+\epsilon_{j}\right\} \cap \Xi_{b}^{\prime}\left(\left[0, L_{j}\right]\right)$ is invariant by Theorem 3.1. Boundedness of curves in $D_{0}$ implies boundedness of the sup-norm. By Theorem 2.2 we obtain existence for every $j$ of a stable solution $U_{j}$ of (1.2), which provides the absolute minimum of energy over the class $\Xi_{b}^{\prime}\left(\left[0, L_{j}\right]\right), \mathcal{E}\left(U_{j}\right) \leq \ell^{*}(b)+\epsilon_{j}$. The curve $U_{j}$ does not intersect the cycles $C_{i}^{1}$ (see the proof of Theorem 3.2). Therefore $U_{j}$ is in the interior of $\left\{\mathcal{E}(u) \leq \ell^{*}(b)+\epsilon_{j}\right\} \cap \Xi_{b}^{\prime}\left(\left[0, L_{j}\right]\right)$. Since $U_{j}$ is a minimizer of $\mathcal{E}$, its second differential at $U_{j}$ is non-negative, hence $U_{j}$ is stable. 
Now we tend $j$ to infinity and $\epsilon_{j}$ to zero. By Lemma $3.3 L_{j}$ are uniformly bounded from above and below, so we choose a subsequence $L_{j} \rightarrow L>0$.

We have $\ell^{*}(b)+\epsilon_{j} \geq \mathcal{E}\left(u_{j}\right) \geq \ell^{*}(b)$, therefore $\mathcal{E}\left(u_{j}\right) \rightarrow \ell^{*}(b)$ as $j \rightarrow \infty$. Absolute values $\left|U_{j}(x)\right|$ are bounded since their graphs $U_{j}(s)$ are in the bounded domain $D_{0}$. Functions $\mathcal{U}_{j}(x)=U_{j}\left(L_{j} x / L\right)$ are $L$-periodic solutions of

$$
\left(L / L_{j}\right)^{2} \partial_{x}^{2} \mathcal{U}-F^{\prime}(\mathcal{U})=0 .
$$

Therefore $\mathcal{U}_{j}(x)$ are bounded in $\mathcal{C}^{2}$. Since all $\mathcal{U}_{j}$ are bounded in $H_{2}$, we easily construct convergent sequences $\mathcal{U}_{j}, \mathcal{U}_{j} \rightarrow \mathcal{U}$ to a solution $\mathcal{U}$ strongly in $H_{1}, \mathcal{E}\left(\mathcal{U}_{j}\right) \rightarrow \ell^{*}(b)$, so $\mathcal{E}(\mathcal{U})=\ell^{*}(b)$. Since $\ell^{*}(b) \leq \mathcal{E}(\mathcal{U}) \leq \mathcal{J}(\mathcal{U}) \leq \ell^{*}(b)$ we have $\mathcal{E}(\mathcal{U})=\mathcal{J}(\mathcal{U})$, therefore we obtain the minimizer of $\mathcal{J}$ as well as of $\mathcal{E}$, equation (1.3) holds by (3.1). Like in the proof of Theorem 3.3 we show that if the graph of $U$ intersects with $C_{i}$, it should coincide with it.

For a curve $u(s), s \in[0,1]$ which lies in $D^{\prime}$ and intersects the set $\sigma$, $u\left(s_{1}\right)=p_{1}, u\left(s_{2}\right)=p_{2}$ with $p_{1}, p_{2} \in \sigma$, we consider the restriction of $u(s)$ on $\left[s_{1}, s_{2}\right]$ and denote it by $\left.u\right|_{\left[s_{1}, s_{2}\right]}$. For this restriction the homotopy type $h\left(\left.u\right|_{\left[s_{1}, s_{2}\right]}\right) \in \pi_{1}$ is well defined.

Definition 4.4. We denote by $\Xi\left(p_{1}, p_{2}\right)$ the set of curves $u(s)$ in $\bar{D}^{\prime}$ with fixed endpoints $p_{1}, p_{2} \in \sigma$. We denote for $b \in \pi_{1}$ by $\Xi_{b}$ the set of curves from $\Xi$ which belong to the class $b$ and put

$$
\ell^{*}\left(b, p_{1}, p_{2}\right)=\inf _{u \in \Xi_{b}\left(p_{1}, p_{2}\right)} \mathcal{J}(u) .
$$

Definition 4.5. We denote $\Xi^{\prime}\left([0, L], p_{1}, p_{2}\right)$ and $\Xi_{b}^{\prime}\left([0, L], p_{1}, p_{2}\right)$ the set of functions $u \in H_{1}([0, L])$ which have graphs belonging to $\Xi\left(p_{1}, p_{2}\right)$ and $\Xi_{b}\left(p_{1}, p_{2}\right)$ respectively.

The functionals $\mathcal{E}$ and $\mathcal{J}$ are naturally defined on restrictions of functions to subintervals:

$$
\begin{aligned}
& \mathcal{J}\left(\left.U\right|_{\left[x_{1}, x_{2}\right]}\right)=\int_{x_{1}}^{x_{2}} \sqrt{2 F(U(x))}\left|\partial_{x} U(x)\right| d x, \\
& \mathcal{E}\left(\left.U\right|_{\left[x_{1}, x_{2}\right]}\right)=\int_{x_{1}}^{x_{2}}\left[F(U(x))+\left|\partial_{x} U(x)\right|^{2} / 2\right] d x .
\end{aligned}
$$

Proposition 4.1. Let $u(x), x_{0} \leq x \leq x_{3}$ be a curve in $\bar{D}^{\prime}, u\left(x_{0}\right)=$ $p_{1}, u\left(x_{3}\right)=p_{2}$ with $p_{1}, p_{2} \in \sigma$. Let $u(x)$ intersect $\sigma$ at two more points, $u\left(x_{1}\right)=p_{1}^{\prime}, u\left(x_{2}\right)=p_{2}^{\prime}$ with $p_{1}^{\prime}, p_{2}^{\prime} \in \sigma, x_{0} \leq x_{1} \leq x_{2} \leq x_{3}$. 
(i) If $\mathcal{J}(u) \leq \ell^{*}\left(b, p_{1}, p_{2}\right)+\zeta$ with $b=h\left(\left.u\right|_{\left[x_{0}, x_{3}\right]}\right), \zeta \geq 0$, then

$$
\ell^{*}\left(h\left(\left.u\right|_{\left[x_{1}, x_{2}\right]}\right), p_{1}^{\prime}, p_{2}^{\prime}\right) \leq \mathcal{J}\left(\left.u\right|_{\left[x_{1}, x_{2}\right]}\right) \leq \ell^{*}\left(h\left(\left.u\right|_{\left[x_{1}, x_{2}\right]}\right), p_{1}^{\prime}, p_{2}^{\prime}\right)+\zeta .
$$

(ii) If $\mathcal{E}(u) \leq \ell^{*}\left(b, p_{1}, p_{2}\right)+\zeta$, then

$$
\ell^{*}\left(h\left(\left.u\right|_{\left[x_{1}, x_{2}\right]}\right), p_{1}^{\prime}, p_{2}^{\prime}\right) \leq \mathcal{E}\left(\left.u\right|_{\left[x_{1}, x_{2}\right]}\right) \leq \ell^{*}\left(h\left(\left.u\right|_{\left[x_{1}, x_{2}\right]}\right), p_{1}^{\prime}, p_{2}^{\prime}\right)+\zeta
$$

(iii) Let deg $\left(h\left(\left.u\right|_{\left[x_{1}, x_{2}\right]}\right)\right) \leq d$. If $\mathcal{J}(u) \leq \ell^{*}\left(b, p_{1}, p_{2}\right)+\zeta$ with $b=$ $h\left(\left.u\right|_{\left[x_{0}, x_{3}\right]}\right)$, then $\mathcal{J}\left(\left.u\right|_{\left[x_{1}, x_{2}\right]}\right) \leq R_{d}$ where the constant $R_{d}$ does not depend on $u$.

Proof. The first inequality in (4.4) is trivial. If the second is not true, $\mathcal{J}\left(\left.u\right|_{\left[x_{1}, x_{2}\right]}\right)=\ell^{*}\left(h\left(\left.u\right|_{\left[x_{1}, x_{2}\right]}\right), p_{1}^{\prime}, p_{2}^{\prime}\right)+\zeta^{\prime}, \zeta^{\prime}>\zeta$. In this case we can replace a piece of $u(x)$ on $\left[x_{1}, x_{2}\right]$ by a curve with the same endpoints $p_{1}^{\prime}, p_{2}^{\prime}$, the same homotopy type and with the Jacobian length $\ell^{*}\left(h\left(\left.u\right|_{\left[x_{1}, x_{2}\right]}\right), p_{1}^{\prime}, p_{2}^{\prime}\right)+\epsilon$. Therefore the length is decreased by at least $\left.\zeta^{\prime}-\epsilon\right\rangle \zeta$ if $\epsilon$ is small; so we obtain a new curve $u^{\prime}$ with $h\left(u^{\prime}\right)=b, \mathcal{J}\left(u^{\prime}\right)<\ell^{*}\left(b, p_{1}, p_{2}\right)$ which contradicts the definition of $\ell^{*}$. Therefore (4.4) is true. To obtain (ii) we make a change of paramerization according to Lemma 3.3 so that $\mathcal{E}(u)=\mathcal{J}(u)$ on every interval; clearly $\ell^{*}$ does not depend on the parametrization.

To obtain (iii) note that for any given $b \in \pi_{1}$ we have a uniform bound $\ell^{*}\left(b, p_{1}, p_{2}\right) \leq \ell_{0}^{*}(b)$ for all $p_{1}, p_{2}$. Indeed, one can connect any two points $p_{1}, p_{2} \in \sigma$ by a contractible curve passing through $P_{*}$ and lying in a small neighborhood of $\sigma$ so that the length of the curve is uniformly bounded by a constant $K=K(\sigma)$. Then $\ell^{*}\left(b, p_{1}, p_{2}\right) \leq \ell^{*}\left(b, P_{*}, P_{*}\right)+K$. There is a finite number of classes $b^{\prime} \in \pi_{1}$ which satisfy $\operatorname{deg}\left(b^{\prime}\right) \leq d$, therefore corresponding $\ell^{*}\left(b, P_{*}, P_{*}\right)$ are bounded by a constant $R_{d}^{\prime}$. Using (i) we obtain that $\mathcal{J}\left(\left.u\right|_{\left[x_{1}, x_{2}\right]}\right) \leq R_{d}^{\prime}+\zeta=R_{d}$.

We obtain a simple corollary from Theorem 4.1:

Corollary 4.1. If the graph of the energy-minimizing solution $U(x)$ from Theorem 4.1 intersects a contractible closed set $\sigma \subset D^{\prime}$, that is for $x_{1}<x_{2}$ we have $u\left(x_{1}\right)=p_{1}^{\prime} \in \sigma, u\left(x_{2}\right)=p_{2}^{\prime} \in \sigma$ then

$$
\mathcal{J}\left(\left.U\right|_{\left[x_{1}, x_{2}\right]}\right)=\mathcal{E}\left(\left.U\right|_{\left[x_{1}, x_{2}\right]}\right)=\ell^{*}\left(h\left(\left.U\right|_{\left[x_{1}, x_{2}\right]}\right), p_{1}^{\prime}, p_{2}^{\prime}\right) .
$$


Proof. We can take any $x_{0}$ and $p_{1}=p_{3}=U\left(x_{0}\right)$; we can connect $p_{1}$ with $\sigma$ by a curve in $D^{\prime}$ and take the union of this curve and $\sigma$ as a new $\sigma$. After that we use Proposition 4.1 with $\zeta=0$.

Now we consider the Dirichlet problem (1.2), (2.3) with the boundary data $p_{1}=u(0), p_{2}=u(L)$ in $D^{\prime \prime}$. We take a contractible set $\sigma \subset \bar{D}^{\prime}$ and assume that $p_{1}, p_{2} \in \sigma \cap \bar{D}^{\prime \prime}$.

Theorem 4.2. Let $p_{1}, p_{2} \in \sigma \cap \bar{D}^{\prime \prime}$ Then for every $b \in \pi_{1}$ there exists $L$ and a steady-state solution of the equation (1.2), (2.3) such that $U \in b$,

$$
\mathcal{E}(U)=\mathcal{J}(U)=\ell^{*}\left(b, p_{1}, p_{2}\right)
$$

and (1.3) holds. If $F(u) \in \mathcal{C}^{2}\left(\mathbf{R}^{2}\right)$, the solution is stable, that is (4.3) holds for every smooth $V(x)$ with a compact support in $(0, L)$. If for $x_{1}<x_{2}$ we have $u\left(x_{1}\right)=p_{1}^{\prime} \in \sigma, u\left(x_{2}\right)=p_{2}^{\prime} \in \sigma$ then the equation (4.6) holds. The graph of $U$ lies in $\bar{D}^{\prime \prime}$.

Proof. The proof is completely similar to the proof of Theorems 4.1 and Corollary 4.1; now we obtain $U$ which is a global minimizer of $\mathcal{E}$ and $\mathcal{J}$ in $\Xi_{b}^{\prime}\left([0, L], p_{1}, p_{2}\right)$ and $\Xi_{b}\left(p_{1}, p_{2}\right)$, respectively. The only difference is that when $p_{1}, p_{2} \in C_{i}$ the graph of $U$ may intersect $C_{i}$ at $x=0, x=L$ not coinciding with $C_{i}$.

When the length of a curve $u$ is infinite, the functionals $\mathcal{E}(u)$ and $\mathcal{J}(u)$ are infinite and we cannot define stable geodesics of a prescribed type as their minima. To be able to pass to the limit as the length tends to infinity, we need to introduce localized characteristics which have limits. To this end we use a localization of the homotopy type. This localization depends on the set $\sigma$ and is defined below. Below we denote by $\widehat{\sigma}$ a contractible set in $\bar{D}^{\prime}$ which contains a $\delta$ - neighborhood of $\sigma$ in $\bar{D}^{\prime}$.

We consider a curve $u(s)$ in $\bar{D}^{\prime}$ which intersects $\sigma$. Let $u\left(s_{1}\right)=$ $p_{1}, u\left(s_{2}\right)=p_{2}$ with $p_{1}, p_{2} \in \sigma$. We denote the restriction of $u(s)$ on $\left[s_{1}, s_{2}\right]$ by $\left.u\right|_{\left[s_{1}, s_{2}\right]}$. The homotopy class of $\left.u\right|_{\left[s_{1}, s_{2}\right]}$ in $\pi_{1}$ is well-defined and we denote it by $h\left(\left.u\right|_{\left[s_{1}, s_{2}\right]}\right)$. If $u\left(s_{1}\right), u\left(s_{2}\right), u\left(s_{3}\right) \in \sigma$ we have the $\pi_{1}$-multiplication formula:

$$
h\left(\left.u\right|_{\left[s_{1}, s_{2}\right]}\right)=h\left(\left.u\right|_{\left[s_{1}, s_{2}\right]}\right) h\left(\left.u\right|_{\left[s_{2}, s_{3}\right]}\right) .
$$

For a curve $u(s)$ in $\bar{D}^{\prime}$ with endpoints in $\sigma$ for every $s_{*}$ such that $u\left(s_{*}\right) \notin$ $\sigma$ we can find a maximal open interval $\left(s_{1}, s_{2}\right)$ which contains $s_{*}$ and on 
which $u(s) \notin \sigma$. When the restriction onto $\left[s_{1}, s_{2}\right]$ has the trivial type, we call the interval non-essential. If $\left[s_{1}, s_{2}\right]$ is non-essential, then $h\left(\left.u\right|_{\left[s_{1}, s_{2}\right]}\right)=$ $h\left(\left.u\right|_{\left[s_{1}, s_{3}\right]}\right)$ when $s_{1}<s_{2}<s_{3}$. If a curve $u$ lies in $D^{\prime}$, has endpoints in $\sigma$ and belongs to a non-trivial class $b$, the Euclidean length $l_{E}(u)$ of this curve is bounded from below: $l_{E}(u) \geq l_{E}^{*}>0$. Indeed, if $l_{E}(u)<\delta$, the curve $u$ lies in a $\delta$-neighborhood of $\sigma$ and therefore in contractible $\widehat{\sigma}$, so it has a trivial type.

Therefore, if a curve $u$ has a finite length, there is only a finite number of essential intervals.

Definition 4.6. For a curve in $\bar{D}^{\prime}$ with endpoints in $\sigma$ and with a finite length we find all essential intervals $\left[s_{l}, s_{l}^{\prime}\right]$ and define the following word

$$
h_{\sigma}(u)=h\left(\left.u\right|_{\left[s_{1}, s_{1}^{\prime}\right]}\right) \ldots h\left(\left.u\right|_{\left[s_{N}, s_{N}^{\prime}\right]}\right)=\prod_{l=1}^{N} h\left(\left.u\right|_{\left[s_{l}, s_{l}^{\prime}\right]}\right) \in \tilde{\pi}_{1}
$$

where $N$ is the number of essential intervals, $s_{1}$ is the minimal of all left endpoints of essential intervals; $s_{N}^{\prime}$ is the maximal right endpoint. When there is no essential intervals at all, $N=0, h_{\sigma}(u)=1$ is trivial.

In a nontrivial (4.7) $s_{l}<s_{l}^{\prime} \leq s_{l+1}<s_{l+1}^{\prime},\left[s_{l}, s_{l}^{\prime}\right]$ are essential intervals of $u$ and $\left[s_{l}^{\prime}, s_{l+1}\right]$ are non-essential intervals for every $l$. It is possible that $s_{l}^{\prime}=s_{l+1}$.

Note that the reducible word $h_{\sigma}(u)$ is defined uniquely by $u$ as well as the essential intervals $\left[s_{l}, s_{l}^{\prime}\right]$; they depend on $\sigma$. Since the homotopy type does not depend on a contractible $\sigma$, irreducible words for two different $\sigma$ coincide: if $u$ has endpoints in $\sigma$ and $h(u)=b \in \pi_{1}$ and $\sigma \subset \sigma^{\prime}$, then we have

$$
h_{\sigma}(u) \sim h_{\sigma^{\prime}}(u) \sim b .
$$

Here and below $\sim$ denotes the equivalence of words by the group relation; two words are equivalent if they are related through cancellation or inserting a finite number of pairs $g_{i} g_{i}^{-1}$ or $g_{i}^{-1} g_{i}$. When two curves $u_{0}, u_{1}$ are connected by a homotopy in $\bar{D}^{\prime}$, we have $h\left(u_{0}\right)=h\left(u_{1}\right)$ but $h_{\sigma}\left(u_{0}\right)$ may be different from $h_{\sigma}\left(u_{1}\right)$; of course, $h_{\sigma}\left(u_{0}\right) \sim h_{\sigma}\left(u_{1}\right)$.

Below we define such sets $\sigma$ that $h_{\sigma}(u) \in \tilde{\pi}_{1}$ gives a detailed enough information on the localization of elements of $h(u) \in \pi_{1}$.

Let $P_{*} \in D^{\prime \prime}$ be a base point (we take it as the origin $O$ in the $u$-plane, $P_{*}=O$ ). We fix a spider-shaped set $\sigma^{*} \in \bar{D}^{\prime}$ (see Fig. 4) which consists of simple curves $\sigma_{i}, i=1, \ldots, n$ which start at the origin and connect it with the external cycle $C_{0}^{1}$. The curves intersect only at the origin; they intersect $C_{0}^{1}$ 
only once; therefore they divide the interior of $C_{0}^{1}$ into $n$ sectors $\Sigma_{i}$; every sector includes one of the minimal cycles $C_{i}$. The set $\sigma^{*}$ is contractible, we take it as a base point of the homotopy group $\pi_{1}=\pi_{1}\left(D^{\prime}, \sigma^{*}\right)$. We number the cycles $C_{i}$ so that the cycle $C_{i}$ is in the sector $\Sigma_{i}$ bounded by $\sigma_{i}$ and $\sigma_{i+1}$; clearly $\sigma_{n+1}=\sigma_{1}$. Consider now (4.7) for this set $\sigma=\sigma^{*}$. The function $u(s)$ on every essential interval takes values in one of $\Sigma_{i}$, so its type is $g_{i}^{\alpha}$ where $g_{i}$ is the generator corresponding to $C_{i} \subset \Sigma_{i}$. If the irreducible word $h\left(\left.u\right|_{\left[s_{1}, s_{1}^{\prime}\right]}\right) \in \pi_{1}$ of a restriction of $u(s)$ on $\left[s_{1}, s_{1}^{\prime}\right]$ includes more than one generator, there exists one more point of intersection with $\sigma^{*} u\left(s_{*}\right) \in \sigma^{*}$, $s_{1}<s_{*}<s_{1}^{\prime}$. Indeed, different $\Sigma_{i}$ cannot be connected by curves in $\bar{D}^{\prime}$ which do not intersect $\sigma^{*}$. So, if there is no point of intersection with $\sigma^{*}$, the curve $\left.u\right|_{\left[s_{1}, s_{1}^{\prime}\right]}$ lies in one of $\Sigma_{i}$ and has the type $g_{i}^{k}$. Therefore for every essential interval $h\left(\left.u\right|_{\left[s_{l}, s_{l}^{\prime}\right]}\right)=g_{i_{l}}^{\delta_{l}}$ and (4.7) takes the form

$$
h_{\sigma}(u)=g_{i_{1}}^{k_{1}} g_{i_{2}}^{k_{2}} \ldots g_{i_{n}}^{k_{n}}=\prod_{l=1}^{N} g_{i_{l}}^{k_{l}} \in \widetilde{\pi}_{1} .
$$

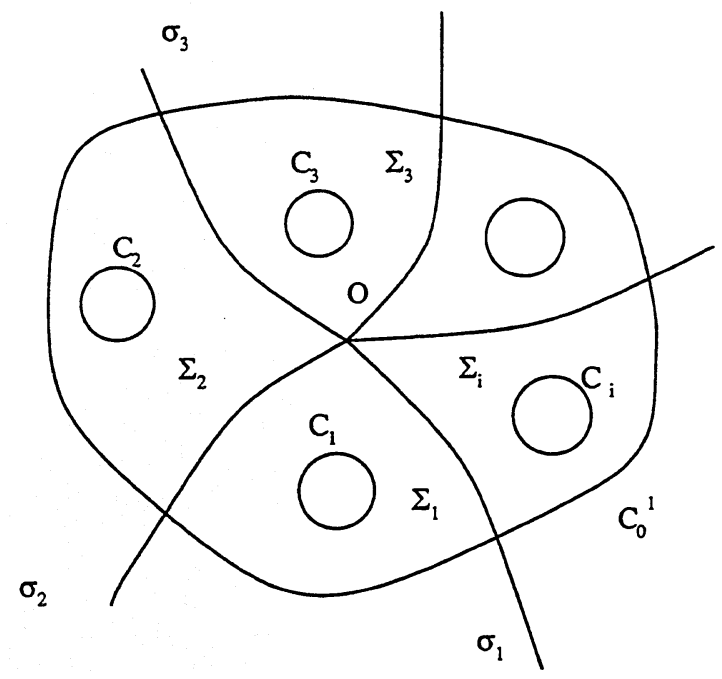

Figure 4: The set $\sigma$

Now we give another set $\sigma$ which gives more information, we denote this set by $\widetilde{\sigma}$. This set will be systematically used from now on.

Let $\tilde{\sigma}$ be a set obtained by adding to $\sigma^{*} n$ arcs $\sigma_{i}^{\prime}$ which lie in the sectors $\Sigma_{i}$ and connect the origin $O=P_{*}$ - the center point of $\sigma$ - with the cycles 


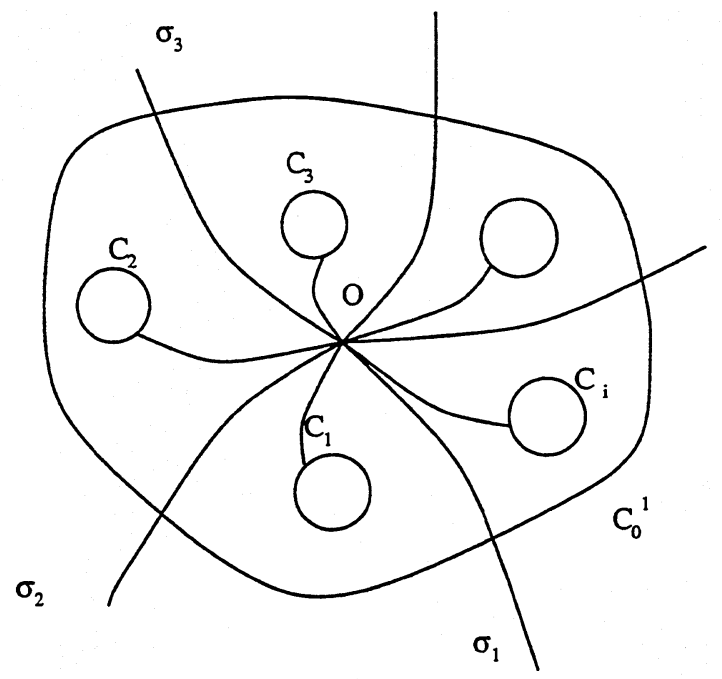

Figure 5: The set $\widetilde{\sigma}$

$C_{i}^{1}, i=1, \ldots, n$. It is assumed that $\sigma_{i}^{\prime}$ have only one point, namely $P_{*}$, of intersection with $\sigma^{*}$ and only one point of intersection with $C_{i}$ and $C_{i}^{1}$. This $2 n$-leg spider set $\widetilde{\sigma}$ is contractible and $\pi_{1}\left(D^{\prime}, \widetilde{\sigma}\right)=\pi_{1}\left(D^{\prime}, \sigma^{*}\right)=\pi_{1}$. Note that the set $\widetilde{\sigma}$ divides $\bar{D}^{\prime}$ into $n$ contractible connected components $\Omega_{i}$. If the type $h\left(\left.u\right|_{\left[s_{1}, s_{1}^{\prime}\right]}\right) \in \pi_{1}$ of a restriction of $u(s)$ on $\left[s_{1}, s_{1}^{\prime}\right]$ does not coincide with $g_{i}^{ \pm 1}$ or with 1 , there is one more point of intersection with $\widetilde{\sigma}$ : $u\left(s_{*}\right) \in \widetilde{\sigma}, s_{1}<s_{*}<s_{1}^{\prime}$. Indeed, if there is no intersection with $\widetilde{\sigma}$ then this subcurve lies in one $\Sigma_{i}$ and belongs to $g_{i}^{\alpha}$. If $|\alpha|>1$ there is a point of self-intersection which gives a closed loop with a type of class $g_{i}^{ \pm 1}$ (see the proof of Lemma 3.1) which lies in the $\Sigma_{i}$ and does not intersect $\tilde{\sigma}$. It is of nontrivial type when $C_{i}^{1}$ is inside the loop; but $C_{i}^{1}$ is connected with $C_{0}$ by $\tilde{\sigma}$, which must intersect the loop. So $|\alpha|=1$. Therefore on every essential interval $h\left(\left.u\right|_{\left[s_{l}, s_{l}^{\prime}\right]}\right)=g_{i_{l}}^{\delta_{l}}, \delta_{l}= \pm 1$. So we can write (4.7) with $\sigma=\tilde{\sigma}$ in the form

$$
h_{\sigma}(u)=g_{i_{1}}^{\delta_{1}} g_{i_{2}}^{\delta_{2}} \ldots g_{i_{n}}^{\delta_{n}}=\prod_{l=1}^{N_{1}} g_{i_{l}}^{\delta_{l}} \in \tilde{\pi}_{1}, g_{i_{l}}^{\delta_{l}}=h\left(\left.u\right|_{\left[s_{l}, s_{l}^{\prime}\right]}\right)=g_{i_{l}}^{ \pm 1}
$$

Theorem 4.3. Let $L>0$. Let $b \in \pi_{1}$. Let $\mathcal{L}<\ell^{*}\left(b, p_{1}, p_{2}\right)+\theta$. Then the set $X=\Xi_{b}^{\prime}\left([0, L], p_{1}, p_{2}\right) \cap\{\mathcal{E}(u) \leq \mathcal{L}\}$ is invariant under the dynamics of (1.1), (2.3) that is $u(t) \in \Xi_{b}^{\prime}\left([0, L], p_{1}, p_{2}\right) \cap\{\mathcal{E}(u) \leq \mathcal{L}\}$ for all $t>0$ if it 
is true for $t=0$. If this set $X$ is non-empty, it contains the attractor $\mathcal{A}$ which includes a stable equilibrium point $Z$ which is a solution of (1.2) and a global minimizer of $\mathcal{E}$ over $X$.

Proof. The invariance and existence of the attractor and of $Z$ directly follow from Theorem 3.1 and Theorem 2.2.

Remark 4.1. Obviously we can assume that $F(u)$ may be not positive inside the cycles $C_{i}^{1}$; in fact we may allow $F(u)$ to be equal to zero at isolated points inside $C_{0}$, but in this case we can't assert existence of periodic solutions of arbitrary type; solutions of homoclinic or heteroclinic type can arise instead, thanks to unboundedness of $L_{j}$. See for this case [4].

Remark 4.2. When a word $b$ contains at least two different generators, a curve from this class which has a point on $C_{i}^{1}$ must intersect $C_{i}$. Therefore one may slightly relax conditions of minimality and $\theta$-stability of the minimal cycle in Definitions 3.1 and 3.2. Namely, the conditions should be imposed only on curves which intersect $C_{i} ; \epsilon_{2}$ and $\theta$ may be put to zero for curves $\Gamma$ which do not intersect the cycle $C_{i}$. The statements of Theorems 3.1, 4.1, 4.2 and 4.3 remain true for such $b$ under the relaxed conditions.

\section{Equations on the infinite interval.}

From now on we take the set $\tilde{\sigma}$ given in the previous section as a base set $\sigma$.

Consider now solutions of (1.2) and (1.1) on the infinite interval $-\infty<$ $x<\infty$. We take a generalized element of the fundamental group of $D^{\prime}$ in the form of an infinite word

$$
b=\prod_{l=-\infty}^{\infty} g_{i_{l}}^{\kappa_{l}} \in \pi_{1}^{\infty}
$$

where $\kappa_{l}= \pm 1$ and $\kappa_{l+1}=\kappa_{l}$ if $i_{l+1}=i_{l}$; the last condition means that the word is irreducible.

A continuous function $u(x)$ belongs to $b \in \pi_{1}^{\infty}$ if there exists a sequence $-L_{N}^{-} \rightarrow-\infty, L_{N}^{+} \rightarrow \infty, N \rightarrow \infty$ such that $u\left(-L_{N}^{-}\right), u\left(L_{N}^{+}\right) \in \sigma$ and the restriction of $u(x)$ on the interval $\left[-L_{N}^{-}, L_{N}^{+}\right]$belongs to the class

$$
b_{N}=\prod_{l=-N}^{N} g_{i_{l}}^{\kappa_{l}} \in \pi_{1} .
$$


First we prove for every $b \in \pi_{1}^{\infty}$ existence of a solution $U$ of (1.2) from the class $b$.

Recall that for every $w \in \tilde{\pi}_{1}$ there exists an unique $b \in \pi_{1}$ such that $w \sim b$, that is $b$ is obtained from $w$ by cancellations of $g_{i} g_{i}^{-1}$ or $g_{i}^{-1} g_{i}$.

Proposition 5.1. Let $w \in \tilde{\pi}_{1}$. Let $w \sim b=b_{1} b_{2} \in \pi_{1}$. Then $w=w_{1} w_{2}$ where $w_{1} \sim b_{1}, w_{2} \sim b_{2}$.

Proof. Note that if a word $w \sim b$ where $b$ is irreducible, one obtains $b$ by deleting adjacent pairs of mutually inverse letters $g_{i}^{1}, g_{i}^{-1}$ from $w$ until there is none left. The word $b$ consists of the remaining letters of $w$. Inversely, inserting the pairs into their old positions we obtain from $b$ the word $w$. Let $c_{*}$ be the last letter of the subword $b_{1}$ of $b$. We take as the subword $w_{1}$ the subword of $w$ which starts with the first letter of $w$ and ends with $c_{*}$. Clearly it is obtained from $b_{1}$ by inserting in $b_{1}$ the canceling pairs to the left of $c_{*}$, that is $b_{1} \sim w_{1}$. The remaining letters of $w$ give $w_{2}$.

We denote by $\widehat{\sigma}$ a closed contractible subset of $\bar{D}^{\prime}$, which contains a $\delta$-neighborhood (Euclidean) in $\bar{D}^{\prime}$ of the set $\sigma$. Obviously, $\pi_{1}\left(D^{\prime}, \sigma\right)=$ $\pi_{1}\left(D^{\prime}, \widehat{\sigma}\right)=\pi_{1}$. Moreover, $\ell^{*}\left(b, p_{1}, p_{2}\right)$ is defined for $b \in \pi_{1}, p_{1}, p_{2} \in \widehat{\sigma}$.

Theorem 5.1. Let $b \in \pi_{1}^{\infty}$. Then there exists a steady-state solution $U(x),-\infty<x<\infty$, of the equation (1.2) from the class $b$. It satisfies (1.3); it lies in $\bar{D}^{\prime \prime}$ and $U(0) \in \sigma$. This solution takes values in $D^{\prime \prime}$ if it does not coincide with a periodic solution which coincides with one of minimal cycles $C_{i}$. If for $x_{1}<x_{2}$ we have $U\left(x_{1}\right)=p_{1}^{\prime} \in \sigma, U\left(x_{2}\right)=p_{2}^{\prime} \in \sigma$ then (4.6) holds.

If $F^{\prime \prime}(u)$ is continuous, the solution is stable in $\mathcal{L}_{2}(\mathbf{R})$ that is

$$
\int_{-\infty}^{\infty}\left[\partial_{x}^{2} V(x)-F^{\prime \prime}(U) V(x)\right] V(x) d x \leq 0
$$

for any smooth function $V(x)$ with a compact support.

Proof. Let $b_{j}^{-}=\prod_{l=-j}^{0} g_{i_{l}}^{\kappa_{l}}, b_{j}^{+}=\prod_{l=1}^{j} g_{i_{l}}^{\kappa_{l}}, b_{j}=b_{j}^{-} b_{j}^{+}$. By Theorem 4.2 there exist $L_{j}^{\prime}$ such that $U^{j} \in b_{j}$ are solutions of (1.2) with the boundary condition $U(0)=U\left(L_{j}^{\prime}\right)=P_{*} ; U^{j}$ are minimizers of $\mathcal{E}$ from the class $b_{j}=h\left(\left.U^{j}\right|_{\left[0, L_{j}^{\prime}\right]}\right)$. Let $w_{j}=h_{\sigma}\left(\left.U^{j}\right|_{\left[0, L_{j}^{\prime}\right]}\right) \sim b_{j}$. 
By Proposition $5.1 w_{j}=w_{j}^{-} w_{j}^{+}$with $w_{j}^{-} \sim b_{j}^{-}, w_{j}^{+} \sim b_{j}^{+}$. According to (4.9) we have a one-to-one correspondence between the letters $g_{i_{l}}^{\delta_{l}}$ of the word $w_{j}$ and essential intervals $\left[x_{l}, x_{l}^{\prime}\right]$ of $U^{j}$. On taking the $L_{j}^{\prime \prime}=x_{l}^{\prime}$ where $l$ is the label of the last letter in $w_{j}^{-}$we obtain

$$
h\left(\left.U^{j}\right|_{\left[0, L_{j}^{\prime \prime}\right]}\right)=b_{j}^{-}, h\left(\left.U^{j}\right|_{\left[L_{j}^{\prime \prime}, L_{j}^{\prime}\right]}\right)=b_{j}^{+} .
$$

Note that since (1.2) does not depend explicitly on $x, U(x+y)$ is also a solution on $\left[-y, L_{j}^{\prime}-y\right]$. We take $y=L_{j}^{\prime \prime}$ and put $L_{j}^{-}=L_{j}^{\prime \prime}, L_{j}^{+}=$ $L_{j}^{\prime}-L_{j}^{\prime \prime}$. Note that the Euclidean length of an arc of $U^{j}$ corresponding to every essential interval is bounded from below by a constant $l_{E}^{*}$. By Lemma 3.3 the length of an essential interval is bounded from below by $\left(l^{*}\right)^{2} /\left(2 R_{1}\right)=c$ where $R_{1}$ is the same as $R_{d}, d=1$, in Proposition 4.1. Therefore the lengths $L_{j}^{-}$and $L_{j}^{+}$of supports of both halves of $U^{j}$ are not less than $j c, c>0$ and $L_{j}^{ \pm} \rightarrow \infty$ as $j \rightarrow \infty$.

Now we choose appropriate subsequences of $j$ which yield the homotopy type $b$ for the limit function. Let us fix an integer $N$ and consider $j>N$. We take $b_{1}^{\prime}=b_{N}^{-}=\prod_{l=-N}^{0} g_{i_{l}}^{\kappa_{l}}$ and obtain the decomposition $b_{j}^{-}=b_{1 j}^{\prime \prime} b_{1}^{\prime}$. By Proposition $5.1 w_{j}^{-}=w_{1 j}^{\prime \prime} w_{1}^{\prime}$ with $w_{1 j}^{\prime \prime} \sim b_{1 j}^{\prime \prime}$ and $w_{1}^{\prime} \sim b_{1}^{\prime}$. From (4.9) we have $h\left(\left.U^{j}\right|_{\left[-L_{j, N}^{-}, 0\right]}\right)=b_{1}^{\prime}$, clearly $\operatorname{deg}\left(b_{1}^{\prime}\right)=N+1$. By Proposition 4.1 $\mathcal{J}\left(\left.U^{j}\right|_{\left[-L_{j, N}^{-}, 0\right]}\right) \leq R_{N+1}+\theta$ and by Lemma 3.3 the numbers $L_{j, N}^{-}$are bounded uniformly in $j$. We choose a subsequence convergent to $L_{N}^{-}$. Similarly writing $b_{j}^{+}=b_{2}^{\prime} b_{2 j}^{\prime \prime}$ with $h\left(\left.U^{j}\right|_{\left[0, L_{j, N}^{+}\right]}\right)=b_{2}^{\prime}$ we choose a subsequence $j$ for which $L_{j, N}^{+} \rightarrow L_{N}^{+}$. Using a diagonal process we obtain a subsequence of $j$ for which we have the convergence for every $N$.

We fix an arbitrary $L>0$. The functions $U^{j}$ are bounded uniformly in $j$ in $\mathcal{C}$ (their graphs are contained in the bounded set $\bar{D}_{0}$ ). Since the parameterization is defined by $\left|\partial_{x} u\right|=2 F(u)$, we have boundedness in $\mathcal{C}^{1}([-L, L])$; expressing $\partial_{x}^{2} u$ from the equation (1.2) we obtain uniform boundedness in $\mathcal{C}^{2}([-L, L])$. Therefore on every finite interval $[-L, L]$ we easily deduce weak compactness of $\left\{U^{j}\right\}$ in $H_{2}([-L, L])$. Using a diagonal process we choose a subsequence of solutions $U^{j}$ of $(1.2)$ which converges weakly in $H_{2}([-L, L])$ and strongly in $H_{1}([-L, L]), \mathcal{C}([-L, L])$ and in $\mathcal{C}^{1}([-L, L])$ on every finite interval $[-L, L]$ to a solution $U(x)$. Since graphs of $U^{j}$ are in $D^{\prime \prime}$, the graph of $U$ is in $\bar{D}^{\prime \prime}$.

Since $U^{j}\left(L_{j, N}^{ \pm}\right) \in \sigma$, from convergence in $\mathcal{C}([-L, L])$ it follows that 
$U\left(L_{N}^{ \pm}\right) \in \sigma$ and $U^{j}\left(L_{N}^{ \pm}\right) \in \widehat{\sigma}$ for large $j$. Since $\widehat{\sigma}$ is contractible

$$
h_{\widehat{\sigma}}\left(\left.U^{j}\right|_{\left[L_{N}^{-}, L_{N}^{+}\right]}\right) \sim h_{\sigma}\left(\left.U^{j}\right|_{\left[L_{j, N}^{-}, L_{j, N}^{+}\right]}\right) \sim \prod_{l=-N}^{N} g_{i_{l}}^{\kappa_{l}} .
$$

Therefore $h\left(\left.U^{j}\right|_{\left[L_{N}^{-}, L_{N}^{+}\right]}\right)=b_{N}$ does not depend on $j$ and we can pass to the limit and obtain

$$
h\left(\left.U\right|_{\left[-L_{N}^{-}, L_{N}^{+}\right]}\right)=\prod_{l=-N}^{N} g_{i_{l}}^{\kappa_{l}}
$$

that is $U \in b$.

Since (1.3) holds for $U^{j}$, by convergence in $\mathcal{C}^{1}$ we get it for $U$. The graph of $U$ is in $\bar{D}^{\prime \prime}$; the boundary of $D^{\prime \prime}$ consists of minimal cycles $C_{i}$. If the graph of $U$ intersects $C_{i}$ it should be tangent to it and by (1.3) the graph of $U$ should coincide with $C_{i}$ since they correspond to solutions of the same second order ODE with the same initial data.

One can pass to the limit in (4.3) for any fixed $V(x)$ with a compact support and obtain that the limit solution $U$ is stable in $\mathcal{L}_{2}(\mathbf{R})$.

Now we prove that (4.6) is true for $U$. Assuming that (4.6) does not hold for $U$ on $\left[x_{1}, x_{2}\right]$ when $U\left(x_{1}\right), U\left(x_{2}\right) \in \sigma$, that is

$$
\int_{x_{1}}^{x_{2}}\left[F(U(x))+\left|\partial_{x} U(x)\right|^{2} / 2\right] d x>\ell^{*}\left(b^{\prime}, p_{1}^{\prime}, p_{2}^{\prime}\right)+\epsilon_{1}, b^{\prime}=h\left(\left.U\right|_{\left[x_{1}, x_{2}\right]}\right)
$$

we will obtain a contradiction. Indeed, consider $U, U^{j}$ on the interval $\left[x_{1}, x_{2}\right]$. We have

$$
\left.\left|\mathcal{E}\left(\left.U\right|_{\left[x_{1}, x_{2}\right]}\right)-\mathcal{E}\left(\left.U^{j}\right|_{\left[x_{1}, x_{2}\right]}\right)\right| \leq\left. K_{2}|| U\right|_{\left[x_{1}, x_{2}\right]}-\left.\left.U^{j}\right|_{\left[x_{1}, x_{2}\right]}\right|_{H_{1}\left(\left[x_{1}, x_{2}\right]\right.}\right)
$$

where $K_{2}$ depends on bounds of $H_{1}\left(\left[x_{1}, x_{2}\right]\right)$-norms of $U, U^{j}$ on the fixed interval $\left[x_{1}, x_{2}\right]$ and is bounded. If $j$ is large enough, we obtain from (5.4)

$$
\int_{x_{1}}^{x_{2}}\left[F\left(U^{j}(x)\right)+\left|\partial_{x} U^{j}(x)\right|^{2} / 2\right] d x>\ell^{*}\left(b^{\prime}, p_{1}^{\prime}, p_{2}^{\prime}\right)+\epsilon_{1} / 2 .
$$

Since $U^{j}$ are global minimizers, we obtain from (4.6) (which is applicable to an arbitrary contractible $\sigma$, for example to $\widehat{\sigma}$ )

$$
\mathcal{E}\left(\left.U^{j}\right|_{\left[x_{1}, x_{2}\right]}\right)=\ell^{*}\left(b^{\prime}, p_{1}^{j}, p_{2}^{j}\right) .
$$


Comparing with (5.5) we obtain

$$
\ell^{*}\left(b^{\prime}, p_{1}^{j}, p_{2}^{j}\right)>\ell^{*}\left(b^{\prime}, p_{1}^{\prime}, p_{2}^{\prime}\right)+\epsilon_{1} / 2 .
$$

where $p_{1}^{j}=U^{j}\left(x_{1}\right), p_{2}^{j}=U^{j}\left(x_{2}\right)$ with $p_{1}^{j}, p_{2}^{j} \in \widehat{\sigma}$ since $\left.U^{j}\right|_{\left[x_{1}, x_{2}\right]}$ is in a $\delta$-neighborhood of $\left.U\right|_{\left[x_{1}, x_{2}\right]}$ in the $\mathcal{C}$-norm.

On the other hand, the dependence of the length of minimizers with fixed endpoints on their endpoints has the following general property. If $p_{1}^{\prime}, p_{2}^{\prime} \in \sigma,\left|p_{1}^{\prime}-p_{1}\right|<\delta,\left|p_{2}-p_{2}^{\prime}\right|<\delta$, connecting $p_{1}$ with $p_{1}^{\prime}$ and $p_{2}$ with $p_{2}^{\prime}$ by straight line segments (which are in $\widehat{\sigma}$ by definition of $\widehat{\sigma}$ ) we obtain

$$
\left|\ell^{*}\left(b^{\prime}, p_{1}, p_{2}\right)-\ell^{*}\left(b^{\prime}, p_{1}^{\prime}, p_{2}^{\prime}\right)\right| \leq K_{1}\left(\left|p_{1}-p_{1}^{\prime}\right|+\left|p_{2}-p_{2}^{\prime}\right|\right)
$$

where

$$
K_{1}=\sup _{u \in \widetilde{\sigma}} \sqrt{2 F(u)}
$$

We have $p_{1}^{\prime}=U\left(x_{1}\right) \in \sigma, p_{2}^{\prime}=U\left(x_{2}\right) \in \sigma$. Since $U^{j} \rightarrow U$ on the interval $\left[x_{1}, x_{2}\right]$, points $p_{1}^{j}, p_{2}^{j}$ are close to $p_{1}^{\prime}, p_{2}^{\prime}$ and we can apply (5.7). Since $p_{1}^{j} \rightarrow$ $p_{1}^{\prime}, p_{1}^{j} \rightarrow p_{1}^{\prime}$, for large $j$ we have $\left|\ell^{*}\left(b^{\prime}, p_{1}^{j}, p_{2}^{j}\right)-\ell^{*}\left(b^{\prime}, p_{1}^{\prime}, p_{2}^{\prime}\right)\right|<\epsilon_{1} / 2$. This contradicts (5.6). This contradiction shows that (5.4) cannot hold.

Lemma 5.1. Let (3.5) hold on an interval $\left[x_{1}, x_{2}\right] \subset[0, L]$. Then

$$
\sqrt{L_{0}} \leq \frac{\sqrt{2 \theta}+\sqrt{2 \theta+4 \mathcal{J}_{0}}}{2 \sqrt{2 \mu}}
$$

where $L_{0}=\left|x_{2}-x_{1}\right|, \mathcal{J}_{0}$ is the Jacobian length of the curve $u$ on $\left[x_{1}, x_{2}\right]$.

Proof. We have

$$
\begin{aligned}
\int_{x_{1}}^{x_{2}}\left[\sqrt{2 \mu}-\left|\partial_{x} u\right|\right] d x \leq & \int_{x_{1}}^{x_{2}}\left[\sqrt{2 F(u)}-\left|\partial_{x} u\right|\right] d x \\
& \leq \int_{x_{1}}^{x_{2}}|\sqrt{2 F(u)}-| \partial_{x} u|| d x \leq \sqrt{x_{2}-x_{1}} \sqrt{2 \theta} .
\end{aligned}
$$

This implies

$$
L_{0} \sqrt{2 \mu} \leq \sqrt{L_{0}} \sqrt{2 \theta}+\int_{x_{1}}^{x_{2}}\left|\partial_{x} u\right| d x \leq \sqrt{L_{0}} \sqrt{2 \theta}+\int_{x_{1}}^{x_{2}}\left|\partial_{x} u\right| \frac{\sqrt{2 F(u)}}{\sqrt{2 F(u)}} d x .
$$


Therefore

$$
L_{0} \sqrt{2 \mu} \leq \sqrt{L_{0}} \sqrt{2 \theta}+\frac{1}{\sqrt{2 \mu}} \mathcal{J}_{0}, \mathcal{J}_{0}=\int_{x_{1}}^{x_{2}}\left|\partial_{x} u\right| \sqrt{2 F(u)} d x .
$$

This expression is quadratic in $\sqrt{L_{0}}$ and after solving a quadratic equation we obtain the estimate (5.9).

Consider now the parabolic equation (1.1) in the unbounded domain $x \in \mathbf{R}$. We will show that together with a steady-state solution $Z$ with a given homotopy type and a fixed value of the Hamiltonian given by (1.3) there exists an open in appropriate topology invariant set of the parabolic equation consisting of functions which have the same homotopy type. It gives nonlinear stability for the spatial pattern of solutions.

Recall that the norm in the weighted Sobolev space $H_{k,-p}$ is defined by

$$
\|u\|_{H_{k,-p}}^{2}=\int_{-\infty}^{\infty}\left[\left(1+|x|^{2}\right)^{-p / 2} \sum_{j=0}^{k}\left|\partial_{x}^{j} u(x)\right|^{2} d x .\right.
$$

We extend the function $F(u)$ outside $D_{0}^{1}$ so that the first and second derivatives of $F(u)$ are bounded uniformly in the entire plane. From results of [7] we obtain:

Theorem 5.2. For any initial data $u(0) \in H_{1,-p}, p>1$, there exists a unique solution $u(t)=u(x, t)$ of (1.1) with $u(t) \in H_{1,-p}, t \geq 0$.

Therefore the dynamics $V \rightarrow \mathcal{S}_{t} V$ generated on $H_{1,-p}$ by (1.1) is welldefined. Obviously, bounded smooth functions, in particular smooth periodic in $x$ functions with an arbitrary period belong to $H_{1,-p}$ if $p>1$; periodic functions form invariant subspaces in this space. Since $U(x)$ is bounded and by (1.3) $\partial_{x} U(x)$ is bounded uniformly in $x$, solutions constructed in Theorem 5.1 belong to $H_{1,-p}$ if $p>1$.

Proposition 5.2. Let a curve $u(x)$ lie in $\bar{D}^{\prime}, p_{1}=u(0) \in \sigma, p_{2}=u(L) \in \sigma$. Let $\mathcal{E}(u) \leq \ell^{*}\left(h(u), p_{1}, p_{2}\right)+\zeta_{1}$ and (3.5) hold. Then there exists such $a$ $L_{*}>0$, which does not depend on $u, L$, that if $y^{\prime}, y \in[0, L]$ satisfy $y^{\prime}-y>L_{*}$ then $u\left(x_{*}\right) \in \sigma$ for some $y<x_{*}<y^{\prime}$.

Proof. Note that by (3.1) $\mathcal{J}(u) \leq \ell^{*}\left(h(u), p_{1}, p_{2}\right)+\zeta_{1}$. The set $\sigma=\tilde{\sigma}$ divides $\bar{D}^{\prime}$ into $n$ disjoint contractible subdomains $\Omega_{i}, i=1, \ldots, n$. The internal 
diameter of every disjoint component $\Omega_{i}$ in $\mathcal{J}$-metric is bounded from above by a number $2 R_{\Omega}$. Changing an arc of the curve inside a component $\Omega_{i}$ with the endpoints of the arc fixed does not change the type of the entire curve. Consider an arc which lies in a component $\Omega_{i}$. If the Jacobian length of the arc is greater than $2 R_{\Omega}+\zeta_{2}, \zeta_{2}>\zeta_{1}$ we connect the endpoints of the arc by an arc in $\Omega_{i}$ with the length $2 R_{\Omega}+\epsilon$. So we get a new curve with the same type and the Jacobian length of this curve is less than $\ell^{*}\left(h(u), p_{1}, p_{2}\right)+\zeta_{1}-\zeta_{2}+\epsilon$ which contradicts the definition of $\ell^{*}$ when $\epsilon$ is small. Therefore, if the Jacobian length of an arc of $u$ is greater than $2 R_{\Omega}+\zeta_{1}$ it intersects $\sigma$. By (5.9), if

$$
\sqrt{L_{*}}>\frac{\sqrt{2 \theta}+\sqrt{2 \theta+4\left(2 R_{\Omega}+\zeta_{1}\right)}}{2 \sqrt{2 \mu}}
$$

we have the Jacobian length $\mathcal{J}_{0}>2 R_{\Omega}+\zeta_{1}$; therefore we have an intersection with $\sigma$.

Theorem 5.3. Let $b \in \pi_{1}^{\infty}$, let $Z=U \in b$ be a solution of (1.2) which has the properties described in Theorem 5.1. Let $V(x)$ be a function which has the following properties : $\|V-Z\|_{\mathcal{C}(\mathbf{R})} \leq \epsilon_{1}$;

$$
\left.\int_{-\infty}^{\infty}|F(V(x))+| \partial_{x} V(x)\right|^{2} / 2-F(U(x))-\left|\partial_{x} U(x)\right|^{2} / 2 \mid d x \leq \epsilon_{2}
$$

Let $\epsilon_{1} \leq \delta, \epsilon_{2}+K_{1} \epsilon_{1}=\theta_{1}<\theta$ where $K_{1}$ is given by (5.8). Then there exists a unique solution $u(x, t)$ of (1.1) such that $u(x, 0)=V(x), u(x, t) \in$ $\bar{D}^{\prime} \forall x, t$ and for every fixed $t=t_{0} \geq 0 u\left(t_{0}\right) \in b$. This solution satisfies the approximate Hamiltonian conservation law

$$
\int_{-\infty}^{\infty}\left(\sqrt{2 F(u(x, t))}-\left|\partial_{x} u(x, t)\right|\right)^{2} d x \leq 2 \theta \quad \forall t \geq 0
$$

and (4.4), (4.5) with $\zeta=\theta$.

Proof. Let $L_{j}^{-}, L_{j}^{+}$be the same as in the proof of Theorem 5.1 with $U=Z$. We have $Z\left(-L_{j}^{-}\right)=q_{1}, Z\left(L_{j}^{+}\right)=q_{2}, Z(0)=q_{3}$ with $q_{1}, q_{2}, q_{3} \in \sigma$. The function $Z_{j}(x)=\left.Z\right|_{\left[-L_{j}^{-}, L_{j}^{+}\right]}$on the interval $\left[-L_{j}^{-}, L_{j}^{+}\right]$has the homotopy type $h\left(Z_{j}\right)=b_{j}=\prod_{l=-j}^{j} g_{i_{l}}^{\kappa_{l}}$. We have

$$
h\left(\left.Z\right|_{\left[-L_{j}^{-}, 0\right]}\right)=\prod_{l=-j}^{0} g_{i_{l}}^{\kappa_{l}}=b_{j}^{-}, \quad h\left(\left.Z\right|_{\left[0,-L_{j}^{+}\right]}\right)=\prod_{l=1}^{j} g_{i_{l}}^{\kappa_{l}}=b_{j}^{+}, \quad b_{j}=b_{j}^{-} b_{j}^{+} .
$$


We restrict initial data $V(x)$ to the interval $\left[-L_{j}^{-}, L_{j}^{+}\right]$with $V\left(-L_{j}^{-}\right) \in$ $\widehat{\sigma}, V\left(L_{j}^{+}\right) \in \widehat{\sigma}$ since $\epsilon_{2} \leq \delta$.

Consider (1.1) with the Dirichlet boundary condition

$$
u\left(-L_{j}^{-}, t\right)=V\left(-L_{j}^{-}\right)=p_{1}^{j}, u\left(L_{j}^{+}, t\right)=V\left(L_{j}^{+}\right)=p_{2}^{j} \forall t \geq 0
$$

and with the initial condition $u(x, 0)=V(x), x \in\left[-L_{j}^{-}, L_{j}^{+}\right]$.

By Theorem 4.3 we have $u_{j}(t) \in X=\Xi_{b}^{\prime}\left(\left[-L_{j}^{-}, L_{j}^{+}\right], p_{1}^{j}, p_{2}^{j}\right) \forall t \geq 0$, therefore $u_{j}(x, t) \in D^{\prime}$ are bounded uniformly in $x, t, j$, that is we have an uniform estimate in $\mathcal{C}$. For any fixed $L, T$ with $L_{j}^{ \pm} \geq L+1$ we consider solutions $u_{j}$ on the rectangle $Q=\{-L \leq x \leq L\} \times\{0 \leq t \leq T\}$. The main part of the equation (1.1) is linear and the nonlinear term $F^{\prime}(u)$ is bounded for bounded $u$ and can be considered as a given function. Using uniform boundedness of initial and boundary data, Lipschitz dependence of $F^{\prime}(u)$ on $u$ and standard properties of linear parabolic equations (see [19], [17]) we obtain uniform in $j$ boundedness of the first order time derivative and the second order space derivatives of solutions in $\mathcal{C}^{\gamma}(Q), \gamma>0$. We can choose a subsequence of solutions of (1.1) which converges in $\mathcal{C}(Q)$ and the time derivative and second order space derivatives converge in $\mathcal{L}_{2}(Q)$ as $j \rightarrow \infty$; the limit function $u(x, t)$ is again a solution of $(1.1), u(x, 0)=V(x)$. Since $u^{j}(x, t) \in \bar{D}^{\prime} \forall x, t, j$ it is true for $u(x, t)$. So we have proved the existence of a solution. Its uniqueness follows from Theorem 5.2. Since every subsequence converges to the same limit $u(x, t)$, the sequence $u^{j}(x, t) \rightarrow u(x, t)$ in $\mathcal{C}(Q)$ together with first time and space derivatives.

The inequality (5.11) follows in the limit from (3.5). We obtain for $u^{j}$ (4.4), (4.5) from Proposition 4.2 with $\zeta=\theta$. Passing to the limit like in the proof of (4.6) in Theorem 5.1 we obtain (4.4), (4.5) for $u$.

Now we show that the solution $u(t) \in b$ for any fixed $t=T$. We use the same approach as in Theorem 5.1. We have

$$
h_{\sigma}\left(\left.u^{j}(t)\right|_{\left[L_{j}^{-}, L_{j}^{+}\right]}\right)=\prod_{l=-J_{-}}^{J_{+}} g_{i_{l}}^{\delta_{l}}=w^{j} \sim b_{j} .
$$

Since $b_{j}=b_{j}^{-} b_{j}^{+}$we have by Proposition $5.1 w_{j}=w_{j}^{-} w_{j}^{+}$with $w_{j}^{-} \sim b_{j}^{-}$. The last essential interval corresponding to the last letter of $w_{j}^{-}$has the right endpoint $L_{j}^{0}, u^{j}\left(L_{j}^{0}, T\right) \in \sigma$.

We show that $L_{j}^{0}$ are bounded uniformly in $j$. Consider the rectangle $Q_{j}^{-}=\left\{-L_{j}^{-} \leq x \leq 0\right\} \times\{0 \leq t \leq T\}$. The function $u(x, t)$ restricted to its boundary determines a cycle $\Gamma$ in the $u$-plane which lies in $D^{\prime}$. The 
cycle consists of four arcs $\Gamma_{1}, \Gamma_{2}, \Gamma_{3}, \Gamma_{4}$ corresponding to the lower, the right, the upper and the left sides of the rectangle respectively. According to the boundary condition, $u\left(\Gamma_{4}\right)=Z\left(-L_{j}^{-}\right) \in \sigma$. The cycle $\Gamma$ has the trivial homotopy type. Indeed, by decreasing $T$ we obtain a homotopy which at $T=0$ yields a trivial cycle obtained by passing the same curve first from left to right (from $-L_{j}^{-}$to 0 ) and then backwards; this cycle can be contracted by moving the right endpoint of the curve along the curve to the left endpoint. Therefore the homotopy classes corresponding to the curves $\Gamma_{1}$ and $\Gamma_{2} \cup \Gamma_{3}=$ $\Gamma_{5}$ are inverse. Changing the orientation on $\Gamma_{5}$ to the opposite (positive direction on the upper side now corresponds to increasing $x$ ), we obtain that the classes coincide.

By Proposition 5.2 we have $x_{0} \in\left[-L_{*}, 0\right], u\left(x_{0}, T\right)=p_{0} \in \sigma$, we take the maximum of all such $x_{0} ;$ let $h\left(\left.u_{j}\right|_{\left[-L_{j},-L_{*}\right]}\right)=b_{0 j}$. The irreducible word $b_{0 j}$ is a subword of the irreducible word $b_{j}, b_{0 j} b_{0 j}^{\prime}=b_{j}$. We have two possibilities, $b_{0 j} \subset b_{j}^{-}$and $b_{0 j}^{\prime} \subset b_{j}^{+}$. We consider the first case, the second is similar (we need to consider $x \geq 0$ then).

The curve $\Gamma_{5}$ consists of three parts: $u_{1}$ given by $u^{j}(x, T),-L_{j}^{-} \leq x \leq x_{0}$; $u_{2}$ given by $u^{j}(x, T), x_{0} \leq x \leq 0$ and $u_{3}$ given by $u(0, t), T \geq t \geq 0$. We denote the union of $u_{2}, u_{3}$ by $u_{4}$. So we have $b_{j}^{-}=b_{0 j} b_{j}^{\prime \prime}$ with $b_{j}^{\prime \prime}=h\left(u_{4}\right)$. Recall that $h\left(\left.Z\right|_{\left[-L_{j}^{-}, 0\right]}\right)=h\left(\Gamma_{5}\right)=b_{j}^{-}$. We have $h_{\sigma}\left(\Gamma_{5}\right)=w_{j}^{-} \sim b_{j}^{-}$and by Proposition $5.1 w_{j}^{-}=w_{0 j}^{-} w^{\prime \prime}, w^{\prime \prime} \sim b_{j}^{\prime \prime}$.

Now we prove that the degree of $b_{j}^{\prime \prime}$ is bounded uniformly in $j$. Indeed, the Euclidean length of the curve $\Gamma_{2}$ is bounded by $\int_{0}^{T}\left|\partial_{t} u_{j}(0, t)\right| d t$, and this integral is bounded uniformly since $\partial_{t} u_{j}(x, t)$ are bounded in $Q$. Since on $x_{0}<x<0<x_{1} u_{j}(x) \notin \sigma$, with $u_{j}\left(x_{0}\right) \in \sigma, u_{j}\left(x_{1}\right) \in \sigma$ it has type $g_{i}^{ \pm 1}$ or 1 and by Proposition 4.1 it has a Jacobian length bounded by $R_{1}$. Therefore the Euclidean and Jacobian lengths of $\Gamma_{5}$ are bounded, which implies that $\operatorname{deg}\left(h\left(u_{4}\right)\right) \leq d_{1}(T)$. Therefore $\operatorname{deg} b_{j}^{\prime \prime} \leq d_{1}(T)$ and $\operatorname{deg}\left(h\left(\left.u^{j}\right|_{\left[-L_{*}^{-}, L_{j}^{0}\right]}\right)\right) \leq$ $d_{1}(T)$. Applying Proposition 4.1 we obtain that $\left.\mathcal{J}\left(\left.u^{j}\right|_{\left[-L_{*}^{-}, L_{j}^{0}\right]}\right)\right) \leq K^{\prime}$ and by Lemma $5.1\left|L_{j}^{0}\right| \leq K_{1}^{\prime}$ are bounded uniformly in $j$.

Now we define $L_{N}^{-}$. We have

$$
h_{\sigma}\left(\left.u^{j}(t)\right|_{\left[L_{j}^{-}, L_{j}^{0}\right]}\right)=\prod_{l=-J_{-}}^{J_{0}} g_{i_{l}}^{\delta_{l}}=w_{j}^{-} \sim b_{j}^{-}=\prod_{l=-j}^{0} g_{i_{l}}^{\kappa_{l}} .
$$

Since $b_{j}^{-}=b_{N *}^{-} b_{N}^{-}$, using Proposition 5.1 we split $w_{j, N}=w_{j, N *}^{-} w_{j, N}^{-}$. Like in the proof of Theorem 5.1 for a fixed $t=T$ and $1 \leq N \leq j$ we can find points $L_{j, N}^{-}$, which now depend on $t$, such that $u^{j}\left(L_{j, N}^{-}, t\right) \in \sigma, L_{j, N}^{-}<L_{j}^{0}$, 
and

$$
h_{\sigma}\left(\left.u^{j}(t)\right|_{\left[L_{j, N}^{-}, L_{j}^{0}\right]}\right)=\prod_{-J_{N}}^{J_{0}} g_{i_{l}}^{\delta_{l}}=w_{j, N}^{-}(t) \sim b_{N}^{-} .
$$

Therefore deg $\left(h\left(\left.u^{j}(t)\right|_{\left[L_{j, N}^{-}, L_{j, N}^{0}\right]}\right)\right) \leq N+1$. By Proposition 4.1 the Jacobian length of corresponding curves is bounded by a constant which depends on $N$ and not on $j$. By Lemma 5.1 corresponding $x$-lengths are bounded too.

So $L_{j, N}^{-}$(and similarly $L_{j, N}^{+}$) are bounded uniformly in $j$ and we can choose subsequences converging to $L_{N}^{ \pm}$. We obtain in the limit $h\left(\left.u^{j}\right|_{\left[-L_{N}^{-}, L_{N}^{0}\right]}\right)=b_{N}^{-}$and similarly $h\left(\left.u^{j}\right|_{\left[L_{N}^{0}, L_{N}^{+}\right]}\right)=b_{N}^{+}$which implies that $u \in b$.

The steady-state solutions of (1.1) are obtained as local minimizers of energy with a prescribed value $\mu$ of the Hamiltonian. The solutions obtained in Theorem 5.1 are parameterized by two parameters: $\mu$ (it affects solutions through the potential $F(u)=F_{0}(u)+\mu$ and corresponding Jacobian metric) and by the homotopy type $b \in \pi_{1}^{\infty}$ which describes their spatial structure. From Theorem 5.3 follows that the constructed steady state $U(x)$ with a given $\mu, b$ is stable with respect to $H_{1,0}(\mathbf{R}) \cap H_{0,1}$-perturbations. Indeed, if $V-U$ has a small norm in this space then (5.10) holds as well as an estimate in $\mathcal{C}$.

Considering $\mu$ fixed, we study the spatial distribution of the homotopy elements of the set of solutions in the next section.

\section{Complexity of steady-state solutions.}

We want to describe how complexity of steady-state solutions we constructed in Theorem 5.1 is related to the length of an interval on which we consider the solution. First we give estimates of complexity in the general case. After that we consider in more detail the situation of Example 3.1, that is a locally radial potential.

For every element $b \in \pi_{1}^{\infty}$ we have by Theorem 5.1 a solution $Z \in$ $b, Z(0) \in \sigma$. Moreover, $Z_{[0, \infty)} \in b^{+}, Z_{(-\infty, 0)} \in b^{-}$with $b^{-}=\prod_{l=-\infty}^{0} g_{i_{l}}^{\kappa_{l}}$, $b^{+}=\prod_{l=1}^{\infty} g_{i_{l}}^{\kappa_{l}}$. We have constructed in Theorem 5.1 such $L_{N}^{-}, L_{N}^{+}$that

$$
Z_{\left[-L_{N}^{-}, 0\right]} \in b_{N}^{-}, b_{N}^{-}=\prod_{l=-N}^{0} g_{i_{l}}^{\kappa_{l}}, Z_{\left[0, L_{N}^{+}\right]} \in b_{N}^{+}, b_{N}^{+}=\prod_{l=1}^{N} g_{i_{l}}^{\kappa_{l}} .
$$

Therefore

$$
h\left(Z_{\left[L_{j-1}^{+}, L_{j}^{+}\right]}\right)=g_{i_{j}}^{\kappa_{j}}, h\left(Z_{\left[-L_{j}^{-},-L_{j-1}^{-}\right]}\right)=g_{i_{j}}^{\kappa_{j}} .
$$


We denote the set of all constructed $Z_{b}$ by $\mathcal{N}\left(\mathcal{N}\right.$ contains exactly one $Z_{b}$ for every $b$ ).

For every $Z_{b} \in \mathcal{N}$ and for any $i$ the lengths $\left|L_{j-1}^{ \pm}-L_{j}^{ \pm}\right|$of the intervals corresponding to $g_{i}^{ \pm 1}$ are uniformly bounded from below by a constant $L_{*}>0$ (since the type is not trivial) and from above by $L^{*}$ according to Proposition 4.1 and Lemma 3.3.

For every $L$ we denote by $L^{ \pm}(L)$ the maximum of $L_{j}^{ \pm}<L$. We denote $b^{\prime}(L)=h\left(Z_{\left[-L^{-}(L), L^{+}(L)\right]}\right) \in \pi_{1}$. We define the number $N(L)=N_{\mathcal{N}}(L)$ of different homotopy types of $z_{b} \in \mathcal{N}$ on $[-L, L]$ as the number of different $b^{\prime}(L)$.

Since the number of letters of the word $b^{\prime}(L)$ is between $2 L / L_{*}$ and $2\left(L / L^{*}-1\right)$ we have $N_{\mathcal{N}}(L) \leq 2 n(2 n-1)^{2 L / L_{*}-1}$ and $N_{\mathcal{N}}(L) \geq 2 n(2 n-$ $1)^{2 L / L^{*}-3}$.

We introduce a lower complexity $h_{*}(\mathcal{N})$ and an upper complexity $h_{*}(\mathcal{N})$

$$
h_{*}(\mathcal{N})=\lim \inf _{L \rightarrow \infty} \ln N_{\mathcal{N}}(L) /(2 L), h^{*}=\lim \sup _{L \rightarrow \infty} \ln N_{\mathcal{N}}(L) /(2 L) .
$$

So we obtain the following statement.

\section{Proposition 6.1.}

$$
h_{*}(\mathcal{N}) \geq \frac{\ln (2 n-1)}{L^{*}}, h^{*}(\mathcal{N}) \leq \frac{\ln (2 n-1)}{L_{*}} .
$$

where $n$ is the number of generators of the fundamental group $\pi_{1}\left(D^{\prime}, P_{*}\right)$.

Now we consider the situation of Example 3.1 (see also Fig. 6) when it is possible to give more precise information on $h_{*}(\mathcal{N})$. In this case the steady state solutions can be explicitly described up to a small error. Recall that we assume that $F$ is constant far from two points $P_{i}, i=1,2$; namely

$$
F(u)=\mu \text { for } u \notin D_{i}^{3}, D_{i}^{3}=\left\{\left|u-P_{i}\right|<\eta_{0}+d\right\} .
$$

Here $d>0$ is a small number and $F$ is radial inside $D_{i}^{3}$, that is (3.6) holds with $r_{3}=\eta_{0}+d$. It is assumed that (3.7) holds with $\epsilon=d$, that is the circles $C_{i}=\left\{\left|P_{i}-u\right|=\eta_{0}\right\}$ are minimal cycles.

We also assume that the variation

$$
d_{F}=\sup _{\eta_{0} \leq \eta \leq \eta_{0}+d}\left|\sqrt{\eta^{2} f(\eta)}-\sqrt{\eta_{0}^{2} f\left(\eta_{0}\right)}\right|
$$

is small. We denote by $\mu_{1}=\sqrt{\eta_{0}^{2} f\left(\eta_{0}\right)}$ the minimal value of $\sqrt{\eta^{2} f(\eta)}$ and assume that $d_{F} \leq \mu_{1} / 2$; we assume $d_{F} \leq c_{3} d$ with a fixed $c_{3}$. 


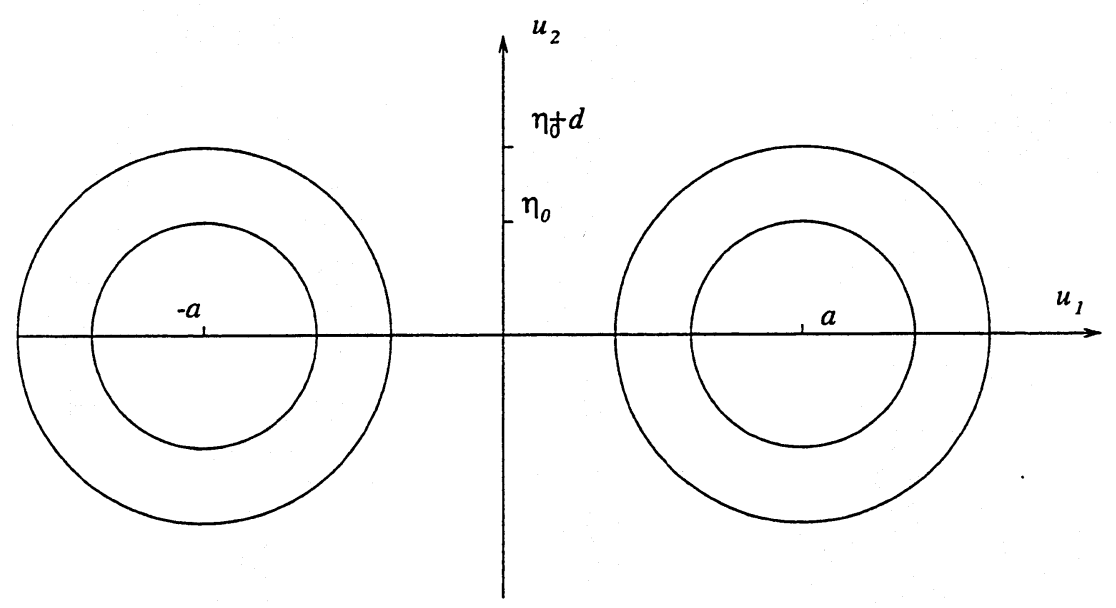

Figure 6.

We fix $\nu_{1}, \nu_{2}, 0<\nu_{1} \leq \eta_{0} / a \leq \nu_{2}<1$. Clearly, steady-state solutions $u(x)$ are linear when $u(x) \notin D_{i}^{3}$ and their graphs are straight line segments. At the same time, when $u(x) \in D_{i}^{3}$ by Theorem 4.1 the solutions lie in the rings $A_{i}=\left\{\eta_{0} \leq\left|u-P_{i}\right| \leq \eta_{0}+d\right\}$.

The set $\sigma^{*}$ is now the vertical segment $u_{1}=0,\left|u_{2}\right| \leq \eta+d$.

An external minimal cycle $C_{0}$ is inside the set formed by two horizontal straight lines $\left|u_{2}\right|=\eta_{0}+d$ and two semicircles $\left|u-P_{i}\right|=\eta_{0}+d,\left|u_{1}\right| \geq a$ (recall that $P_{i}=\left((-1)^{i} a, 0\right)$ ). The set $\tilde{\sigma}$ is obtained by adding to $\sigma^{*}$ the horizontal segment $\sigma^{\prime}=\left\{u_{2}=0,\left|u_{1}\right| \leq a-\eta_{0}\right\}$.

Since the function $F$ in the neighborhood of $P_{i}$ depends only on $\eta=$ $\left|P_{i}-u\right|$, the minimizers $u(x)=Z_{b}(x)$ in addition to minimality and (1.3) have special properties. We restrict ourselves to the case when $b$ includes powers of different $g_{i}$, that is $Z_{b}$ does not coincide with one of two simplest periodic solutions $b=g_{1}^{\infty}$ and $b=g_{2}^{\infty}$; the graphs of these simplest solutions coincide with minimal cycles $C_{1}$ and $C_{2}$. For nontrivial $b$ graphs of $u(x)=Z_{b}(x)$ always include straight-line segments (see Fig. 7).

Consider $u(x)$ on the interval $x_{1} \leq x \leq x_{2}$ when it is near $P_{i}=P_{1}$ and does not get into the second circle $D_{2}^{3}$. On this interval $u(x)$ can be considered as a solution of equation (1.2) with a globally radial potential.

From (3.13), (3.14) we obtain equations in polar coordinates

$$
\eta^{\prime \prime}-\eta\left(\phi^{\prime}\right)^{2}=f^{\prime}(\eta), \eta \phi^{\prime \prime}+2 \eta^{\prime} \phi^{\prime}=0
$$


The second equation implies Kepler's law

$$
\eta^{2} \phi^{\prime}=M_{1}=\text { const. }
$$

Therefore $\phi^{\prime}$ does not change sign and $\phi(x)$ is a monotone function.

Equation (1.3) in polar coordinates takes the form

$$
\left[\left(\eta^{\prime}\right)^{2}+\eta^{2}\left(\phi^{\prime}\right)^{2}\right]=2 f(\eta) .
$$

Denoting $\eta_{*}$ the minimal value of the solution $\eta(x)$ at $x=x_{0}, \eta_{*} \geq \eta_{0}$, we obtain from (6.2) at $x=x_{0}, \eta^{\prime}=0$ so $\left|\eta^{2} \phi^{\prime}\left(x_{0}\right)\right|=\sqrt{2 f(\eta) \eta^{2}}$. Putting $x=x_{0}$ in (6.1) we obtain $\eta^{2} \phi^{\prime}(x)=M_{1}= \pm \sqrt{2 \eta_{*}^{2} f\left(\eta_{*}\right)}$. $P_{i}$ :

We can rewrite $\mathcal{J}\left(\left.u\right|_{\left[x_{1}, x_{2}\right]}\right)$ using (1.3) in polar coordinates centered at

$$
\mathcal{J}\left(\left.u\right|_{\left[x_{1}, x_{2}\right]}\right)=\int_{x_{1}}^{x_{2}} 2 F(u(x)) d x=\int_{\phi_{1}}^{\phi_{2}} 2 \frac{f(\eta)}{\phi^{\prime}} d \phi
$$

with $\phi_{2} \geq \phi_{1}$. Expressing $\phi^{\prime}$ from (6.1) we obtain

$$
\mathcal{J}\left(\left.u\right|_{\left[x_{1}, x_{2}\right]}\right)=\int_{\phi_{1}}^{\phi_{2}} \frac{2 \eta^{2} f(\eta)}{\sqrt{2 \eta_{*}^{2} f\left(\eta_{*}\right)}} d \phi
$$

Similarly, we obtain an expression for the length of the $x$-interval

$$
L^{0}=x_{2}-x_{1}=\int_{x_{1}}^{x_{2}} d x=\int_{\phi_{1}}^{\phi_{2}} \frac{1}{\phi^{\prime}} d \phi .
$$

Therefore

$$
L^{0}=\int_{\phi_{1}}^{\phi_{2}} \frac{\eta^{2}}{\sqrt{2 \eta_{*}^{2} f\left(\eta_{*}\right)}} d \phi .
$$

Consider now $u(x)=Z_{b}(x)$ on an interval $\left[x_{-}, x_{+}\right]=\left[L_{j}^{+}, L_{l}^{+}\right]$corresponding to $h\left(\left.u\right|_{\left[L_{j}^{+}, L_{l}^{+}\right]}\right)=b^{\prime} \in \pi_{1}$. When $u(x)$ is outside $A_{i}$, the straight line $\sigma^{*}$ has only one transversal intersection with a straight-line segment of $u(x)$ during a transition from one ring $A_{i}$ into another. When $u(x)$ is inside $A_{i}, \phi$ is monotone and $u(x)$ intersects $\sigma^{\prime}|\alpha|-1$ times if the class is $g_{i}^{\alpha}$ between two transitions. Therefore $h_{\sigma}\left(\left.u\right|_{\left[L_{j}^{+}, L_{l}^{+}\right]}\right) \in \widetilde{\pi}_{1}$ is irreducible and coincides with $h\left(\left.u\right|_{\left[L_{j}^{+}, L_{l}^{+}\right]}\right) \in \pi_{1}$.

The points of possible intersection of a curve $Z$ with $\sigma=\tilde{\sigma}$ are located near five points: $p_{1}=\left(\eta_{0}-a, 0\right), p_{2}=\left(a-\eta_{0}, 0\right), p_{3}=(0,0)$, $p_{4}=\left(0, \eta_{0}\right), p_{5}=\left(0,-\eta_{0}\right)$. More precisely, the distance from these points 


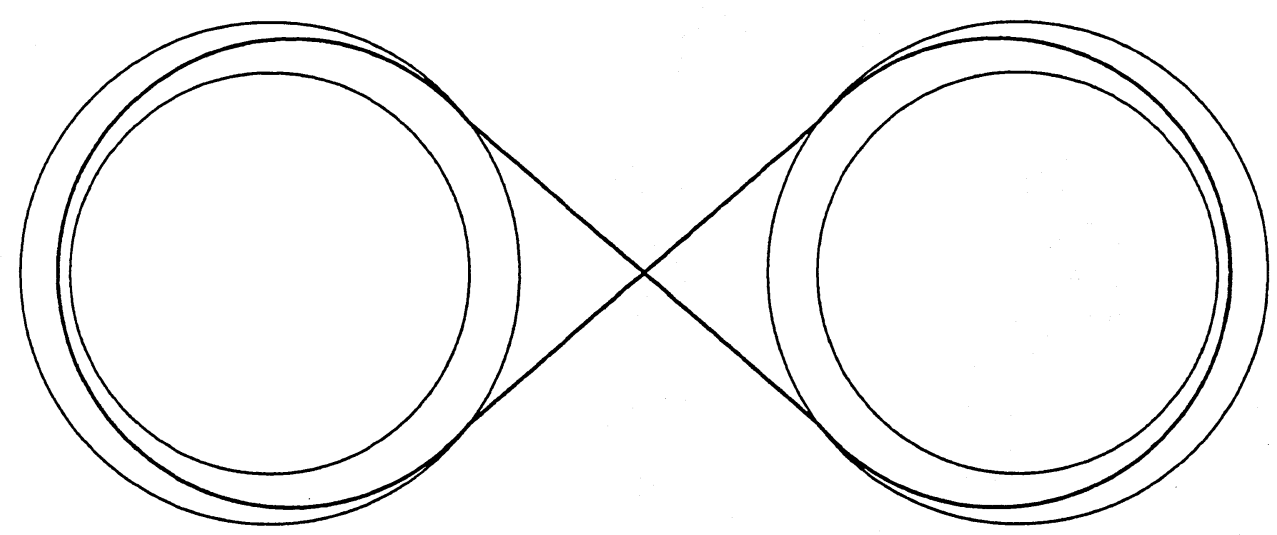

Figure 7: Curve $u(x)$

to the intersections is not greater than $c_{0} d, c_{0}=c_{0}\left(\nu_{1}, \nu_{2}\right)$. The restriction $\left(\left.u\right|_{\left[L_{j}^{ \pm}, L_{l}^{ \pm}\right]}\right)$has endpoints in $\tilde{\sigma}$ in a cod-neighborhood of one of these five points.

Therefore the angles $\phi_{1}, \phi_{2}$ which correspond to the intersection with $\tilde{\sigma}$ for fixed $\eta_{0}, a, b$ and depend on $f$ vary in an interval with a width bounded by $c_{1} d$ (angles corresponding to points of intersection with $\sigma^{\prime}$ do not vary at all). So the variation of the length $L^{0}$ from the same class for different $f$ given by (6.3) is bounded by $K_{3} c_{1} d+\left(\phi_{2}-\phi_{1}\right) K_{4}\left(d_{F}+d\right)$ where $K_{3}=$ $2 a^{2} / \mu_{1}, K_{4}=a^{2} / \mu_{1}^{2}+2 a / \mu_{1}$.

Fix $b$ and consider together with $u(x)=Z_{b}$ a curve $v=v_{b}$ which has the same type $b$ and has the same geometric properties as $u$ (monotonicity of $\phi$ in every $A_{i}$ ) but in the case $d=0$. That means that $v(s)$ is composed of arcs of minimal circles $C_{i}$ and of straight lines tangent to both of them. In the integral (6.3) for $v$ we take $f(\eta)=\mu$ (recall that for $Z_{b} f(\eta)=\mu$ when $\left.\eta \geq \eta_{0}+d\right)$. The parameterization of $v$ is obtained as a limit of (1.3) by putting $\left|\partial_{x} v\right|=\sqrt{2 \mu}$.

According to (6.3) the $x$ - lengths of $v$ with endpoints $Q_{ \pm}^{\prime} \in \tilde{\sigma}$ are the limit of the $x$-lengths of pieces $u=Z_{b}$ with endpoints $Q_{ \pm}$in $c_{0} d$ neighborhoods of $Q_{ \pm}^{\prime} \in \widetilde{\sigma}$ as $d \rightarrow 0, d_{F} \rightarrow 0$. The curve $v$ has the same type $b^{\prime} \in \pi_{1}\left(D^{\prime}, \widehat{\sigma}\right)$ as the arc of $v$ between $Q_{-}$and $Q_{+}$(we assume $c_{0} d \leq \epsilon$ and $\epsilon$ is so small that $\widehat{\sigma}$ does not intersect $\left.D_{i}^{1}, i=0, . ., n\right)$. The $x$-length $L(v)$ of $v$ is related with the Euclidean length $l_{E}(v)$ of this curve by the formula $L(v)=l_{E}(v) / \sqrt{2 \mu}$.

For any interval $\left[x_{-}, x_{+}\right]$with $Q_{ \pm}=u\left(x_{ \pm}\right) \in \tilde{\sigma}$ and with homotopy type $b^{\prime}$ on this interval $\left[x_{-}, x_{+}\right]$( $Q_{-}$is near one of the five points $p_{j}$ and $Q_{+}$is 


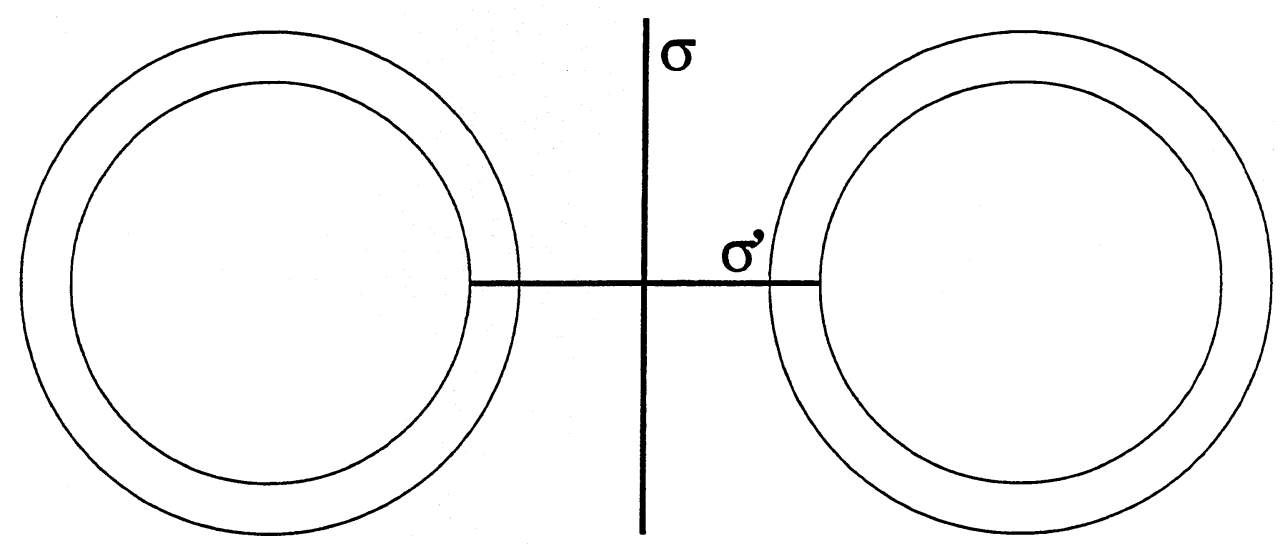

Figure 8: The set $\tilde{\sigma}$

near another $p_{l}$ ) we take $v_{b}$ from the same class $b ; v^{\prime}$ is an arc of $v$ from the class $b^{\prime}$ which starts near $p_{j}$ and ends near $p_{l}$. Applying the estimate of variation of $x$-lengths we obtain:

$$
\left|L^{0}\left(\left.u\right|_{\left[x_{-}, x_{+}\right]}\right)-l_{E}\left(v^{\prime}\right) / \sqrt{2 \mu}\right| \leq \operatorname{deg}\left(b^{\prime}\right) c_{1} d+K_{4} d_{F} \Delta \phi
$$

where $\Delta_{\phi}$ is the total variation of the angles $\phi_{i}$ in all $D_{i}$ along $u(x)$ on $\left[-x_{-}, x_{+}\right]$. We have $\Delta_{\phi} \leq 2 \pi \operatorname{deg} b^{\prime}$ where $\operatorname{deg} b^{\prime}$ is the degree of the word $b^{\prime}$. Since $L_{*}>0$ we have the estimate $\operatorname{deg} b^{\prime} \leq\left|x_{+}-x_{-}\right| / L_{*}$. Therefore we obtain the estimate which is uniform in $b^{\prime}$ :

$$
\left|L^{0}\left(\left.u\right|_{\left[x_{-}, x_{+}\right]}\right)-L^{0}(v)\right| \leq K_{5}\left(d_{F}+d\right)\left|x_{+}-x_{-}\right| / L_{*} .
$$

This implies

$$
\left|L^{0}(v) / L^{0}\left(\left.u\right|_{\left[x_{-}, x_{+}\right]}\right)-1\right| \leq K_{5}\left(d_{F}+d\right) / L_{*} \leq K_{6} d,
$$

when $d$ is small. Denoting the set of all $v_{b}$ by $\mathcal{N}^{\prime}$ we find the lower and the upper complexities $h_{*}\left(\mathcal{N}^{\prime}\right), h^{*}\left(\mathcal{N}^{\prime}\right)$. Since there is one-to-one correspondence between restrictions of $Z_{b}$ and related $v_{b}$ using (6.4) we conclude that

$$
\ln N_{\mathcal{N}}\left(\left(1+K_{6} d\right) L\right) \geq \ln N_{\mathcal{N}^{\prime}}(L), \quad \ln N_{\mathcal{N}}\left(\left(1-K_{6} d\right) L\right) \leq \ln N_{\mathcal{N}^{\prime}}(L)
$$

for large $L$ and small $d$. Therefore for small $d$

$$
\left|h_{*}\left(\mathcal{N}^{\prime}\right) / h_{*}(\mathcal{N})-1\right| \leq 3 K_{6} d ;\left|h^{*}\left(\mathcal{N}^{\prime}\right) / h^{*}(\mathcal{N})-1\right| \leq 3 K_{6} d .
$$


These differences are small when $d$ is small.

In the next section we will show that $h_{*}\left(\mathcal{N}^{\prime}\right)=h^{*}\left(\mathcal{N}^{\prime}\right)$ and find explicitly its value. We also find asymptotics of number of different periodic solutions from $\mathcal{N}^{\prime}$ on a given interval $L$ as $L \rightarrow \infty$. According to (6.4) this gives estimates of the number of periodic solutions from $\mathcal{N}$ corresponding to $F$ with small $d$ and $d_{F}$.

Remark 6.1. The Bernoulli shift $l \rightarrow l+1$ determines dynamics of words from $\pi_{1}^{\infty}$ and we are able to find topological entropy which describes how fast complexity of

$$
b_{n}=\prod_{l=-n}^{n} g_{i_{l}}^{\kappa_{l}}, \quad \kappa_{l}= \pm 1, \quad i_{l}=1,2
$$

increases as $n \rightarrow \infty$. Since the number of elements equals $4 \times 3^{2 N}$, the topological entropy equals $\ln 3$ ( this was shown in [1] ). In this paper we apply a different approach. We study not only the topological structure of solutions but their spatial structure as well. We consider solutions on a spatial interval $[-L, L]$ and study how fast the complexity grows as $L \rightarrow \infty$.

\section{Suspended flow for minimizers of a special potential.}

In this section we consider curves $v=v_{b}$ introduced in the previous section. Two minimal cycles have centers at points $O_{1}, O_{2}, O_{1}=(-a, 0), O_{2}=(a, 0)$ in $u$-plane with the origin $O=(0,0)$. The radii $\eta_{0}$ of the minimal cycles are denoted by $R$. Generators of the fundamental group are still denoted by $g_{1}, g_{2}$. Note that since $F(u)=\mu$ in the definition of the Jacobian length of the restriction $\mathcal{J}\left(\left.v\right|_{S}\right)$ of $v(x)$ onto an interval $S=\left[x_{1}, x_{2}\right]$, we have the relation

$$
\mathcal{J}\left(\left.v\right|_{\left[x_{1}, x_{2}\right]}\right)=\sqrt{2 \mu} l_{E}\left(\left.v\right|_{\left[x_{1}, x_{2}\right]}\right)=2 \mu\left|x_{2}-x_{1}\right| .
$$

We put for simplicity $\mu=1 / 2$ and obtain results for $\mu \neq 1 / 2$ from Section 1 by a simple rescaling.

We consider the problem: how many closed geodesic curves of the considered type have the length (Euclidean) smaller than a number $L$, or between $L-1$ and $L$ ? In the course of solving this problem we also solve a simpler problem of estimation of the number of arbitrary curves (maybe not closed).

We need to recall a piece of the ergodic theory. Each closed geodesic corresponds to a irreducible word $g=g_{i_{1}}^{k_{1}} \ldots g_{i_{n}}^{k_{n}}$ where $k_{i} \in \mathbf{Z} \backslash\{0\}, i=$ $1, \ldots, n$ and $g_{i}$ is a generator of the fundamental group $\pi_{1}\left(D^{\prime}\right)$ where $D^{\prime}=$ 
$\bar{D}_{0} \backslash\left\{D_{1}^{1} \cup D_{2}^{1}\right\}$. Here $D_{1}^{1}, D_{2}^{1}$ are open disks of radius $R / 2$ centered at points $\mathrm{O}_{1}, \mathrm{O}_{2}$.

In order to describe all admissible words we introduced in [1] the following topological Markov chain. It has 4 states denoted by symbols 1,2 , 3,4 . We identified symbols 1,2 with generators $g_{1}, g_{2}$ and symbols 3,4 with elements $g_{1}^{-1}, g_{2}^{-1}$ correspondingly. We admitted a transition $i \rightarrow j$ if the pair $(i, j)$ does not correspond to the pair $g_{k} g_{k}^{-1}$ or $g_{k}^{-1} g_{k}, k=1,2$; $i, j=1,2,3,4$. So the matrix of transitions, say $A=\left(a_{i j}\right)_{i, j=1}^{4}$ is defined as follows: $a_{i j}=1$ if there exists a transition $i \rightarrow j$, and $a_{i j}=0$ if not, i.e.,

$$
a=\left(\begin{array}{llll}
1 & 1 & 0 & 1 \\
1 & 1 & 1 & 0 \\
0 & 1 & 1 & 1 \\
1 & 0 & 1 & 1
\end{array}\right) .
$$

The elements of $\pi_{1}^{\infty}$ given by (5.1) can be rewritten in new notations as follows. One can denote, as usual, by $\Omega_{4}$ the set of infinite sequences (words)

$$
\underline{\omega}=\left(\ldots \omega_{-1} \omega_{0} \ldots \omega_{k} \ldots\right), \quad \omega_{k} \in\{1,2,3,4\}
$$

with the following property: $a_{\omega, \omega_{k+1}}=1, k \in \mathbf{Z}$ (the last condition is equivalent to the irreducibility of the word). $\Omega_{4}$ is endowed with the metric

$$
\operatorname{dist}\left(\underline{\omega}^{\prime}, \underline{\omega}^{\prime \prime}\right)=\sum_{k=-\infty}^{\infty} \frac{1}{2^{|k|}}\left|\omega_{k}^{\prime}-\omega_{k}^{\prime \prime}\right|
$$

and is a compact Cantor-like metric space. The shift map $\tau: \Omega_{4} \rightarrow \Omega_{4}$ is defined by $(\tau \underline{\omega})_{k}=\omega_{k+1}$ if $(\underline{\omega})_{k}=\omega_{k}$; it is a homeomorphism. The dynamical system $\left(\tau^{n}, \Omega_{4}\right)$ is a topological Markov chain (or a subshift of finite type) determined by the matrix of transition $A$. By using the standard technique of the symbolic dynamics (see, for instance [2]) we showed in [1] that the homotopic complexity $\mathcal{K}\left(\mathcal{A}_{N}\right)$ of an attractor $\mathcal{A}_{N}$ which contains periodic solutions of all homotopic classes $g$ up to $\operatorname{deg} g \leq N$ is

$$
\mathcal{K}\left(\mathcal{A}_{N}\right)=\ln \left(2 N+\frac{1}{2}\left(3^{N+1}+(-1)^{N}-2\right)\right) .
$$

(Homotopic complexity is the log of the number of all words corresponding to closed curves $u(x)$ belonging to $\mathcal{A}_{N}$.)

Now we are going to answer the different question: How many homotopy different closed solutions have $x$-period $\leq L$ ? This problem is much more difficult; we consider the limit case with $d=0$ of the situation considered 


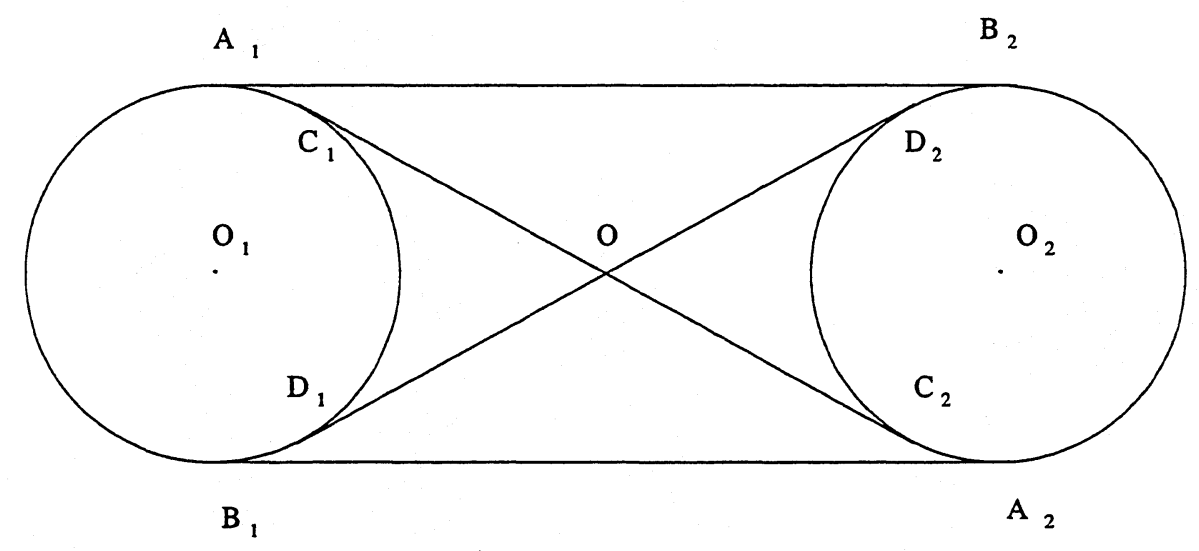

Figure 9.

in the previous section and we give here only asymptotics for a large $L$. According to the results of the previous section this gives estimates of $h_{*}$ and $h^{*}$ for solutions with $d>0$. (Recall that we consider solutions which satisfy (1.3) which gives the relation between the curve and its $x$-parametrization).

In other words we consider here a distribution of homotopically different closed curves $v_{b}$ along their length axis (but not along the "complexity axis" as in [1]).

The geodesic lines $v_{b}$ can be situated only along the following lines (see Fig. 9): Circles of radius $R$ centered at points $O_{1} O_{2}$, the interval $A_{1} B_{2}$ or $B_{1} A_{2}$ and the intervals $D_{1} D_{2}$ and $C_{1} C_{2}$ which are tangent to the circles.

For example the closed geodesic of minimal length which represents the word $g_{1} g_{2}^{-1}$ can be represented as $O C_{1} A_{1} B_{1} D_{1} O+O D_{2} B_{2} A_{2} C_{2} O$ or $A_{1} B_{1}+$ $B_{1} D_{1} O D_{2} B_{2}+B_{2} A_{2}+A_{2} C_{2} O C_{1} A_{1}$ and the word $g_{1} g_{2}$ is represented as $A_{1} B_{1}+B_{1} A_{2}+A_{2} B_{2}+B_{2} A_{1}$.

We give below an algorithm of the representation of a general closed geodesic as a union of standard pieces of lines.

Let $g=a_{1} a_{2} \ldots a_{n}$ be an irreducible word, $a_{k}=g_{1,2}^{ \pm 1}, k=1,2, \ldots, n$. Define the "adjusted" point $M_{k}$ as follows: $M_{k}:=A_{1}$ if $a_{k}=g_{1}, M_{k}:=B_{1}$ if $a_{k}=g_{1}^{-1}, M_{k}:=A_{2}$ if $a_{k}=g_{2}, M_{k}:=B_{2}$ if $a_{k}=g_{2}^{-1}$. Define now the "lines of increment" $M_{k} M_{k+1}, k=1, \ldots, n-1$ and $M_{n} M_{1}$ as shown in Table 1 (see Fig. 9). 


\begin{tabular}{|c|c|}
\hline & Table 1 \\
\hline$a_{k}, a_{k+1}$ & $M_{k} M_{k+1}$ \\
\hline$g_{1}, g_{1}$ & $A_{1} B_{1}+B_{1} D_{1} C_{1} A_{1}$ \\
\hline$g_{1}, g_{2}$ & $A_{1} B_{1}+B_{1} A_{2}$ \\
\hline$g_{1}, g_{2}^{-1}$ & $A_{1} B_{1}+B_{1} D_{1}+D_{1} O D_{2}+D_{2} B_{2}$ \\
\hline$g_{1}^{-1}, g_{1}^{-1}$ & $B_{1} A_{1}+A_{1} C_{1} D_{1} B_{1}$ \\
\hline$g_{1}^{-1}, g_{2}^{-1}$ & $B_{1} A_{1}+A_{1} B_{2}$ \\
\hline$g_{1}^{-1}, g_{2}$ & $B_{1} A_{1}+A_{1} C_{1}+C_{1} O C_{2}+C_{2} A_{2}$ \\
\hline$g_{2}, g_{2}$ & $A_{2} B_{2}+B_{2} D_{2} C_{2} A_{2}$ \\
\hline$g_{2}, g_{1}$ & $A_{2} B_{2}+B_{2} A_{1}$ \\
\hline$g_{2}, g_{1}^{-1}$ & $A_{2} B_{2}+B_{2} D_{2}+D_{2} O D_{1}+D_{1} B_{1}$ \\
\hline$g_{2}^{-1}, g_{2}^{-1}$ & $B_{2} A_{2}+A_{2} C_{2} D_{2} B_{2}$ \\
\hline$g_{2}^{-1}, g_{1}^{-1}$ & $B_{2} A_{2}+A_{2} B_{1}$ \\
\hline$g_{2}^{-1}, g_{1}$ & $B_{2} A_{2}+A_{2} C_{2}+C_{2} O C_{1}+C_{1} A_{1}$ \\
\hline
\end{tabular}

So, the length of the increment can take only three values: either $2 \pi R$, or $\pi R+2 a$, or $\pi R+2 \sqrt{a^{2}-R^{2}}+2 R \arcsin \frac{R}{a}$ where $a:=\left|A_{1} B_{2}\right| / 2, R \arcsin \frac{R}{a}=$ $\left|D_{2} B_{2}\right|$. Let $w:=2 \pi R, y:=\pi R+2 a, z:=\pi R+2 \sqrt{a^{2}-R^{2}}+2 R \arcsin \frac{R}{a}$.

Therefore the lengths of the increments can be represented in the form of the following table. 


\begin{tabular}{|c|c||c|c|}
\hline \multicolumn{4}{|c|}{ Table 2 } \\
\hline$a_{k}, a_{k+1}$ & $\widetilde{M_{k} M_{k+1}}$ & $a_{k}, a_{k+1}$ & $\widetilde{M_{k} M_{k+1}}$ \\
\hline 1,1 & $w$ & 3,3 & $w$ \\
\hline 1,2 & $y$ & 3,4 & $y$ \\
\hline 1,4 & $z$ & 3,2 & $z$ \\
\hline 2,2 & $w$ & 4,4 & $w$ \\
\hline 2,1 & $y$ & 4,3 & $y$ \\
\hline 2,3 & $z$ & 4,1 & $z$ \\
\hline
\end{tabular}

Let us recall that the symbols $1,2,3,4$ correspond to the elements $g_{1}, g_{2}, g_{1}^{-1}$, $g_{2}^{-1}$ respectively.

Thus, the length of the considered geodesic of the homotopic type $\left[\omega_{1}, \ldots, \omega_{k}\right]$ is the sum of the length of the corresponding increments. It allows us to determine this length as the period of a periodic orbit of a suspended flow going through the point $\underline{\omega}=\left(\ldots\left[\omega_{1} \ldots \omega_{k}\right]\left[\omega_{1} \ldots \omega_{k}\right] \ldots\right)$.

Recall the definition of the suspended flow of the symbolic dynamical system $(\tau, \Omega)$ where $\tau: \Omega \rightarrow \Omega$ is the shift map of a set of symbolic sequences. Let $\psi: \Omega \rightarrow \mathbf{R}$ be strictly positive. We define the suspension space (related to $\psi$ ) as

$$
\sum_{\psi}=\{(\underline{\omega}, t): \underline{\omega} \in \Omega, 0 \leq t \leq \psi(\underline{\omega})\}
$$

with the identification $(\underline{\omega}, \psi(\underline{\omega}))=(\tau \underline{\omega}, 0)$. The suspension flow $\tau_{\psi}$ (relative to $\psi)$ is defined as the "vertical" flow on $\sum_{\psi}$ given by $\tau_{\psi, t^{\prime}}(\underline{\omega}, t)=\left(\underline{\omega}, t+t^{\prime}\right)$ for $0 \leq t, t+t^{\prime} \leq \psi(\underline{\omega})$. In our case the function $\psi$ is a constant on each cylinder $\left[\omega_{0} \omega_{1}\right]$. For example (see Table 2) $\psi\left(\begin{array}{lllll}\ldots \omega_{-1} & 1 & 2 & \omega_{2} & \omega_{3} \ldots\end{array}\right)=y$ independent of $\omega_{-k}, k \geq 1$ and $\omega_{j}, j \geq 2$. In order to simplify the situation, we reduce this case to that when $\psi$ depends only on the coordinate $\omega_{0}$, i.e., $\psi(\underline{\omega})=\psi\left(\omega_{0}\right)$. For that, introduce the new topological Markov chain $\left(\tau, \Omega_{12}\right)$ with the 12 states which are the admissible pairs $(i, j)$ in the left columns of Table 2. The transition $(m, n) \rightarrow(p, q)$ exists iff $n=p$. (This procedure is well-known in the symbolic dynamics see, for instance [2].) The corresponding $12 \times 12$ matrix of transitions, say $B$, has now the following 
form

$\begin{array}{lllll}111 & 000 & 000 & 000 & w \\ 000 & 111 & 000 & 000 & y \\ 000 & 000 & 000 & 111 & z \\ 000 & 111 & 000 & 000 & w \\ 111 & 000 & 000 & 000 & y \\ 000 & 000 & 111 & 000 & z \\ 000 & 000 & 111 & 000 & w \\ 000 & 111 & 000 & 000 & z \\ 000 & 000 & 000 & 111 & y \\ 000 & 000 & 000 & 111 & w \\ 000 & 000 & 111 & 000 & y \\ 111 & 000 & 000 & 000 & z\end{array}$

The last column shows the values of the function $\psi(i, j)$ for the corresponding pair $(i, j)$. The pairs $(i, j)$ is ordered here in the same way as in the first column of Table 2 (from above, for example the 11th row corresponds to the pair $(4,3))$. It is well-known that the topological Markov chains $\left(\tau, \Omega_{4}\right)$ and $\left(\tau, \Omega_{12}\right)$ are topologically conjugate (in particular, they have the same number of periodic points of each period). We define the suspension space relative to $\psi$

$$
\sum_{\psi}^{12}=\left\{(\underline{\omega}, t) ; \underline{\omega} \in \Omega_{12}, 0 \leq t \leq \psi(\underline{\omega})\right\}
$$

and the corresponding suspension flow. The function $\psi$ depends only on $\omega_{0}: \psi(\underline{\omega})=\psi\left(\omega_{0}\right):=\psi_{\omega_{0}}$ and takes only the values $w, y, z$. We use the results of [24] to find the topological entropy of the suspension flow. It was proved in [24] that if there exists an integer $s$ such that $B^{s}$ consists of strictly positive elements (in our case this condition is satisfied) then there exists a unique unit vector $e=\left\{e_{i}\right\}$ with positive components and a number $\lambda>0$ for which

$$
\sum_{j=1}^{12} b_{i j} e_{j}=\lambda^{\psi_{i}} e_{i}, \quad i=1, \ldots 12 .
$$

In our case we can explicitly find this vector and this number.

Lemma 7.1. If $\lambda$ is the unique root of the equation

$$
\left(\frac{1}{\lambda}\right)^{w}+\left(\frac{1}{\lambda}\right)^{y}+\left(\frac{1}{\lambda}\right)^{z}=1
$$


and $e$ is the vector with the following components

$$
\begin{aligned}
& e_{1}=e_{4}=e_{7}=e_{10}=\frac{\lambda^{y+z}}{2 \sqrt{\lambda^{2(y+z)}+\lambda^{2(w+y)}+\lambda^{2(w+z)}}}, \\
& e_{2}=e_{5}=e_{9}=e_{11}=\frac{\lambda^{w+z}}{2 \sqrt{\lambda^{2(y+z)}+\lambda^{2(w+y)}+\lambda^{2(w+z)}}}, \\
& e_{3}=e_{6}=e_{8}=e_{12}=\frac{\lambda^{w+y}}{2 \sqrt{\lambda^{2(y+z)}+\lambda^{2(w+y)}+\lambda^{2(w+z)}}},
\end{aligned}
$$

then the equalities (7.1) are satisfied.

Proof. In our case the conditions (7.1) can be rewritten as

$$
\begin{aligned}
e_{1}+e_{2}+e_{3} & =\lambda^{w} e_{1}, & e_{4}+e_{5}+e_{6} & =\lambda^{y} e_{2}, \\
e_{10}+e_{11}+e_{12} & =\lambda^{z} e_{3}, & e_{4}+e_{5}+e_{6} & =\lambda^{w} e_{4}, \\
e_{1}+e_{2}+e_{3} & =\lambda^{y} e_{5}, & e_{7}+e_{8}+e_{9} & =\lambda^{z} e_{6}, \\
e_{7}+e_{8}+e_{9} & =\lambda^{w} e_{7}, & e_{4}+e_{5}+e_{6} & =\lambda^{z} e_{8}, \\
e_{10}+e_{11}+e_{12} & =\lambda^{y} e_{9}, & e_{10}+e_{11}+e_{12} & =\lambda^{w} e_{10} \\
e_{7}+e_{8}+e_{9} & =\lambda^{y} e_{11}, & e_{1}+e_{2}+e_{3} & =\lambda^{z} e_{12} .
\end{aligned}
$$

The direct substitution of the expressions (7.5) into these equations shows their validity provided the equation (7.2) is taken into account.

It was shown in [24] that a measure of maximal entropy of the suspension flow is obtained from the stationary Markov measure $\bar{m}$ in $\Omega_{12}$ for which the probability of the state $i$ is $e_{i}$ and the transition probabilities $p_{i j}$ are $p_{i j}=\frac{b_{i j} e_{j}}{\lambda^{4} e_{i}}$. It is simple to show that $p_{i j}$ has one of the values $0, \lambda^{-w}, \lambda^{-y}$ or $\lambda^{-z}$. More precisely,

$$
\begin{aligned}
\lambda^{-w} & =p_{1,1}=p_{2,4}=p_{3,10}=p_{4,4}=p_{5,1}=p_{6,7}=p_{7,7}=p_{8,4} \\
& =p_{9,10}=p_{10,10}=p_{11,7}=p_{12,1} \\
\lambda^{-y} & =p_{1,2}=p_{2,5}=p_{3,11}=p_{4,5}=p_{5,2}=p_{6,9}=p_{7,9}=p_{8,5} \\
& =p_{9,11}=p_{10,11}=p_{11,9}=p_{12,2} \\
\lambda^{-z} & =\text { nonzero others. }
\end{aligned}
$$

Lemma 7.2. The topological entropy $h_{0}$ of the suspension flow is $\ln \lambda$ where $\lambda$ is the unique root of the equation (7.2). 
Proof. The topological entropy coincides with the measure-theoretic (Kolmogorov-Sinai) entropy relative to the measure with maximal entropy for the suspension flow. In fact, it follows from [24] that it equals $\ln \lambda$. But we explain it in the following way. Thanks to the Abramov formula (see, for instance [25]) it is equal to

$$
h_{0}=\frac{h_{K S}(\tau, \bar{m})}{\int \psi d \bar{m}}
$$

where $h_{K S}(\tau, \bar{m})$ is the KS entropy of the shift $\tau$ relative to the measure $\bar{m}$. Let us calculate the integral first. Since $\psi$ is a constant on each cylinder then

$$
\begin{aligned}
& \int \psi d \bar{m}=\sum_{i=1}^{12} \psi_{i} e_{i} \\
& =\frac{2}{\sqrt{3} \sqrt{\lambda^{2(y+z)}+\lambda^{2(w+y)}+\lambda^{2(w+z)}}}\left(w \lambda^{y+z}+y \lambda^{w+z}+z \lambda^{w+y}\right) .
\end{aligned}
$$

Now the entropy of a Markov measure is given by

$$
h_{K S}(\tau, \bar{m})=-\sum_{i, j=1}^{12} e_{i} p_{i j} \log p_{i j}
$$

(see for instance [2]). By using equalities (7.6) we obtain

$$
\begin{array}{r}
h_{K S}(\tau, \bar{m})=-\lambda^{-w} \ln \lambda^{-w} \sum_{i=1}^{12} e_{i}-\lambda^{-y} \ln \lambda^{-y} \sum_{i=1}^{12} e_{i}-\lambda^{-z} \ln \lambda^{-z} \sum_{i=1}^{12} e_{i} \\
=\ln \lambda\left(w \lambda^{-w}+y \lambda^{-y}+z \lambda^{-z}\right) \frac{2}{\sqrt{3}} \frac{\left(\lambda^{y+z}+\lambda^{w+z}+\lambda^{w+y}\right)}{\sqrt{\lambda^{2(y+z)}+\lambda^{2(w+y)}+\lambda^{2(w+z)}}} .
\end{array}
$$

Therefore,

$$
\frac{h_{K S}(\tau, \bar{m})}{\int \psi d \bar{m}}=\frac{\ln \lambda\left(\lambda^{y+z}+\lambda^{w+z}+\lambda^{w+y}\right)\left(w \lambda^{-w}+y \lambda^{-y}+z y^{-z}\right)}{\left(w \lambda^{y+z}+y \lambda^{w+z}+z \lambda^{w+y}\right)} .
$$

Dividing the numerator and the denominator by $\lambda^{w+y+z}$ we obtain the expression

$$
\ln \lambda\left(\lambda^{-w}+\lambda^{-y}+\lambda^{-z}\right)
$$

which is equal to $\ln \lambda$, thanks to (7.2). 
Corollary 7.1. $h_{*}\left(\mathcal{N}^{\prime}\right)=h^{*}\left(\mathcal{N}^{\prime}\right)=\ln \lambda$.

Comparing with Proposition 6.1 where $n=2$ and using (7.2) we can find the average length $L_{\text {av }}$ of the homotopy element of solutions from $\mathcal{N}^{\prime}$ as the solution of

$$
3^{-w / L_{\mathrm{av}}}+3^{-y / L_{\mathrm{av}}}+3^{-z / L_{\mathrm{av}}}=1 .
$$

Let us recall the following results of [25] (in these results $L \rightarrow \infty$ ).

(i) The number $N(L)$ of periodic orbits of the suspension flow with periods smaller than $L$ satisfies the asymptotic relation

$$
N(L) \sim \frac{e^{h_{0} L}}{h_{0} L}
$$

where $h_{0}$ is the topological entropy of the suspension flow ([25], p. 109).

(ii) The number $M(L)$ of periodic orbits of the suspension flow with periods from $L$ to $L+1$ is asymptotically distributed as the probability density

$$
\frac{h_{0} e^{h_{0} L}}{e^{h_{0}}-1} \text {. }
$$

These results hold under so called weak-mixing conditions. These conditions are satisfied if the function $\psi(\omega)$ defined above and a constant are not cohomologous to each other. Let us recall that two functions $\psi, g$ are said to be cohomologous $(\psi \sim g)$ if there exists a continuous function $G$ such that

$$
\psi=g+G \circ \tau-G .
$$

If $g:=c$ is a constant then the equality is rewritten as

$$
\psi(\omega)=G(\tau(\omega))-G(\omega)+c, \quad \omega \in \Omega_{12} .
$$

Lemma 7.3. The function $\psi(\omega)$ is cohomologous to a constant if and only if $w=y=z$.

Proof. Assume that $\psi(\omega)$ is cohomologous to a constant c, i.e., (7.11) is satisfied. The point $\omega_{0}:=(\ldots 252525 \ldots)$ is 2-periodic (see matrix $B$ ). Therefore, $\psi\left(\underline{\omega_{0}}\right)=G\left(\tau \underline{\omega_{0}}\right)-G\left(\underline{\omega_{0}}\right)+c ; \tau^{2} \omega_{0}=\omega_{0}$, i.e., $\psi\left(\tau \underline{\omega_{0}}\right)=G\left(\underline{\omega_{0}}\right)-$ $G\left(\tau \underline{\omega_{0}}\right)+c$. Therefore, $\psi\left(\underline{\omega_{0}}\right)+\psi\left(\tau \underline{\omega_{0}}\right)=2 c$. But $\psi\left(\omega_{0}\right)=y=\psi\left(\bar{\tau} \omega_{0}\right)$. So $c=y$. The point $\omega_{1}:=(\ldots 1,3,12,1,3,12, \ldots)$ is 3 -periodic $\psi\left(\omega_{1}\right)=w$, $\psi\left(\tau \underline{\omega_{1}}\right)=z=\psi\left(\tau^{2} \underline{\omega_{1}}\right)$. So, $\psi\left(\underline{\omega_{1}}\right)+\psi\left(\tau \underline{\omega_{1}}\right)+\psi\left(\tau^{2} \underline{\omega_{1}}\right)=w+2 z=3 c$, 
i.e., $c=\frac{w+2 z}{3}$. The point $\underline{\omega}_{2}=(\ldots 1,2,5,1,2,5, \ldots)$ is 3 -periodic and $\psi\left(\underline{\omega_{2}}\right)=w, \psi\left(\tau \underline{\omega_{2}}\right)=y=\psi\left(\tau^{2} \underline{\omega_{2}}\right)$. Thus, $w+2 y=3 c, c=\frac{w+2 y}{3}$. The equalities $c=y=\frac{w+2 z}{3}=\frac{w+2 y}{3}$ imply $w=y=z$.

By definition, $w, y$ and $z$ cannot be equal to the same quantity, thus the suspended flow possesses the weak-mixing property for every considered value of $w, y$ and $z$. Obviously, the length of periodic curves $v_{b}$ and the periods of the corresponding periodic orbits in the suspended flow coincide. Therefore, results of [25], and Lemmas above imply the following main statement of this section.

Theorem 7.1. The lengths of periodic curves $v_{b}$ are distributed in the following way:

(i) the number of these curves with the length smaller than $L$, which we denote by $\tilde{N}(L)$, satisfies

$$
\tilde{N}(L) \sim \lambda^{L}
$$

(ii) The number of these curves with lengths from $L$ to $L+1$, which we denote by $\widetilde{M}(L)$, is asymptotically distributed as

$$
\frac{\lambda^{L} \cdot \ln \lambda}{\lambda-1}
$$

where $\lambda$ is a unique root of the equation (7.2): $\lambda^{-w}+\lambda^{-y}+\lambda^{-z}=1$ and $w=2 \pi R, y=\pi R+2 a, z=\pi R+2 \sqrt{a^{2}-R^{2}}+2 R \arcsin \frac{R}{a}$ are the parameters of the problem.

Let us consider different two limit cases.

Remark 7.1. Let $R$ go to 0 . It is natural to assume that $\tilde{N}(L)$ has to increase since rotations around circles of radius $R$ almost do not influence the energy. It is really so. As $R \rightarrow 0$ then $w \simeq 2 \pi R, y \simeq 2 a \simeq z$ and we can derive that $R \simeq \frac{1}{\pi \lambda^{2 a} \ln \lambda}$ or $\left(\frac{1}{\pi R}\right)^{1 /(2 a+1)}<\lambda<\left(\frac{1}{\pi R}\right)^{1 /(2 a)}, R \rightarrow 0$, i.e., $\tilde{N}(L)$ grows "polynomially," not faster than $R^{-L /(2 a)}$ and not slower than $R^{-L /(2 a+1)}$. The expression (7.12) behaves asymptotically as $-R^{-\frac{L-1}{2 a}} \ln R$ or $-R^{-\frac{L-1}{2 a+1}} \ln R$. 
Remark 7.2. If $a \rightarrow \infty$ then quantities $\widetilde{N}(L)$ and $\widetilde{M}(L)$ have to, of course, decrease. In this case $w=2 \pi R, y \simeq 2 a+\pi R \simeq z, a \rightarrow \infty$. Asymptotically for the solution of (7.2) we have

$$
\lambda=1+\gamma, \gamma \simeq \frac{1}{2 a} \ln \frac{a}{\pi R} .
$$

\section{Acknowledgments.}

The authors want to express gratitude to Jack Hale and Jarek Kwapisz for useful discussions; they also want to thank the anonymous reviewer for numerous valuable remarks. A. B. and V. A. thank CDSNS for support during work on this paper. Work of A. B. was partially supported by INTAS grant 96-1158.

\section{References.}

[1] V.S. Afraimovich, A.V. Babin, and S.N. Chow, Spatial Chaotic Structure of attractors of reaction-diffusion systems, Transactions Amer. Math. Soc. 348 (1996), 5031-5063.

[2] V.M. Alekseyev, Symbolic Dynamics, 11th Summer Mathematical School, Mathematics Institute of Ukrainian Academy of Sciences Kiev, 1976.

[3] D.V. Anosov, Geodesic flows on closed Riemannian manifolds with negative curvature, Proc. Steklov. Inst. Mat. 90 (1967), 1-235.

[4] A.V. Babin, Dynamics of spatially chaotic solutions of parabolic equations, Matem.Sbornik 186:10 (1995), 1389-1415 (Engl transl. p. 3-30) (1995)

[5] A.V. Babin and L. Bunimovich, Dynamics of stable chaotic waves generated by hyperbolic PDE, Nonlinearity, 9 (1996), 853-875.

[6] A.V.Babin and M.I.Vishik, Attractors of Evolution equations, North-Holland, 1992.

[7] A.V.Babin and M.I.Vishik, Attractors of partial differential evolution equations in an unbounded domain, Proc. Roy. Soc. Edinburgh, 116A (1990), 221-243.

[8] V. Bangert, Mather sets for twist maps and geodesics on tori, Dynamics reported, vol. 1 (eds. U. Kirchgraber and H.O. Walter), John Wiley, 1988.

[9] M.L. Bialy and L.V. Polterovich, Geodesic flows on the two-dimensional torus and "commensurability-incommensurability" phase transitions, Funktsional. Anal. i Prilozhen. 20 (1986), 9-16. 
[10] S.V. Bolotin and P.H. Rabinowitz, A variational construction of chaotic trajectories for a reversible Hamiltonian system, J. Differential Equations 148 (1998), 364-387.

[11] S.V. Bolotin and P.H. Rabinowitz, A variational construction of chaotic trajectories for a Hamiltonian system on a torus, Boll. Un. Mat. Ital., to appear.

[12] A.T. Fomenko, D.G. Fuchs, and V.V. Gutenmacher, Homotopic Topology, Academiai Kiade, Budapest, 1986.

[13] J.K.Hale, Asymptotic Behavior of Dissipative Systems, Math Surveys and Monographs, Vol. 25, Amer. Math. Soc., Providence, RI, 1988.

[14] G.A. Hedlund, Geodesics on a two-dimensional Riemannian manifold with periodic coefficients, Ann. of Math. 33 (1932), 719-739.

[15] P. Eberlein, Geodesic flow in certain manifolds without conjugate points, Trans. Amer. Math. Soc. 167 (1972), 151-270.

[16] V.V. Kozlov, Calculus of variations in the large and classical mechanics, Uspekhi Mat. Nauk 40 (1985), 33-60.

[17] N.V.Krylov, Nonlinear elliptic and parabolic equations of second order, Reidel, 1987.

[18] A. Katok and B. Hassenblatt, Introduction to the Modern Theory of Dynamical Systems, Cambridge University Press, 1995.

[19] O.A. Ladyzenskaja, V.A. Solonnikov, and N.N. Ural'seva, Linear and quasilinear equations of parabolic type, Amer. Math. Soc., Providence, 1968.

[20] W.S. Massey, Algebraic Topology: An Introduction, Harcourt, Brace and World, Inc., New York, 1967.

[21] M. Morse, A fundamental class of geodesics on any closed surface of genus greater than one, Trans. Am. Math. Soc. 26 (1924), 25-60.

[22] J. Moser, Minimal solutions of variational problems on a torus, Ann. Inst. Henry Poincaré - Analyse non linéaire, 3 (1986), 229-272.

[23] J. Mather, Variational construction of orbits of twist diffeomorphisms, Jour. of A.M.S. 4 (1991), 207-263.

[24] Ya.G. Sinai, Gibbs measures in ergodic theory, Russ. Math. Surv. 27 (1972), 21-70.

[25] W. Parry and M. Pollicott, Zeta function and the periodic orbit structure of hyperbolic dynamics, Astèrisque, 268 (1990), 187-188. 
[26] G.W. Whitehead, Elements of Homotopy Topology, Springer-Verlag, 1978.

IICO-UASLP,

SAN LUIS POTOSI 78000 ,

SLP, MEXICO

UNIVERSITY OF CALIFORNIA, IRVINE CA 92697-3875, U.S.A.

AND

CDSNS and School of Mathematics, Georgia Institute of TeChNology, AtLANTA, GA 30332-0160, U.S.A.

ReCeived February 24, 1999. 